\title{
WELL-POSEDNESS OF THE PRANDTL EQUATION IN SOBOLEV SPACES
}

\author{
R. ALEXANDRE, Y.-G. WANG, C.-J. XU, AND T. YANG
}

\section{INTRODUCTION}

In this work, we study the well-posedness of the Prandtl equation which is the foundation of the boundary layer theory introduced by Prandtl in 1904 [20]. It describes the behavior of the flow near a boundary in the small viscosity limit of an incompressible viscous flow with the non-slip boundary condition. We consider the following initial-boundary value problem:

$$
\left\{\begin{array}{l}
u_{t}+u u_{x}+v u_{y}+p_{x}=u_{y y}, \quad t>0, \quad x \in \mathbb{R}, \quad y>0, \\
u_{x}+v_{y}=0, \\
\left.u\right|_{y=0}=\left.v\right|_{y=0}=0, \quad \lim _{y \rightarrow+\infty} u=U(t, x), \\
\left.u\right|_{t=0}=u_{0}(x, y),
\end{array}\right.
$$

where $u(t, x, y)$ and $v(t, x, y)$ represent the tangential and normal velocities of the boundary layer, with $y$ being the scaled normal variable to the boundary, while $U(t, x)$ and $p(t, x)$ are the values on the boundary of the tangential velocity and pressure of the outflow satisfying the Bernoulli law

$$
\partial_{t} U+U \partial_{x} U+\partial_{x} p=0 .
$$

The well-posedness theories and the justification of the Prandtl equation remain as the challenging problems in the mathematical theory of fluid mechanics. Up to now, there are only a few rigorous mathematical results. Under a monotonic assumption on the tangential velocity of the outflow, Oleinik was the first to obtain the local existence of classical solutions for the initial-boundary value problems in [18], and this result together with some of her works with collaborators were well presented in the monograph [19]. In addition to Oleinik's monotonicity assumption on the velocity field, by imposing a so called favorable condition on the pressure, Xin and Zhang obtained the existence of global weak solutions to the Prandtl equation in [22]. All these well-posedness results were based on the Crocco transformation to overcome the main difficulty caused by degeneracy and mixed type of the equation.

Without the monotonicity assumption, E and Engquist in [5] constructed some finite time blowup solutions to the Prandtl equation. And in [21], Sammartino and Caflisch obtained the local existence of analytic solutions to the Prandtl equation, and a rigorous theory on the stability of boundary layers with analytic data in the

Received by the editors March 7, 2012 and, in revised form, April 10, 2014.

2010 Mathematics Subject Classification. Primary 35M13, 35Q35, 76D10, 76D03, 76N20.

Key words and phrases. Prandtl equation, well-posedness theory, Sobolev spaces, energy method, monotonic velocity field, Nash-Moser-Hörmander iteration. 
framework of the abstract Cauchy-Kowaleskaya theory. This result was extended to the function space which is only analytic in the tangential variable in [14.

In recent years, there have been some interesting works concerning the linear and nonlinear instability of the Prandtl equation in the Sobolev spaces. In [8, Grenier showed that the unstable Euler shear flow $\left(u_{s}(y), 0\right)$ with $u_{s}(y)$ having an inflection point (the well-known Rayleigh's criterion) yields instability for the Prandtl equation. In the spirit of Grenier's approach, in [6], Gérard-Varet and Dormy showed that if the shear flow profile $\left(u^{s}(t, y), 0\right)$ of the Prandtl equation has a non-degenerate critical point, then it leads to a strong linear ill-posedness of the Prandtl equation in the Sobolev framework. In a similar approach, in [7] Gérard-Varet and Nguyen strengthened the ill-posedness result of [6] for the linearized Prandtl equation for an unstable shear flow. Moreover, they also showed that if a solution, as a small perturbation of the unstable shear flow, to the nonlinear Prandtl equation exists in the Sobolev setting, then it cannot be Lipschitz continuous. Along this direction, Guo and Nguyen in [9] proved that the nonlinear Prandtl equation is ill-posed near non-stationary and non-monotonic shear flows, and showed that the asymptotic boundary-layer expansion is not valid for nonmonotonic shear layer flows in Sobolev spaces. Hong and Hunter [11] studied the formation of singularities and instability in the unsteady inviscid Prandtl equation. All these works show what happens in an unsteady boundary layer separation. For the related mathematical results and discussions, also see the review papers [2,4.

As mentioned in [2,6,7, the local in time well-posedness of the Prandtl equation for initial data in Sobolev space remains an open problem. Therefore, it would be very interesting to recover Oleinik's well-posedness results simply through direct energy estimates. And this is the goal of this paper.

In this paper we consider the case of a uniform outflow $U=1$, which implies $p$ being a constant. Consider the following problem for the Prandtl equation:

$$
\left\{\begin{array}{l}
u_{t}+u u_{x}+v u_{y}-u_{y y}=0, \quad t>0, \quad x \in \mathbb{R}, \quad y>0, \\
u_{x}+v_{y}=0, \\
\left.u\right|_{y=0}=\left.v\right|_{y=0}=0, \quad \lim _{y \rightarrow+\infty} u=1 \\
\left.u\right|_{t=0}=u_{0}(x, y) .
\end{array}\right.
$$

We are going to study the well-posedness of the Prandtl equation around a monotonic shear flow. More precisely, assume that

$$
u_{0}(x, y)=u_{0}^{s}(y)+\tilde{u}_{0}(x, y),
$$

where $u_{0}^{s}$ is monotonic in $y$,

$$
\partial_{y} u_{0}^{s}(y)>0, \quad \forall y \geq 0 .
$$

To state the main result, we first introduce the following notations. Set $\Omega_{T}=$ $[0, T] \times \mathbb{R}_{+}^{2}$. For any non-negative integer $k$ and real number $\ell$, define

$$
\|u\|_{\mathcal{A}_{\ell}^{k}\left(\Omega_{T}\right)}=\left(\sum_{k_{1}+\left[\frac{k_{2}+1}{2}\right] \leq k}\left\|\langle y\rangle^{\ell} \partial_{(t, x)}^{k_{1}} \partial_{y}^{k_{2}} u\right\|_{L^{2}\left([0, T] \times \mathbb{R}_{+}^{2}\right)}^{2}\right)^{1 / 2},
$$

and

$$
\|u\|_{\mathcal{D}_{\ell}^{k}\left(\Omega_{T}\right)}=\sum_{k_{1}+\left[\frac{k_{2}+1}{2}\right] \leq k}\left\|\langle y\rangle^{\ell} \partial_{(t, x)}^{k_{1}} \partial_{y}^{k_{2}} u\right\|_{L_{y}^{\infty}\left(L_{t, x}^{2}\right)},
$$


with $\langle y\rangle=\left(1+y^{2}\right)^{\frac{1}{2}}$. When the function is independent of the $t$ (or $x$ ) variable, we use the same notations for the non-isotropic norms as above under the convention that we do not take integration with respect to this variable.

The main result of this paper can be stated as follows.

Theorem 1.1. Concerning the problem (1.2), we have the following existence and stability results.

(1) Given any integer $k \geq 5$ and real number $\ell>\frac{1}{2}$, let the initial data $u_{0}(x, y)=$ $u_{0}^{s}(y)+\tilde{u}_{0}(x, y)$ satisfy the compatibility conditions of the initial boundary value problem (1.2) up to order $k+4$. Assume the following two conditions:

(i) The monotonicity condition (1.3) holds for $u_{0}^{s}$, and

$$
\left\{\begin{array}{l}
\partial_{y}^{2 j} u_{0}^{s}(0)=0, \quad \forall 0 \leq j \leq k+4, \\
\left\|\langle y\rangle^{\ell}\left(u_{0}^{s}-1\right)\right\|_{H^{2 k+9}\left(\mathbb{R}_{+}^{2}\right)}+\left\|\frac{\partial_{y}^{2} u_{0}^{s}}{\partial_{y} u_{0}^{s}}\right\|_{H^{2 k+7}\left(\mathbb{R}_{+}^{2}\right)} \leq C,
\end{array}\right.
$$

for a fixed constant $C>0$.

(ii) There exists a small constant $\epsilon>0$ depending only on $u_{0}^{s}$, such that

$$
\left\|\tilde{u}_{0}\right\|_{\mathcal{A}_{\ell}^{2 k+9}\left(\mathbb{R}_{+}^{2}\right)}+\left\|\frac{\partial_{y} \tilde{u}_{0}}{\partial_{y} u_{0}^{s}}\right\|_{\mathcal{A}_{\ell}^{2 k+9}\left(\mathbb{R}_{+}^{2}\right)} \leq \epsilon .
$$

Then there is $T>0$, such that the problem (1.2) admits a classical solution $u$ satisfying

$$
u-u^{s} \in \mathcal{A}_{\ell}^{k}\left(\Omega_{T}\right), \frac{\partial_{y}\left(u-u^{s}\right)}{\partial_{y} u_{0}^{s}} \in \mathcal{A}_{\ell}^{k}\left(\Omega_{T}\right), v \in \mathcal{D}_{0}^{k-1}\left(\Omega_{T}\right) .
$$

Here, note that $\left(u^{s}(t, y), 0\right)$ is the shear flow of the Prandtl equation defined by the initial data $\left(u_{0}^{s}(y), 0\right)$.

(2) The solution is unique in the function space described in (1.6). Moreover, we have the stability with respect to the initial data in the following sense. For any given two initial data

$$
u_{0}^{1}=u_{0}^{s}+\tilde{u}_{0}^{1}, \quad u_{0}^{2}=u_{0}^{s}+\tilde{u}_{0}^{2},
$$

if $u_{0}^{s}$ satisfy (1.3), (1.4), and $\tilde{u}_{0}^{1}, \tilde{u}_{0}^{2}$ satisfy (1.5), then the corresponding solutions $\left(u^{1}, v^{1}\right)$ and $\left(u^{2}, v^{2}\right)$ of (1.2) satisfy

$$
\left\|u^{1}-u^{2}\right\|_{\mathcal{A}_{\ell}^{p}\left(\Omega_{T}\right)}+\left\|v^{1}-v^{2}\right\|_{\mathcal{D}_{0}^{p-1}\left(\Omega_{T}\right)} \leq C\left\|\frac{\partial}{\partial y}\left(\frac{u_{0}^{1}-u_{0}^{2}}{\partial_{y} u_{0}^{s}}\right)\right\|_{\mathcal{A}_{\ell}^{p}\left(\mathbb{R}_{+}^{2}\right)},
$$

for all $p \leq k-1$, where the constant $C>0$ depends only on $T$ and the upper bounds of the norms of $u_{0}^{1}, u_{0}^{2}$.

Remark 1.2. Note that the solutions obtained in the above theorem are less regular than the initial data mainly due to the degeneracy with respect to the tangential variable $x$ of the Prandtl equation.

Remark 1.3. (1) It is not difficult to see from the proof of Theorem 1.1 that the above main result holds also for the problem (1.2) defined in the torus $\mathbb{T}^{1}$ for the $x$-variable.

(2) Note that our approach for the above well-posedness result can be generalized to study the nonlinear problem (1.1) with a non-trivial Euler outflow. Unlike the case with a uniform outflow, in general we do not have an explicit background state like the shear flow $\left(u^{s}(t, y), 0\right)$. Therefore, the main difference is how to construct 
a proper zeroth order approximation for the iteration scheme (4.9) and (4.10), so that the error in the equations is small and sufficiently fast decay in $y$ at infinity. This can be done as follows. First, one constructs an approximation by the Taylor expansion in through the compatibility condition of the initial data and the equations. Then, by studying the linearized problem around this approximation, one can reach a proper approximation satisfying the original nonlinear problems with a small and fast decay error, under a suitable convergence rate of the initial data to the outflow.

The rest of the paper is organized as follows. In Section 2, we will introduce some weighted non-isotropic Sobolev spaces which will be used later, and we give the properties of the monotonic shear flow produced by the initial data $\left(u_{0}^{s}(y), 0\right)$. Then, in Section 3 , we will study the well-posedness of the linearized problem of the Prandtl equation (1.2) in the Sobolev spaces by a direct energy method, when the background tangential velocity is monotonic in the normal variable. Again, note that without using the Crocco transformation, the approach introduced here is completely new and will have further applications. The proof of Theorem 1.1 will be presented in Section 4. Section 5, and Section 6. As we mentioned earlier, from the energy estimates obtained in Section 3 for the linearized Prandtl equation, there is a loss of regularity of solutions with respect to the background state and the initial data. For this, we apply the Nash-Moser-Hörmander iteration scheme to study the nonlinear Prandtl equation. In Section 4, we will first construct the iteration scheme for the problem (1.2), then in Section 5 we will prove the convergence of this iteration scheme by a series of estimates, and then we will conclude with the existence of solutions to the Prandtl equation. The uniqueness and stability of the solution will be proved in Section [6. Finally, Section 7 is devoted to the proof of several technical estimates used in Section 5 .

\section{Preliminary}

As a preparation, in this section we introduce some function spaces which will be used later. And the properties of a monotonic shear flow will also be given.

2.1. Weighted non-isotropic Sobolev spaces. Since the Prandtl equation given in (1.2) is a degenerate parabolic equation coupled with the divergence-free condition, it is natural to work in some weighted anisotropic Sobolev spaces.

In addition to the spaces $\mathcal{A}_{\ell}^{k}$ and $\mathcal{D}_{\ell}^{k}$ introduced already in Section 1 we also need the following function spaces.

Denote by $\partial_{\mathcal{T}}^{k}$ the summation of tangential derivatives $\partial_{\mathcal{T}}^{\beta}=\partial_{t}^{\beta_{0}} \partial_{x}^{\beta_{1}}$ for all $\beta=$ $\left(\beta_{0}, \beta_{1}\right) \in \mathbb{N}^{2}$ with $|\beta| \leq k$. Recall $\langle y\rangle=\left(1+|y|^{2}\right)^{1 / 2}$. For any given $k, k_{1}, k_{2} \in$ $\mathbb{N}, \lambda \geq 0, \ell \in \mathbb{R}$ and $0<T<+\infty$, we introduce

$$
\begin{aligned}
& \|f\|_{\mathcal{B}_{\lambda, \ell}^{k_{1}, k_{2}}}=\left(\sum_{0 \leq m \leq k_{1}, 0 \leq q \leq k_{2}}\left\|e^{-\lambda t}\langle y\rangle^{\ell} \partial_{\mathcal{T}}^{m} \partial_{y}^{q} f\right\|_{L^{2}\left([0, T] \times \mathbb{R}_{+}^{2}\right)}^{2}\right)^{1 / 2}, \\
& \|f\|_{\tilde{\mathcal{B}}_{\lambda, \ell}^{k_{1}, k_{2}}}=\left(\sum_{0 \leq m \leq k_{1}, 0 \leq q \leq k_{2}}\left\|e^{-\lambda t}\langle y\rangle^{\ell} \partial_{\mathcal{T}}^{m} \partial_{y}^{q} f\right\|_{L^{\infty}\left([0, T] ; L^{2}\left(\mathbb{R}_{+}^{2}\right)\right)}^{2}\right)^{1 / 2},
\end{aligned}
$$


and

$$
\|u\|_{\mathcal{C}_{\ell}^{k}}=\sum_{k_{1}+\left[\frac{k_{2}+1}{2}\right] \leq k}\left\|\langle y\rangle^{\ell} \partial_{\mathcal{T}}^{k_{1}} \partial_{y}^{k_{2}} u\right\|_{L_{y}^{2}\left(L_{t, x}^{\infty}\right)} .
$$

For the space $\mathcal{A}_{\ell}^{k}$ introduced in Section 1, obviously, we have

$$
\mathcal{A}_{\ell}^{k}=\bigcap_{j=0}^{k} \mathcal{B}_{0, \ell}^{k-j, 2 j} .
$$

As mentioned in Section [1] when the function is independent of the $t$ (or $x$ ) variable, we use the same notations for the non-isotropic norms defined above under the convention that we do not take integration or supremum with respect to this variable. Note that the parameter $\lambda$ is associated to the variable $t$ and the parameter $\ell$ to the variable $y$.

The homogeneous norms $\|\cdot\|_{\dot{\mathcal{A}}_{\ell}^{k}},\|\cdot\|_{\dot{\mathcal{C}}_{\ell}^{k}},\|\cdot\|_{\dot{\mathcal{D}}_{\ell}^{k}}$ correspond to the summation $1 \leq k_{1}+\left[\frac{k_{2}+1}{2}\right] \leq k$ in the definitions.

For $1 \leq p \leq+\infty$, we will also use $\|f\|_{L_{\ell}^{p}\left(\mathbb{R}_{+}^{2}\right)}=\left\|\langle y\rangle^{\ell} f\right\|_{L^{p}\left(\mathbb{R}_{+}^{2}\right)}$.

By classical theory, it is easy to get the following Sobolev-type embeddings:

$$
\|u\|_{\mathcal{C}_{\ell}^{k}} \leq C_{s}\|u\|_{\mathcal{A}_{\ell}^{k+2}}, \quad\|u\|_{\mathcal{D}_{\ell}^{k}} \leq C_{s}\|u\|_{\mathcal{A}_{\ell}^{k+1}} .
$$

Moreover, for any $\ell \geq 0$ and $k \geq 2$, the space $\mathcal{A}_{\ell}^{k}$ is continuously embedded into $C_{b}^{k-2}$, the space of $(k-2)$-th order continuously differentiable functions with all derivatives being bounded.

In the following, we will use some Morse-type inequalities for the above four function spaces, which are consequences of interpolation inequality and the fact that the space $L^{2} \cap L^{\infty}$ is an algebra.

Lemma 2.1. For any proper functions $f$ and $g$, we have

$$
\|f g\|_{\mathcal{A}_{\ell}^{k}} \leq M_{k}\left\{\|f\|_{\mathcal{A}_{\ell}^{k}}\|g\|_{L^{\infty}}+\|f\|_{L^{\infty}}\|g\|_{\dot{\mathcal{A}}_{\ell}^{k}}\right\}
$$

and

$$
\|f g\|_{\mathcal{A}_{\ell}^{k}} \leq M_{k}\left\{\|f\|_{\mathcal{C}_{\ell}^{k}}\|g\|_{\mathcal{D}_{0}^{0}}+\|f\|_{\mathcal{C}_{\ell}^{0}}\|g\|_{\dot{\mathcal{D}}_{0}^{k}}\right\} .
$$

Similar inequalities hold for the norms $\|\cdot\|_{\mathcal{B}_{\ell}^{k_{1}, k_{2}}},\|\cdot\|_{\mathcal{C}_{\ell}^{k}}$ and $\|\cdot\|_{\mathcal{D}_{\ell}^{k}}$. Here, $M_{k}>0$ is a constant depending only on $k$.

This result can be obtained in a way similar to that given in [15].

2.2. Properties of a monotonic shear flow. Let $u^{s}(t, y)$ be the solution of the following initial boundary value problem:

$$
\left\{\begin{array}{l}
\partial_{t} u^{s}=\partial_{y}^{2} u^{s}, \quad t>0, \quad y>0, \\
\left.u^{s}\right|_{y=0}=0, \quad \lim _{y \rightarrow+\infty} u^{s}(t, y)=1, \quad t>0, \\
\left.u^{s}\right|_{t=0}=u_{0}^{s}(y), \quad y>0 .
\end{array}\right.
$$

Note that $\left(u^{s}(t, y), 0\right)$ is a shear flow for the Prandtl equation (1.2).

Proposition 2.2. For any fixed $k \geq 2$, assume that the initial data $u_{0}^{s}(y)$ satisfy the monotonicity condition

$$
\partial_{y} u_{0}^{s}(y)>0, \quad \forall y \geq 0,
$$


and the compatibility conditions for (2.2) up to order $k$, i.e.,

$$
\lim _{y \rightarrow+\infty} u_{0}^{s}(y)=1, \quad \partial_{y}^{2 j} u_{0}^{s}(0)=0, \quad \forall 0 \leq j \leq k .
$$

Moreover, assume that

$$
\left\|u_{0}^{s}-1\right\|_{L^{2}\left(\mathbb{R}_{+}\right)}+\left\|u_{0}^{s}\right\|_{L^{\infty}\left(\mathbb{R}_{+}\right)}+\left\|u_{0}^{s}\right\|_{\dot{\mathcal{C}}_{\ell}^{k}}+\left\|\frac{\partial_{y}^{2} u_{0}^{s}}{\partial_{y} u_{0}^{s}}\right\|_{\mathcal{C}_{0}^{k-1}} \leq C,
$$

for a constant $C>0$ and a fixed $\ell>0$. Then, we have

$$
\partial_{y} u^{s}(t, y)>0, \quad \forall t, y \geq 0,
$$

and for any fixed $T>0$, there is a constant $C(T)$ such that

$$
\left\|u^{s}\right\|_{L^{\infty}\left([0, T] \times \mathbb{R}_{+}\right)}+\left\|u^{s}\right\|_{\mathcal{C}_{\ell}^{k}}+\left\|\frac{\partial_{y}^{2} u^{s}}{\partial_{y} u^{s}}\right\|_{\mathcal{C}_{0}^{k-1}} \leq C(T) .
$$

Moreover, for a fixed $0<R_{0}<T$, there is a constant $C\left(T, R_{0}\right)>0$ such that

$$
\max _{0 \leq \bar{y} \leq R_{0}}\left\|\frac{\partial_{y} u^{s}(t, y+\bar{y})}{\partial_{y} u^{s}(t, y)}\right\|_{\mathcal{C}_{0}^{k-1}\left([0, T] \times \mathbb{R}_{y}^{+}\right)} \leq C\left(T, R_{0}\right)
$$

and

$$
\max _{0 \leq \bar{t} \leq R_{0}}\left\|\frac{\partial_{y} u^{s}(t+\bar{t}, y)}{\partial_{y} u^{s}(t, y)}\right\|_{\mathcal{C}_{0}^{k-2}\left(\left[0, T-R_{0}\right] \times \mathbb{R}_{y}^{+}\right)} \leq C\left(T, R_{0}\right) .
$$

Proof. Obviously, the solution of (2.2) can be written as

$$
\begin{aligned}
u^{s}(t, y) & =\frac{1}{2 \sqrt{\pi t}} \int_{0}^{+\infty}\left(e^{-\frac{(y-\tilde{y})^{2}}{4 t}}-e^{-\frac{(y+\tilde{y})^{2}}{4 t}}\right) u_{0}^{s}(\tilde{y}) d \tilde{y} \\
& =\frac{1}{\sqrt{\pi}}\left(\int_{-\frac{y}{2 \sqrt{t}}}^{+\infty} e^{-\xi^{2}} u_{0}^{s}(2 \sqrt{t} \xi+y) d \xi-\int_{\frac{y}{2 \sqrt{t}}}^{+\infty} e^{-\xi^{2}} u_{0}^{s}(2 \sqrt{t} \xi-y) d \xi\right),
\end{aligned}
$$

which gives

$$
\begin{aligned}
\partial_{t} u^{s}(t, y)= & \frac{1}{\sqrt{\pi t}}\left(\int_{-\frac{y}{2 \sqrt{t}}}^{+\infty} \xi e^{-\xi^{2}}\left(\partial_{y} u_{0}^{s}\right)(2 \sqrt{t} \xi+y) d \xi\right. \\
& \left.-\int_{\frac{y}{2 \sqrt{t}}}^{+\infty} \xi e^{-\xi^{2}}\left(\partial_{y} u_{0}^{s}\right)(2 \sqrt{t} \xi-y) d \xi\right) .
\end{aligned}
$$

By using $\partial_{y}^{2 j} u_{0}^{s}(0)=0$ for $0 \leq j \leq k$, it follows that

$$
\begin{aligned}
\partial_{y}^{p} u^{s}(t, y)= & \frac{1}{\sqrt{\pi}}\left(\int_{-\frac{y}{2 \sqrt{t}}}^{+\infty} e^{-\xi^{2}}\left(\partial_{y}^{p} u_{0}^{s}\right)(2 \sqrt{t} \xi+y) d \xi\right. \\
& \left.+(-1)^{p+1} \int_{\frac{y}{2 \sqrt{t}}}^{+\infty} e^{-\xi^{2}}\left(\partial_{y}^{p} u_{0}^{s}\right)(2 \sqrt{t} \xi-y) d \xi\right)
\end{aligned}
$$

for all $1 \leq p \leq 2 k$, from which one immediately deduces the monotonicity property (2.4).

To estimate the last term given in (2.5), denote

$$
\alpha(t, y)=\frac{\partial_{y}^{2} u^{s}}{\partial_{y} u^{s}}(t, y)
$$


Then, from (2.2), we know that $\alpha(t, y)$ satisfies the following initial boundary value problem for the Burgers equation:

$$
\left\{\begin{array}{l}
\partial_{t} \alpha=\partial_{y}^{2} \alpha+2 \alpha \partial_{y} \alpha, \\
\left.\alpha\right|_{y=0}=0, \quad t>0, \\
\left.\alpha\right|_{t=0}=\alpha^{0}(y):=\frac{\partial_{y}^{2} u_{0}^{s}}{\partial_{y} u_{0}^{s}}(y), \quad y>0 .
\end{array}\right.
$$

It is easy to verify that the compatibility conditions of (2.8) hold up to order $k-1$, and the estimates on $\|\alpha\|_{\mathcal{C}_{0}^{k-1}}$ can be obtained by the standard energy method after an odd extension of the initial data to the whole $\mathbb{R}$.

To prove (2.6), note that

$$
\frac{\partial_{y} u^{s}(t, y+\bar{y})}{\partial_{y} u^{s}(t, y)}=\frac{\partial_{y} u^{s}(t, y+\bar{y})-\partial_{y} u^{s}(t, y)}{\partial_{y} u^{s}(t, y)}+1
$$

and

$$
\partial_{y} u^{s}(t, y+\bar{y})-\partial_{y} u^{s}(t, y)=\int_{0}^{\bar{y}} \partial_{y}^{2} u^{s}(t, y+z) d z .
$$

Hence, from (2.9), we have by using (2.5) that

$$
\left\|\frac{\partial_{y} u^{s}(t, y+\bar{y})}{\partial_{y} u^{s}(t, y)}\right\|_{\mathcal{C}_{0}^{k-1}\left([0, T] \times \mathbb{R}_{y}^{+}\right)} \leq 1+C \int_{0}^{\bar{y}}\left\|\frac{\partial_{y} u^{s}(t, y+z)}{\partial_{y} u^{s}(t, y)}\right\|_{\mathcal{C}_{0}^{k-1}\left([0, T] \times \mathbb{R}_{y}^{+}\right)} d z .
$$

By applying the Gronwall inequality to (2.10), it follows that

$$
\left\|\frac{\partial_{y} u^{s}(t, y+\bar{y})}{\partial_{y} u^{s}(t, y)}\right\|_{\mathcal{C}_{0}^{k-1}\left([0, T] \times \mathbb{R}_{y}^{+}\right)} \leq e^{C \bar{y}} .
$$

The estimate (2.7) can be proved similarly by noting that $\partial_{t} u^{s}=\partial_{y}^{2} u^{s}$.

Finally, we give the following remark on the condition (2.3) of the initial data $u_{0}^{s}(y)$ for the shear flow.

Remark 2.3. It is straightforward to show that the condition (2.3) is satisfied when $u_{0}^{s}(y)$ converges to 1 either exponentially or algebraically at the rates of $e^{-a y^{\alpha}}$ with $0<\alpha<\frac{1}{2}$, or $y^{-\beta}$ with $\beta>\max \left\{0, l-\frac{1}{2}\right\}$ at infinity. Here, $a>0$ is a constant, and $l>0$ is given in the proposition.

\section{Well-Posedness of the Linearized Prandtl Equation}

In this section, we study the well-posedness of a linearized problem of the Prandtl equation (1.2) in the Sobolev spaces by the energy method, when the background tangential velocity is monotonic in the normal variable. Again, the main novelty here is that unlike most of the previous works, the Crocco transformation will not be used.

In the estimates on the solutions to the linearized problem, we will see that there is a loss of regularity with respect to both the source term and the background state. And this inspires us to use the Nash-Moser-Hörmander iteration scheme to study the nonlinear Prandtl equation in the next section.

Let $(\tilde{u}, \tilde{v})$ be a smooth background state satisfying

$$
\partial_{y} \tilde{u}(t, x, y)>0, \quad \partial_{x} \tilde{u}+\partial_{y} \tilde{v}=0,
$$


and other conditions that will be specified later. Consider the following linearized problem of (1.2) around $(\tilde{u}, \tilde{v})$ :

$$
\left\{\begin{array}{l}
\partial_{t} u+\tilde{u} \partial_{x} u+\tilde{v} \partial_{y} u+u \partial_{x} \tilde{u}+v \partial_{y} \tilde{u}-\partial_{y}^{2} u=f, \\
\partial_{x} u+\partial_{y} v=0, \\
\left.u\right|_{y=0}=\left.v\right|_{y=0}=0, \quad \lim _{y \rightarrow+\infty} u(t, x, y)=0, \\
\left.u\right|_{t \leq 0}=0 .
\end{array}\right.
$$

Unlike using the Crocco transformation, our main idea is to rewrite the equations of (3.1) into a degenerate parabolic equation with an integral term without changing the independent variables, for which we can perform the energy estimates directly. For this purpose, we introduce the following change of the unknown function:

$$
w(t, x, y)=\left(\frac{u}{\partial_{y} \tilde{u}}\right)_{y}(t, x, y), \quad \text { that is, } \quad u(t, x, y)=\left(\partial_{y} \tilde{u}\right) \int_{0}^{y} w(t, x, \tilde{y}) d \tilde{y} .
$$

By a direct calculation, we get that for classical solutions, the problem (3.1) is equivalent to

$$
\left\{\begin{array}{l}
\partial_{t} w+\partial_{x}(\tilde{u} w)+\partial_{y}(\tilde{v} w)-2 \partial_{y}(\eta w)+\partial_{y}\left(\zeta \int_{0}^{y} w(t, x, \tilde{y}) d \tilde{y}\right)-\partial_{y}^{2} w=\partial_{y} \tilde{f} \\
\left.\left(\partial_{y} w+2 \eta w\right)\right|_{y=0}=-\left.\tilde{f}\right|_{y=0} \\
\left.w\right|_{t \leq 0}=0
\end{array}\right.
$$

where

$$
\eta=\frac{\partial_{y}^{2} \tilde{u}}{\partial_{y} \tilde{u}}, \quad \zeta=\frac{\left(\partial_{t}+\tilde{u} \partial_{x}+\tilde{v} \partial_{y}-\partial_{y}^{2}\right) \partial_{y} \tilde{u}}{\partial_{y} \tilde{u}}, \quad \tilde{f}=\frac{f}{\partial_{y} \tilde{u}} .
$$

To simplify the notations in the estimates on solutions to the problem (3.2), denote

$$
\begin{aligned}
\lambda_{k_{1}, k_{2}}= & \left\|\tilde{u}-u^{s}\right\|_{\mathcal{B}_{0,0}^{k_{1}, k_{2}}}+\left\|\partial_{\mathcal{T}}^{k_{1}} \partial_{y}^{k_{2}} u^{s}\right\|_{L_{t, y}^{2}}+\left\|\partial_{\mathcal{T}}^{k_{1}} \partial_{y}^{k_{2}} \tilde{v}\right\|_{L_{y}^{\infty}\left(L_{t, x}^{2}\right)} \\
& +\left\|\partial_{\mathcal{T}}^{k_{1}} \partial_{y}^{k_{2}} \bar{\eta}\right\|_{L_{y}^{2}\left(L_{t, x}^{\infty}\right)}+\|\eta-\bar{\eta}\|_{\mathcal{B}_{0,0}^{k_{1}, k_{2}}}+\|\zeta\|_{\mathcal{B}_{0, \ell}^{k_{1}, k_{2}}},
\end{aligned}
$$

and

$$
\lambda_{k}=\sum_{k_{1}+\left[\frac{k_{2}+1}{2}\right] \leq k} \lambda_{k_{1}, k_{2}}
$$

where

$$
\bar{\eta}=\frac{\partial_{y}^{2} u^{s}}{\partial_{y} \tilde{u}} .
$$

The following estimate for the problem (3.2) can be obtained by a direct energy method.

Theorem 3.1. For a given positive integer $k$, suppose that the compatibility conditions of the problem (3.2) hold up to order $k$. Then for any fixed $\ell>1 / 2$, we have the following estimate:

$$
\|w\|_{\mathcal{A}_{\ell}^{k}} \leq C_{1}\left(\lambda_{3}\right)\|\tilde{f}\|_{\mathcal{A}_{\ell}^{k}}+C_{2}\left(\lambda_{3}\right) \lambda_{k}\|\tilde{f}\|_{\mathcal{A}_{\ell}^{3}},
$$

where $C_{1}\left(\lambda_{3}\right), C_{2}\left(\lambda_{3}\right)$ are polynomials of $\lambda_{3}$ of order less than or equal to $k$. 
Remark 3.2. (1) It is easy to see that the compatibility conditions for the problem (3.2) up to order $k$ follow immediately from the corresponding conditions of the problem (3.1).

(2) From the estimate (3.4), one can easily deduce the estimates on the solution $(u, v)$ to the problem (3.1) in some weighted Sobolev spaces. Hence, from these estimates we can obtain the well-posedness of the linearized Prandtl equation in the Sobolev spaces.

The proof of Theorem 3.1 is based on the following lemmas.

Lemma 3.3 ( $L^{2}$-estimate). Under the assumptions of Theorem 3.1, for any fixed $T>0$, when $\lambda \geq\left(4 \ell\left(1+\lambda_{3}\right)\right)^{2}$ with $\lambda_{3}$ defined in (3.3), there is a constant $C(T)>0$ such that

$$
\|w\|_{\tilde{\mathcal{B}}_{\lambda, \ell}^{0,0}}^{2}+\lambda\|w\|_{\mathcal{B}_{\lambda, \ell}^{0,0}}^{2}+\left\|\partial_{y} w\right\|_{\mathcal{B}_{\lambda, \ell}^{0,0}}^{2} \leq C(T)\|\tilde{f}\|_{\mathcal{B}_{\lambda, \ell}^{0,0}}^{2}
$$

Proof. Multiplying (3.2) by $e^{-2 \lambda t}\langle y\rangle^{2 \ell} w$ and integrating over $\mathbb{R}_{+}^{2}$, we get

$$
\begin{aligned}
& \frac{1}{2} \partial_{t}\left\|e^{-\lambda t} w(t)\right\|_{L_{\ell}^{2}\left(\mathbb{R}_{+}^{2}\right)}^{2}+\lambda\left\|e^{-\lambda t} w(t)\right\|_{L_{\ell}^{2}\left(\mathbb{R}_{+}^{2}\right)}^{2}+\left\|e^{-\lambda t} \partial_{y} w(t)\right\|_{L_{\ell}^{2}\left(\mathbb{R}_{+}^{2}\right)}^{2} \\
& \leq\left(2 \ell+2\|\eta\|_{L^{\infty}}+\|\zeta\|_{L^{\infty}\left(\mathbb{R}_{x} ; L_{\ell}^{2}\left(\mathbb{R}_{+, y}\right)\right)}\right)\left\|e^{-\lambda t} \partial_{y} w(t)\right\|_{L_{\ell}^{2}\left(\mathbb{R}_{+}^{2}\right)}\left\|e^{-\lambda t} w(t)\right\|_{L_{\ell}^{2}\left(\mathbb{R}_{+}^{2}\right)} \\
& +\ell\|\tilde{v}\|_{L_{-1}^{\infty}}\left\|e^{-\lambda t} w(t)\right\|_{L_{\ell}^{2}\left(\mathbb{R}_{+}^{2}\right)}^{2}+2 \ell\left\|e^{-\lambda t} \tilde{f}(t)\right\|_{L_{\ell-1}^{2}\left(\mathbb{R}_{+}^{2}\right)}\left\|e^{-\lambda t} w(t)\right\|_{L_{\ell}^{2}\left(\mathbb{R}_{+}^{2}\right)} \\
& +\left\|e^{-\lambda t} \tilde{f}(t)\right\|_{L_{\ell}^{2}\left(\mathbb{R}_{+}^{2}\right)}\left\|e^{-\lambda t} \partial_{y} w(t)\right\|_{L_{\ell}^{2}\left(\mathbb{R}_{+}^{2}\right)},
\end{aligned}
$$

by using the boundary condition given in (3.2).

Using the classical Sobolev embedding theorem, it follows that

$$
4\left(2 \ell+2\|\eta\|_{L^{\infty}}+\|\zeta\|_{L_{t, x}^{\infty}\left(L_{\ell, y}^{2}\right)}\right)^{2}+2 \ell\|\tilde{v}\|_{L^{\infty}} \leq\left(4 \ell\left(1+\lambda_{3}\right)\right)^{2} .
$$

Thus, by taking $\lambda \geq\left(4 \ell\left(1+\lambda_{3}\right)\right)^{2}$, we get

$$
\begin{aligned}
& \partial_{t}\left\|e^{-\lambda t} w(t)\right\|_{L_{\ell}^{2}\left(\mathbb{R}_{+}^{2}\right)}^{2}+\lambda\left\|e^{-\lambda t} w(t)\right\|_{L_{\ell}^{2}\left(\mathbb{R}_{+}^{2}\right)}^{2}+\left\|e^{-\lambda t} \partial_{y} w(t)\right\|_{L_{\ell}^{2}\left(\mathbb{R}_{+}^{2}\right)}^{2} \\
& \quad \leq 4\left\|e^{-\lambda t} \tilde{f}(t)\right\|_{L_{\ell}^{2}\left(\mathbb{R}_{+}^{2}\right)}^{2},
\end{aligned}
$$

which implies

$$
\|w\|_{\tilde{\mathcal{B}}_{\lambda, \ell}^{0,0}}^{2}+\lambda\|w\|_{\mathcal{B}_{\lambda, \ell}^{0,0}}^{2}+\left\|\partial_{y} w\right\|_{\mathcal{B}_{\lambda, \ell}^{0,0}}^{2} \leq C(T)\|\tilde{f}\|_{\mathcal{B}_{\lambda, \ell}^{0,0}}^{2}
$$

for all fixed $0<T<+\infty$.

Lemma 3.4 (Energy estimate for tangential derivatives). Under the assumptions of Theorem 3.1, for any fixed $T>0$, there is a constant $C(T)>0$ such that

$$
\begin{aligned}
& \|w\|_{\tilde{\mathcal{B}}_{\lambda, \ell}^{k, 0}}^{2}+\lambda\|w\|_{\mathcal{B}_{\lambda, \ell}^{k, 0}}^{2}+\left\|\partial_{y} w\right\|_{\mathcal{B}_{\lambda, \ell}^{k, 0}}^{2} \\
& \leq C \\
& \quad C(T)\left(\|\tilde{f}\|_{\mathcal{B}_{\lambda, \ell}^{k, 0}}^{2}+\left(\|\zeta\|_{\mathcal{B}_{0, \ell}^{k, 0}}^{2}+\left\|\partial_{\mathcal{T}}^{k} \tilde{v}\right\|_{L_{y}^{\infty}\left(L_{t, x}^{2}\right)}^{2}+\left\|\partial_{\mathcal{T}}^{k} \bar{\eta}\right\|_{L_{y}^{2}\left(L_{t, x}^{\infty}\right)}^{2}\right)\|w\|_{\mathcal{B}_{\lambda, \ell}^{2,1}}^{2}\right. \\
& \left.\quad+\left(\left\|\tilde{u}-u^{s}\right\|_{\mathcal{B}_{0,0}^{k, 0}}^{2}+\left\|\partial_{\mathcal{T}}^{k} u^{s}\right\|_{L_{t, y}^{2}}^{2}+\|\eta-\bar{\eta}\|_{\mathcal{B}_{0,0}^{k, 0}}^{2}\right)\|w\|_{\mathcal{B}_{\lambda, \ell}^{3,1}}^{2}\right) .
\end{aligned}
$$

Proof. Taking the differentiation $\partial_{\mathcal{T}}^{\beta}(|\beta| \leq k)$ on the equation in (3.2), multiplying it by $e^{-2 \lambda t}\langle y\rangle^{2 \ell} \partial_{\mathcal{T}}^{\beta} w$, and integrating over $\mathbb{R}_{+}^{2}$, as in the proof of Lemma 3.3 , for 
$\lambda>\left(4 \ell\left(1+\lambda_{3}\right)\right)^{2}$, we have

$$
\begin{aligned}
& \partial_{t}\left\|e^{-\lambda t} \partial_{\mathcal{T}}^{\beta} w(t)\right\|_{L_{\ell}^{2}\left(\mathbb{R}_{+}^{2}\right)}^{2}+\lambda\left\|e^{-\lambda t} \partial_{\mathcal{T}}^{\beta} w(t)\right\|_{L_{\ell}^{2}\left(\mathbb{R}_{+}^{2}\right)}^{2}+\left\|e^{-\lambda t} \partial_{\mathcal{T}}^{\beta} \partial_{y} w(t)\right\|_{L_{\ell}^{2}\left(\mathbb{R}_{+}^{2}\right)}^{2} \\
& \quad \leq 4\left\|e^{-\lambda t} \partial_{\mathcal{T}}^{\beta} \tilde{f}(t)\right\|_{L_{\ell}^{2}}^{2}+A_{1}+A_{2}+A_{3},
\end{aligned}
$$

where we have used the compatibility conditions of the problem (3.2) and

$$
\left.\partial_{\mathcal{T}}^{\beta}(\tilde{v} w)\right|_{y=0}=0,
$$

by noting $\tilde{v}(t, x, y)=-\int_{0}^{y} \partial_{x} \tilde{u}(t, x, \tilde{y}) d \tilde{y}$, and $A_{1}, A_{2}$, and $A_{3}$ come from the commutators between $\partial_{\mathcal{T}}^{\beta}$ and the nonlinear terms in (3.2). For brevity, the precise definitions of $A_{i}, i=1,2,3$ are given respectively as follows.

First,

$$
\begin{aligned}
A_{1}= & \sum_{\beta_{1}+\beta_{2} \leq \beta ;\left|\beta_{2}\right|<|\beta|} C_{\beta}^{\beta_{1}}\left|\int_{\mathbb{R}_{+}^{2}} e^{-2 \lambda t}\langle y\rangle^{2 \ell}\left(\partial_{\mathcal{T}}^{\beta_{1}} \tilde{u}\right)\left(\partial_{\mathcal{T}}^{\beta_{2}} \partial_{x} w\right)\left(\partial_{\mathcal{T}}^{\beta} w\right) d x d y\right| \\
& +\sum_{\beta_{1}+\beta_{2} \leq \beta ;\left|\beta_{2}\right|<|\beta|} C_{\beta}^{\beta_{1}}\left|\int_{\mathbb{R}_{+}^{2}} e^{-2 \lambda t}\langle y\rangle^{2 \ell}\left(\partial_{\mathcal{T}}^{\beta_{1}} \tilde{v}\right)\left(\partial_{\mathcal{T}}^{\beta_{2}} w\right)\left(\partial_{\mathcal{T}}^{\beta} \partial_{y} w\right) d x d y\right|
\end{aligned}
$$

Therefore,

$$
\begin{aligned}
A_{1} \lesssim & \left\|e^{-\lambda t} \partial_{\mathcal{T}}^{\beta} w(t)\right\|_{L_{\ell}^{2}\left(\mathbb{R}_{+}^{2}\right)}\left\{\|\tilde{u}(t)\|_{L^{\infty}\left(\mathbb{R}_{+}^{2}\right)}\left\|e^{-\lambda t} \partial_{\mathcal{T}}^{|\beta|} w(t)\right\|_{L_{\ell}^{2}\left(\mathbb{R}_{+}^{2}\right)}\right. \\
& +\left\|\partial_{\mathcal{T}}^{|\beta|}\left(\tilde{u}(t)-u^{s}(t)\right)\right\|_{L^{2}\left(\mathbb{R}_{+}^{2}\right)}\left\|e^{-\lambda t} \partial_{x} w(t)\right\|_{L_{\ell}^{\infty}\left(\mathbb{R}_{+}^{2}\right)} \\
& \left.+\left\|\partial_{\mathcal{T}}^{|\beta|} u^{s}(t)\right\|_{L_{y}^{2}}\left\|e^{-\lambda t} \partial_{x} w(t)\right\|_{L_{y, \ell}^{\infty}\left(L_{x}^{2}\right)}\right\} \\
& +\left\|e^{-\lambda t} \partial_{\mathcal{T}}^{\beta} \partial_{y} w(t)\right\|_{L_{\ell}^{2}\left(\mathbb{R}_{+}^{2}\right)}\left\{\|\tilde{v}(t)\|_{L^{\infty}\left(\mathbb{R}_{+}^{2}\right)}\left\|e^{-\lambda t} \partial_{\mathcal{T}}^{|\beta|-1} w(t)\right\|_{L_{\ell}^{2}\left(\mathbb{R}_{+}^{2}\right)}\right. \\
& \left.+\left\|\partial_{\mathcal{T}}^{|\beta|} \tilde{v}(t)\right\|_{L_{y}^{\infty}\left(L_{x}^{2}\right)}\left\|e^{-\lambda t} w(t)\right\|_{L_{y, \ell}^{2}\left(L_{x}^{\infty}\right)}\right\}
\end{aligned}
$$

where we have used Lemma 2.1. Here and in the sequel, for simplicity, we shall use the notation $A \lesssim B$ when there exists a generic positive constant $C$ such that $A \leq C B$. A similar definition holds for $A \gtrsim B$.

Second, for

$$
A_{2}=\left|\int_{\mathbb{R}_{+}^{2}} e^{-2 \lambda}\langle y\rangle^{2 \ell} \partial_{\mathcal{T}}^{\beta}(\eta w)\left(\partial_{\mathcal{T}}^{\beta} \partial_{y} w\right) d x d y\right|
$$

we have

$$
\begin{aligned}
A_{2} \lesssim & \|\eta(t)\|_{L^{\infty}\left(\mathbb{R}_{+}^{2}\right)}\left\|e^{-\lambda t} \partial_{\mathcal{T}}^{|\beta|} w(t)\right\|_{L_{\ell}^{2}\left(\mathbb{R}_{+}^{2}\right)}\left\|e^{-\lambda t} \partial_{\mathcal{T}}^{\beta} \partial_{y} w(t)\right\|_{L_{\ell}^{2}\left(\mathbb{R}_{+}^{2}\right)} \\
& +\left\|\partial_{\mathcal{T}}^{|\beta|}(\eta-\bar{\eta})(t)\right\|_{L^{2}\left(\mathbb{R}_{+}^{2}\right)}\left\|e^{-\lambda t} w(t)\right\|_{L_{\ell}^{\infty}\left(\mathbb{R}_{+}^{2}\right)}\left\|e^{-\lambda t} \partial_{\mathcal{T}}^{\beta} \partial_{y} w(t)\right\|_{L_{\ell}^{2}\left(\mathbb{R}_{+}^{2}\right)} \\
& +\left\|\partial_{\mathcal{T}}^{|\beta|} \bar{\eta}(t)\right\|_{L^{\infty}\left(\mathbb{R}_{+}^{2}\right)}\left\|e^{-\lambda t} w(t)\right\|_{L_{\ell}^{2}\left(\mathbb{R}_{+}^{2}\right)}\left\|e^{-\lambda t} \partial_{\mathcal{T}}^{\beta} \partial_{y} w(t)\right\|_{L_{\ell}^{2}\left(\mathbb{R}_{+}^{2}\right)}
\end{aligned}
$$

Finally, for

$$
A_{3}=\left|\int_{\mathbb{R}_{+}^{2}} e^{-2 \lambda}\langle y\rangle^{2 \ell} \partial_{\mathcal{T}}^{\beta}\left(\zeta \int_{0}^{y} w d \tilde{y}\right)\left(\partial_{\mathcal{T}}^{\beta} \partial_{y} w\right) d x d y\right|,
$$


we get for $\ell>1 / 2$,

$$
\begin{aligned}
A_{3} \lesssim & \|\zeta(t)\|_{L_{y, \ell}^{2}\left(L_{x}^{\infty}\right)}\left\|e^{-\lambda t} \partial_{\mathcal{T}}^{|\beta|} w(t)\right\|_{L_{\ell}^{2}\left(\mathbb{R}_{+}^{2}\right)}\left\|e^{-\lambda t} \partial_{\mathcal{T}}^{\beta} \partial_{y} w(t)\right\|_{L_{\ell}^{2}\left(\mathbb{R}_{+}^{2}\right)} \\
& +\left\|\partial_{\mathcal{T}}^{|\beta|} \zeta(t)\right\|_{L_{\ell}^{2}\left(\mathbb{R}_{+}^{2}\right)}\left\|e^{-\lambda t} w(t)\right\|_{L_{y, \ell}^{2}\left(L_{x}^{\infty}\right)}\left\|e^{-\lambda t} \partial_{\mathcal{T}}^{\beta} \partial_{y} w(t)\right\|_{L_{\ell}^{2}\left(\mathbb{R}_{+}^{2}\right)}
\end{aligned}
$$

Substituting these estimates of $A_{1}, A_{2}, A_{3}$ into (3.6), and taking the summation over all $|\beta| \leq k$, it follows that

$$
\begin{aligned}
& \partial_{t}\left\|e^{-\lambda t} \partial_{\mathcal{T}}^{k} w(t)\right\|_{L_{\ell}^{2}\left(\mathbb{R}_{+}^{2}\right)}^{2}+\lambda\left\|e^{-\lambda t} \partial_{\mathcal{T}}^{k} w(t)\right\|_{L_{\ell}^{2}\left(\mathbb{R}_{+}^{2}\right)}^{2}+\left\|e^{-\lambda t} \partial_{\mathcal{T}}^{k} \partial_{y} w(t)\right\|_{L_{\ell}^{2}\left(\mathbb{R}_{+}^{2}\right)}^{2} \\
& \lesssim\left\|e^{-\lambda t} \partial_{\mathcal{T}}^{k} \tilde{f}(t)\right\|_{L_{\ell}^{2}}^{2}+\left\|\partial_{\mathcal{T}}^{k}\left(\tilde{u}-u^{s}\right)\right\|_{L^{2}\left(\mathbb{R}_{+}^{2}\right)}^{2}\left\|e^{-\lambda t} \partial_{x} w(t)\right\|_{L_{\ell}^{\infty}\left(\mathbb{R}_{+}^{2}\right)}^{2} \\
&+\left\|\partial_{\mathcal{T}}^{k} u^{s}\right\|_{L_{y}^{2}}^{2}\left\|e^{-\lambda t} \partial_{x} w(t)\right\|_{L_{y, \ell}^{\infty}\left(L_{x}^{2}\right)}^{2}+\left\|\partial_{\mathcal{T}}^{k} \tilde{v}\right\|_{L_{y}^{\infty}\left(L_{x}^{2}\right)}^{2}\left\|e^{-\lambda t} w(t)\right\|_{L_{y, \ell}^{2}\left(L_{x}^{\infty}\right)}^{2} \\
&+\left\|\partial_{\mathcal{T}}^{k} \bar{\eta}(t)\right\|_{L_{y}^{2}\left(L_{x}^{\infty}\right)}^{2}\left\|e^{-\lambda t} w(t)\right\|_{L_{y, \ell}^{\infty}\left(L_{x}^{2}\right)}^{2}+\left\|\partial_{\mathcal{T}}^{k}(\eta-\bar{\eta})(t)\right\|_{L^{2}\left(\mathbb{R}_{+}^{2}\right)}^{2}\left\|e^{-\lambda t} w(t)\right\|_{L_{\ell}^{\infty}\left(\mathbb{R}_{+}^{2}\right)}^{2} \\
&+\left\|\partial_{\mathcal{T}}^{k} \zeta(t)\right\|_{L_{\ell}^{2}\left(\mathbb{R}_{+}^{2}\right)}^{2}\left\|e^{-\lambda t} w(t)\right\|_{L_{y, \ell}^{2}\left(L_{x}^{\infty}\right)}^{2},
\end{aligned}
$$

for any $\lambda>\left(4 \ell\left(1+\lambda_{3}\right)\right)^{2}$. Integrating the above inequality on $[0, T]$, and using the compatibility condition

$$
\left.\left(\partial_{t, x, y}^{k} w\right)\right|_{t=0}=0
$$

we get for any fixed $T>0$,

$$
\begin{aligned}
& \|w\|_{\tilde{\mathcal{B}}_{\lambda, \ell}^{k, 0}}^{2}+\lambda\|w\|_{\mathcal{B}_{\lambda, \ell}^{k, 0}}^{2}+\left\|\partial_{y} w\right\|_{\mathcal{B}_{\lambda, \ell}^{k, 0}}^{2} \leq C(T)\left\{\|\tilde{f}\|_{\mathcal{B}_{\lambda, \ell}^{k, 0}}^{2}\right. \\
& \quad+\left(\|\zeta\|_{\mathcal{B}_{0, \ell}^{k, 0}}^{2}+\left\|\partial_{\mathcal{T}}^{k} \tilde{v}\right\|_{L^{2}\left([0, T] \times \mathbb{R}_{x} ; L^{\infty}\left(\mathbb{R}_{+}\right)\right)}^{2}\right)\left\|e^{-\lambda t} w\right\|_{L^{\infty}\left([0, T] \times \mathbb{R}_{x} ; L_{y, \ell}^{2}\left(\mathbb{R}_{+}\right)\right)}^{2} \\
& \quad+\left\|\tilde{u}-u^{s}\right\|_{\mathcal{B}_{0,0}^{k, 0}}^{2}\left\|e^{-\lambda t} \partial_{x} w\right\|_{L_{\ell}^{\infty}\left(\mathbb{R}_{+}^{2}\right)}^{2}+\left\|\partial_{\mathcal{T}}^{k} u^{s}\right\|_{L_{t, y}^{2}}^{2}\left\|e^{-\lambda t} \partial_{x} w\right\|_{L_{y, \ell}^{\infty}\left(L^{\infty}\left([0, T], L_{x}^{2}(\mathbb{R})\right)\right)}^{2}\left\|\left.\right|^{-\lambda t} w\right\|_{L_{y, \ell}^{\infty}\left(L_{t, x}^{2}\right)}^{2} \\
& \quad+\left\|\partial_{\mathcal{T}}^{k} \bar{\eta}\right\|_{L_{y}^{2}\left(L_{t, x}^{\infty}\right)}^{2} \| e^{-\lambda} \\
& \left.\quad+\|\eta-\bar{\eta}\|_{\mathcal{B}_{0,0}^{k, 0}}^{2}\left\|e^{-\lambda t} w\right\|_{L_{\ell}^{\infty}\left([0, T] \times \mathbb{R}_{+}^{2}\right)}^{2}\right\}
\end{aligned}
$$

Using the Sobolev embedding theorem in the above inequality, the estimate (3.5) follows immediately.

Remark 3.5. The estimate (3.5) implies

$$
\|w\|_{\mathcal{B}_{\lambda, \ell}^{k, 1}}^{2} \lesssim\|\tilde{f}\|_{\mathcal{B}_{\lambda, \ell}^{k, 0}}^{2}+\lambda_{k, 0}^{2}\|w\|_{\mathcal{B}_{\lambda, \ell}^{3,1}}^{2}
$$

Moreover, using the same argument as in the above proof together with Lemma 3.3 , when $\lambda>\left(4 \ell\left(1+\lambda_{3}\right)\right)^{2}$, we can obtain

$$
\|w\|_{\mathcal{B}_{\lambda, \ell}^{k, 1}} \lesssim\|\tilde{f}\|_{\mathcal{B}_{\lambda, \ell}^{k, \ell}}, \quad 0 \leq k \leq 3 .
$$

Proof of Theorem 3.1, Recall from (3.2) that

$$
\partial_{y}^{2} w=\partial_{t} w+\tilde{u} \partial_{x} w+\tilde{v} \partial_{y} w-2 \partial_{y}(\eta w)+\partial_{y}\left(\zeta \int_{0}^{y} w(t, x, \tilde{y}) d \tilde{y}\right)-\partial_{y} \tilde{f}
$$


By applying $\partial_{\mathcal{T}}^{k_{1}}$ to this equation and using Lemma 2.1, we get

$$
\begin{aligned}
\|w\|_{\mathcal{B}_{\lambda, \ell}^{k_{1}, 2}} \leq & \|w\|_{\mathcal{B}_{\lambda, \ell}^{k_{1}+1,0}}+\left\|\tilde{u} \partial_{x} w\right\|_{\mathcal{B}_{\lambda, \ell}^{k_{1}, 0}}+\left\|\tilde{v} \partial_{y} w\right\|_{\mathcal{B}_{\lambda, \ell}^{k_{1}, 0}}+2\|\eta w\|_{\mathcal{B}_{\lambda, \ell}^{k_{1}, 1}} \\
& +\left\|\left(\partial_{y} \zeta\right) \int_{0}^{y} w(t, x, \tilde{y}) d \tilde{y}\right\|_{\mathcal{B}_{\lambda, \ell}^{k_{1}, 0}}+\|\zeta w\|_{\mathcal{B}_{\lambda, \ell}^{k_{1}, 0}}+\left\|\partial_{y} \tilde{f}\right\|_{\mathcal{B}_{\lambda, \ell}^{k_{1}, 0}} \\
\lesssim & \left(1+\|\tilde{u}\|_{L^{\infty}}\right)\|w\|_{\mathcal{B}_{\lambda, \ell}^{k_{1}+1,0}}+\left(\|\tilde{v}\|_{L^{\infty}}+\|\eta\|_{\left.L^{\infty}\right)}\right)\|w\|_{\mathcal{B}_{\lambda, \ell}^{k_{1}, 1}} \\
& +\|\zeta\|_{L^{\infty}}\|w\|_{\mathcal{B}_{\lambda, \ell}^{k_{1}, 0}}+\left\|\tilde{u}-u^{s}\right\|_{\mathcal{B}_{0,0}^{k_{1}, 0}}\left\|\partial_{x} w\right\|_{L_{\lambda, \ell}^{\infty}} \\
& +\left\|\partial_{\mathcal{T}}^{k_{1}} u^{s}\right\|_{L_{y}^{2}\left(L_{t}^{\infty}\right)}\left\|\partial_{x} w\right\|_{L_{y, \ell}^{\infty}\left(L_{t, x, \lambda}^{2}\right)}+\left\|\partial_{\mathcal{T}}^{k_{1}} \tilde{v}\right\|_{L_{y}^{\infty}\left(L_{t, x}^{2}\right)}\left\|\partial_{y} w\right\|_{L_{y, \ell}^{2}\left(L_{t, x, \lambda}^{\infty}\right)} \\
& +\left\|\partial_{\mathcal{T}}^{k_{1}} \partial_{y} \bar{\eta}\right\|_{L_{y}^{2}\left(L_{t, x}^{\infty}\right)}\|w\|_{L_{y, \ell}^{\infty}\left(L_{t, x, \lambda}^{2}\right)}+\|\eta-\bar{\eta}\|_{\mathcal{B}_{0,0}^{k_{1}, 1}}\|w\|_{L_{\lambda, \ell}^{\infty}} \\
& +\left\|\partial_{y} \tilde{f}\right\|_{\mathcal{B}_{\lambda, \ell}^{k_{1}, 0}}+\left\|\partial_{y} \zeta\right\|_{L_{t, x}^{\infty}\left(L_{\ell}^{2}\right)}\|w\|_{\mathcal{B}_{\lambda, \ell}^{k_{1}, 0}}+\|\zeta\|_{\mathcal{B}_{0, \ell}^{k_{1}, 1}}\|w\|_{L_{t, x}^{\infty}\left(L_{\lambda, \ell}^{2}\right)} .
\end{aligned}
$$

Then by using the Sobolev embedding theorem, it follows that

$$
\|w\|_{\mathcal{B}_{\lambda, \ell}^{k_{1}, 2}} \lesssim \lambda_{2,1}\left(\|w\|_{\mathcal{B}_{\lambda, \ell}^{k_{1}+1,0}}+\|w\|_{\mathcal{B}_{\lambda, \ell}^{k_{1}, 1}}\right)+\left\|\partial_{y} \tilde{f}\right\|_{\mathcal{B}_{\lambda, \ell}^{k_{1}, 0}}+\lambda_{k_{1}, 1}\|w\|_{\mathcal{B}_{\lambda, \ell}^{3,1}} .
$$

And from (3.7) and (3.8), we get

$$
\|w\|_{\mathcal{B}_{\lambda, \ell}^{k_{1}, 2}} \lesssim \lambda_{2,1}\left(\|\tilde{f}\|_{\mathcal{B}_{\lambda, \ell}^{k_{1}+1,0}}+\|\tilde{f}\|_{\mathcal{B}_{\lambda, \ell}^{k_{1}, 1}}\right)+\left(\lambda_{2,1} \lambda_{k_{1}+1,0}+\lambda_{k_{1}, 1}\right)\|\tilde{f}\|_{\mathcal{B}_{\lambda, \ell}^{3,0}}
$$

For $k_{2}>2$, differentiating Equation (3.9) by $\partial_{\mathcal{T}}^{k_{1}} \partial_{y}^{k_{2}-2}$, we have

$$
\begin{aligned}
& \|w\|_{\mathcal{B}_{\lambda, \ell}^{k_{1}, k_{2}}} \leq\|w\|_{\mathcal{B}_{\lambda, \ell}^{k_{1}+1, k_{2}-2}}+\left\|\tilde{u} \partial_{x} w\right\|_{\mathcal{B}_{\lambda, \ell}^{k_{1}, k_{2}-2}}+\left\|\tilde{v} \partial_{y} w\right\|_{\mathcal{B}_{\lambda, \ell}^{k_{1}, k_{2}-2}} \\
& +2\|\eta w\|_{\mathcal{B}_{\lambda, \ell}^{k_{1}, k_{2}-1}}+\left\|\left(\partial_{y} \zeta\right) \int_{0}^{y} w(t, x, \tilde{y}) d \tilde{y}\right\|_{\mathcal{B}_{\lambda, \ell}^{k_{1}, k_{2}-2}} \\
& +\|\zeta w\|_{\mathcal{B}_{\lambda, \ell}^{k_{1}, k_{2}-2}}+\left\|\partial_{y} \tilde{f}\right\|_{\mathcal{B}_{\lambda, \ell}^{k_{1}, k_{2}-2}} \\
& \lesssim\left(1+\|\tilde{u}\|_{L^{\infty}}\right)\|w\|_{\mathcal{B}_{\lambda, \ell}^{k_{1}+1, k_{2}-2}}+\left(\|\tilde{v}\|_{L^{\infty}}+\|\eta\|_{L^{\infty}}\right)\|w\|_{\mathcal{B}_{\lambda, \ell}^{k_{1}, k_{2}-1}} \\
& +\|\zeta\|_{L^{\infty}}\|w\|_{\mathcal{B}_{\lambda, \ell}^{k_{1}, k_{2}-2}}+\left\|\partial_{y} \zeta\right\|_{L_{t, x}^{\infty}\left(L_{\ell}^{2}\right)}\|w\|_{\mathcal{B}_{\lambda, \ell}^{k_{1}, k_{2}-2}}+\left\|\partial_{y} \tilde{f}\right\|_{\mathcal{B}_{\lambda, \ell}^{k_{1}, k_{2}-2}} \\
& +\left\|\tilde{u}-u^{s}\right\|_{\mathcal{B}_{0,0}^{k_{1}, k_{2}-2}}\left\|\partial_{x} w\right\|_{L_{\lambda, \ell}^{\infty}}+\left\|\partial_{\mathcal{T}}^{k_{1}} \partial_{y}^{k_{2}-2} u^{s}\right\|_{L_{y}^{2}\left(L_{t}^{\infty}\right)}\left\|\partial_{x} w\right\|_{L_{y, \ell}^{\infty}\left(L_{t, x, \lambda}^{2}\right)} \\
& +\left\|\partial_{\mathcal{T}}^{k_{1}} \partial_{y}^{k_{2}-2} \tilde{v}\right\|_{L_{y}^{\infty}\left(L_{t, x}^{2}\right)}\left\|\partial_{y} w\right\|_{L_{y, \ell}^{2}\left(L_{t, x, \lambda}^{\infty}\right)} \\
& +\left\|\partial_{\mathcal{T}}^{k_{1}} \partial_{y}^{k_{2}-1} \bar{\eta}\right\|_{L_{y}^{2}\left(L_{t, x}^{\infty}\right)}\|w\|_{L_{y, \ell}^{\infty}\left(L_{t, x, \lambda}^{2}\right)}+\|\eta-\bar{\eta}\|_{\mathcal{B}_{0,0}^{k_{1}, k_{2}-1}}\|w\|_{L_{\lambda, \ell}^{\infty}} \\
& +\|\zeta\|_{\mathcal{B}_{0, \ell}^{k_{1}, k_{2}-1}}\|w\|_{L_{y, \ell}^{\infty}\left(L_{t, x, \lambda}^{2}\right)} .
\end{aligned}
$$

Therefore, we get

$$
\|w\|_{\mathcal{B}_{\lambda, \ell}^{k_{1}, k_{2}}} \lesssim \lambda_{2,1}\left(\|w\|_{\mathcal{B}_{\lambda, \ell}^{k_{1}+1, k_{2}-2}}+\|w\|_{\mathcal{B}_{\lambda, \ell}^{k_{1}, k_{2}-1}}\right)+\lambda_{k_{1}, k_{2}-1}\|w\|_{\mathcal{B}_{\lambda, \ell}^{3,1}}+\|\tilde{f}\|_{\mathcal{B}_{\lambda, \ell}^{k_{1}, k_{2}-1}}
$$

which immediately implies

$$
\|w\|_{\mathcal{B}_{0, \ell}^{k_{1}, k_{2}}} \lesssim \lambda_{2,1}\left(\|w\|_{\mathcal{B}_{0, \ell}^{k_{1}+1, k_{2}-2}}+\|w\|_{\mathcal{B}_{0, \ell}^{k_{1}, k_{2}-1}}\right)+\lambda_{k_{1}, k_{2}-1}\|w\|_{\mathcal{B}_{0, \ell}^{3,1}}+\|\tilde{f}\|_{\mathcal{B}_{0, \ell}^{k_{1}, k_{2}-1}},
$$

by fixing $\lambda>\left(4 \ell\left(1+\lambda_{3}\right)\right)^{2}$.

The proof of Theorem 3.1 can then be completed by induction on $k_{2}$. 


\section{Iteration SCheme For the nOnlinear Prandtl EQUation}

From the estimate (3.4) given in Theorem 3.1, we see that there is a loss of regularity in the solutions to the linearized Prandtl equation with respect to the source term and the background state. In order to take care of this loss, we are going to apply the Nash-Moser-Hörmander iteration scheme (cf. [1, 10, 13, 16, 17]) to study the nonlinear problem (1.2).

4.1. The smoothing operators. For a function $f$ defined on $\Omega=\left[0,+\infty\left[\times \mathbb{R}_{x} \times\right.\right.$ $\mathbb{R}_{y}^{+}$, let $\tilde{f}$ be its extension to $\mathbb{R}^{3}$ by 0 . Then for a large constant $\theta$, introduce a family of smoothing operators $S_{\theta}$,

$$
\left(S_{\theta} f\right)(t, x, y)=\int \rho_{\theta}(\tau) \rho_{\theta}(\xi) \rho_{\theta}(\eta) \tilde{f}\left(t-\tau+\theta^{-1}, x-\xi, y-\eta+\theta^{-1}\right) d \tau d \xi d \eta,
$$

where $\rho_{\theta}(\tau)=\theta \rho(\theta \tau), \rho \in C_{0}^{\infty}(\mathbb{R})$ with Supp $\rho \subseteq[-1,1]$ and $\|\rho\|_{L^{1}}=1$. One has

$$
\left\{S_{\theta}\right\}_{\theta>0}: \mathcal{A}_{\ell}^{0}(\Omega) \longrightarrow \cap_{s \geq 0} \mathcal{A}_{\ell}^{s}(\Omega)
$$

together with

$$
\left\{\begin{array}{l}
\left\|S_{\theta} u\right\|_{\mathcal{A}_{\ell}^{s}} \leq C_{\rho} \theta^{(s-\alpha)+}\|u\|_{\mathcal{A}_{\ell}^{\alpha}}, \quad \text { for all } s, \alpha \geq 0, \\
\left\|\left(1-S_{\theta}\right) u\right\|_{\mathcal{A}_{\ell}^{s}} \leq C_{\rho} \theta^{s-\alpha}\|u\|_{\mathcal{A}_{\ell}^{\alpha}}, \quad \text { for all } 0 \leq s \leq \alpha,
\end{array}\right.
$$

where the constant $C_{\rho}$ depends only on the function $\rho$ and the orders of differentiations $s$ and $\alpha$. For the smoothing parameter, we set $\theta_{n}=\sqrt{\theta_{0}^{2}+n}$ for any $n \geq 1$ and a large fixed constant $\theta_{0}$. We have also

$$
\left\|\left(S_{\theta_{n}}-S_{\theta_{n-1}}\right) u\right\|_{\mathcal{A}_{\ell}^{s}} \leq C_{\rho} \theta_{n}^{s-\alpha} \Delta \theta_{n}\|u\|_{\mathcal{A}_{\ell}^{\alpha}}, \quad \text { for all } s, \alpha \geq 0,
$$

where $\Delta \theta_{n}=\theta_{n+1}-\theta_{n}$.

The operator $S_{\theta}$ acting on the other three spaces introduced in Section 2.1 shares the same properties.

The following commutator estimates will be used frequently later,

Lemma 4.1. For any proper function $f$, we have

$$
\left\|\left[\frac{1}{\partial_{y} u^{s}}, S_{\theta}\right]\left(\partial_{y} f\right)\right\|_{\mathcal{A}_{\ell}^{k}} \leq C_{k}\left\|\frac{f}{\partial_{y} u^{s}}\right\|_{\mathcal{A}_{\ell}^{k}},
$$

and

$$
\left\|\partial_{y}\left[\frac{1}{\partial_{y} u^{s}}, \partial_{y} S_{\theta}\right] f\right\|_{\mathcal{A}_{\ell}^{k}} \leq C_{k} \theta\left\|\frac{f}{\partial_{y} u^{s}}\right\|_{\mathcal{A}_{\ell}^{k}},
$$

where the constant $C_{k}$ depends on the constant in (2.5). Similar inequalities hold for the norms $\|\cdot\|_{\mathcal{B}_{\ell}^{k_{1}, k_{2}}},\|\cdot\|_{\mathcal{C}_{\ell}^{k}}$ and $\|\cdot\|_{\mathcal{D}_{\ell}^{k}}$.

Proof. This lemma can be proved in a classical way; cf. 3] 12. To be self-contained, we give a brief proof of the estimate (4.3) here. Note that (4.4) can be proved similarly. 
From the definition, we have

$$
\begin{aligned}
{\left[\frac{1}{\partial_{y} u^{s}}, S_{\theta}\right] b=} & \frac{S_{\theta}(b)}{\partial_{y} u^{s}}(t, x, y)-S_{\theta}\left(\frac{b}{\partial_{y} u^{s}}\right)(t, x, y) \\
= & \int \rho_{\theta}(\tau) \rho_{\theta}(\xi) \rho_{\theta}(\eta)\left(\frac{\partial_{y} u^{s}\left(t-\tau+\theta^{-1}, y-\eta+\theta^{-1}\right)-\partial_{y} u^{s}(t, y)}{\partial_{y} u^{s}(t, y)}\right) \\
& \times\left(\frac{\tilde{b}}{\partial_{y} u^{s}}\right)\left(t-\tau+\theta^{-1}, x-\xi, y-\eta+\theta^{-1}\right) d \tau d \xi d \eta \\
= & \theta^{-1} \int \rho_{\theta}(\xi)\left(\tilde{\rho}_{\theta}(\tau) \rho_{\theta}(\eta) a_{1}(t, \tau, y, \eta, \theta)+\rho_{\theta}(\tau) \tilde{\rho}_{\theta}(\eta) a_{2}(t, \tau, y, \eta, \theta)\right) \\
& \times\left(\frac{\tilde{b}}{\partial_{y} u^{s}}\right)\left(t-\tau+\theta^{-1}, x-\xi, y-\eta+\theta^{-1}\right) d \tau d \xi d \eta,
\end{aligned}
$$

with $\tilde{\rho}_{\theta}(\tau)=\theta \rho(\theta \tau)(1-\theta \tau), \tilde{\rho}_{\theta}(\eta)=\theta \rho(\theta \eta)(1-\theta \eta)$, and

$$
a_{1}(t, \tau, y, \eta, \theta)=-\int_{0}^{1} \frac{\partial_{t} \partial_{y} u^{s}\left(t+\lambda\left(\theta^{-1}-\tau\right), y-\eta+\theta^{-1}\right)}{\partial_{y} u^{s}(t, y)} d \lambda,
$$

and

$$
a_{2}(t, \tau, y, \eta, \theta)=-\int_{0}^{1} \frac{\partial_{y}^{2} u^{s}\left(t, y+\lambda\left(\theta^{-1}-\eta\right)\right)}{\partial_{y} u^{s}(t, y)} d \lambda .
$$

Using (2.6) and (2.7), for $j=1,2$, we have

$$
\sup _{0 \leq|\tau|,|\eta| \leq \theta^{-1} \leq R_{0}}\left\|a_{j}(\cdot, \tau, \cdot, \eta, \theta)\right\|_{\mathcal{C}_{0}^{k+1}} \leq \tilde{C}(T) .
$$

Thus, from (4.5) we get the estimate (4.3) immediately.

4.2. The iteration scheme. Denote by

$$
\mathcal{P}(u, v)=\partial_{t} u+u \partial_{x} u+v \partial_{y} u-\partial_{y}^{2} u,
$$

the nonlinear operator associated with problem (1.2), and its linearized operator around $(\tilde{u}, \tilde{v})$ by

$$
\mathcal{P}_{(\tilde{u}, \tilde{v})}^{\prime}(u, v)=\partial_{t} u+\tilde{u} \partial_{x} u+\tilde{v} \partial_{y} u+u \partial_{x} \tilde{u}+v \partial_{y} \tilde{u}-\partial_{y}^{2} u .
$$

In this subsection, we introduce an iteration scheme in order to construct an approximate solution sequence $\left\{\left(u^{n}, v^{n}\right)\right\}$ to the problem (1.2).

For a fixed integer $k \geq 0$, suppose that the initial data in the Prandtl equation (1.2) satisfies the compatibility conditions up to order $k$, and that $(u, v)$ is a classical solution. If we set $\tilde{u}=u-u^{s}$ with $u^{s}(t, y)$ being the heat profile defined in Section 2.2 , then it is easy to see that

$$
\left\{\begin{array}{l}
\partial_{t} \tilde{u}+\left(\tilde{u}+u^{s}\right) \tilde{u}_{x}+v \partial_{y}\left(\tilde{u}+u^{s}\right)-\tilde{u}_{y y}=0, \quad(x, y) \in \mathbb{R}_{+}^{2}, t>0 \\
\partial_{x} \tilde{u}+\partial_{y} v=0 \\
\left.\tilde{u}\right|_{y=0}=\left.v\right|_{y=0}=0, \quad \lim _{y \rightarrow+\infty} \tilde{u}=0 \\
\left.\tilde{u}\right|_{t=0}=\tilde{u}_{0}(x, y) .
\end{array}\right.
$$

The compatibility conditions for (4.7) follow from those for (1.2) immediately. 
The zeroth order approximate solution: Denote

$$
\tilde{u}_{0}^{j}(x, y)=\left.\partial_{t}^{j} \tilde{u}\right|_{t=0}, \quad v_{0}^{j}(x, y)=\left.\partial_{t}^{j} v\right|_{t=0} .
$$

Then from the compatibility conditions for (4.7), $\left\{\tilde{u}_{0}^{j}, v_{0}^{j}\right\}_{j \leq k}$ are defined directly by $\tilde{u}_{0}(x, y)$. We are going to construct the zero-th order approximate solution $\left(\tilde{u}^{0}, v^{0}\right)$ of (4.7), such that

$$
\left.\partial_{t}^{j} \tilde{u}^{0}\right|_{t=0}=\tilde{u}_{0}^{j}(x, y),\left.\quad \partial_{t}^{j} v^{0}\right|_{t=0}=v_{0}^{j}(x, y), \quad 0 \leq j \leq k,
$$

and $\left(u^{0}, v^{0}\right)=\left(u^{s}+\tilde{u}^{0}, v^{0}\right)$ satisfying

$$
\left\{\begin{array}{l}
\partial_{x} u^{0}+\partial_{y} v^{0}=0, \quad(x, y) \in \mathbb{R}_{+}^{2}, t \geq 0 \\
\left.u^{0}\right|_{y=0}=\left.v^{0}\right|_{y=0}=0, \lim _{y \rightarrow+\infty} u^{0}=1 \\
\left.u^{0}\right|_{t=0}=u_{0}(x, y)
\end{array}\right.
$$

Other properties of $\left(u^{0}, v^{0}\right)$ will be studied in more detail in Section [5.1.

The Nash-Moser iteration scheme: Assume that for all $k=0, \ldots, n$, we have constructed the approximate solutions $\left(u^{k}, v^{k}\right)$ of (1.2) satisfying the same conditions given in (4.8) for $\left(u^{0}, v^{0}\right)$. We now construct the $(n+1)$-th approximation solution $\left(u^{n+1}, v^{n+1}\right)$ as follows. Set

$$
u^{n+1}=u^{n}+\delta u^{n}=u^{s}+\tilde{u}^{n}+\delta u^{n}, \quad v^{n+1}=v^{n}+\delta v^{n},
$$

where the increment $\left(\delta u^{n}, \delta v^{n}\right)$ is the solution of the following initial-boundary value problem:

$$
\left\{\begin{array}{l}
\mathcal{P}_{\left(u_{\theta_{n}}^{n}, v_{\theta_{n}}^{n}\right)}^{\prime}\left(\delta u^{n}, \delta v^{n}\right)=f^{n} \\
\partial_{x}\left(\delta u^{n}\right)+\partial_{y}\left(\delta v^{n}\right)=0, \\
\left.\delta u^{n}\right|_{y=0}=\left.\delta v^{n}\right|_{y=0}=0, \quad \lim _{y \rightarrow+\infty} \delta u^{n}=0 \\
\left.\delta u^{n}\right|_{t \leq 0}=0
\end{array}\right.
$$

where $u_{\theta_{n}}^{n}=u^{s}+S_{\theta_{n}} \tilde{u}^{n}$ and $v_{\theta_{n}}^{n}=S_{\theta_{n}} v^{n}$.

Now, we define the source term $f^{n}$ for the problem (4.10) in order to have the convergence of the approximate solution sequence $\left(u^{n}, v^{n}\right)$ to the solution of the Prandtl equation (1.2) as $n$ goes to infinity. Obviously, we have the following identity:

$$
\mathcal{P}\left(u^{n+1}, v^{n+1}\right)-\mathcal{P}\left(u^{n}, v^{n}\right)=\mathcal{P}_{\left(u_{\theta_{n}}^{n}, v_{\theta_{n}}^{n}\right)}^{\prime}\left(\delta u^{n}, \delta v^{n}\right)+e_{n},
$$

where

$$
e_{n}=e_{n}^{(1)}+e_{n}^{(2)}
$$

Here

$$
\begin{aligned}
e_{n}^{(1)} & =\mathcal{P}\left(u^{n}+\delta u^{n}, v^{n}+\delta v^{n}\right)-\mathcal{P}\left(u^{n}, v^{n}\right)-\mathcal{P}_{\left(u^{n}, v^{n}\right)}^{\prime}\left(\delta u^{n}, \delta v^{n}\right) \\
& =\delta u^{n} \partial_{x}\left(\delta u^{n}\right)+\delta v^{n} \partial_{y}\left(\delta u^{n}\right)
\end{aligned}
$$


is the error from the Newton iteration scheme, and

$$
\begin{aligned}
e_{n}^{(2)}= & \mathcal{P}_{\left(u^{n}, v^{n}\right)}^{\prime}\left(\delta u^{n}, \delta v^{n}\right)-\mathcal{P}_{\left(u_{\theta_{n}}^{n}, v_{\theta_{n}}^{n}\right)}^{\prime}\left(\delta u^{n}, \delta v^{n}\right) \\
= & \left(\left(1-S_{\theta_{n}}\right)\left(u^{n}-u^{s}\right)\right) \partial_{x}\left(\delta u^{n}\right)+\delta u^{n} \partial_{x}\left(\left(1-S_{\theta_{n}}\right)\left(u^{n}-u^{s}\right)\right) \\
& +\delta v^{n} \partial_{y}\left(\left(1-S_{\theta_{n}}\right)\left(u^{n}-u^{s}\right)\right)+\left(\left(1-S_{\theta_{n}}\right) v^{n}\right) \partial_{y}\left(\delta u^{n}\right)
\end{aligned}
$$

is the error coming from mollifying the coefficients.

From (4.11), we have

$$
\mathcal{P}\left(u^{n+1}, v^{n+1}\right)=\sum_{j=0}^{n}\left(\mathcal{P}_{\left(S_{\theta_{j}} u^{j}, S_{\theta_{j}} v^{j}\right)}^{\prime}\left(\delta u^{j}, \delta v^{j}\right)+e_{j}\right)+f^{a},
$$

with

$$
f^{a}=\mathcal{P}\left(u^{0}, v^{0}\right):=\partial_{t} u^{0}+u^{0} \partial_{x} u^{0}+v^{0} \partial_{y} u^{0}-\partial_{y}^{2} u^{0} .
$$

Note that if the approximate solution $\left(u^{n}, v^{n}\right)$ converges to a solution of the problem (1.2), then the right hand side in equation (4.12) should go to zero when $n \rightarrow+\infty$. Thus, it is natural to require that $\left(\delta u^{n}, \delta v^{n}\right)$ satisfies the following equation for all $n \geq 0$ :

$$
\mathcal{P}_{\left(u_{\theta_{n}}^{n}, v_{\theta_{n}}^{n}\right)}^{\prime}\left(\delta u^{n}, \delta v^{n}\right)=f^{n}
$$

where $f^{n}$ is defined by

$$
\sum_{j=0}^{n} f^{j}=-S_{\theta_{n}}\left(\sum_{j=0}^{n-1} e_{j}\right)-S_{\theta_{n}} f^{a}
$$

by induction on $n$. Obviously, we have

$$
\left\{\begin{array}{l}
f^{0}=-S_{\theta_{0}} f^{a}, \quad f^{1}=\left(S_{\theta_{0}}-S_{\theta_{1}}\right) f^{a}+S_{\theta_{1}} e_{0} \\
f^{n}=\left(S_{\theta_{n-1}}-S_{\theta_{n}}\right)\left(\sum_{j=0}^{n-2} e_{j}\right)-S_{\theta_{n}} e_{n-1}+\left(S_{\theta_{n-1}}-S_{\theta_{n}}\right) f^{a}, \quad \forall n \geq 2
\end{array}\right.
$$

\section{Existence of the ClAssical SOLUTiOns}

In this section, we study the iteration scheme (4.9) and (4.10) with $f^{n}$ being given in (4.13), by using the estimate (3.4) given in Theorem 3.1. To do this, let us first state the main assumption (MA) on the initial data $\tilde{u}_{0}(x, y)$ of (4.7) as follows:

(MA) For any fixed integers $\tilde{k} \geq 7, k_{0} \geq \tilde{k}+2$, and a real number $\ell>\frac{1}{2}$, suppose that $\tilde{u}_{0} \in \mathcal{A}^{2 k_{0}+1}\left(\mathbb{R}_{+}^{2}\right)$ satisfies the compatibility conditions for the problem (4.7) up to order $k_{0}$, and

$$
\left\|\tilde{u}_{0}\right\|_{\mathcal{A}_{\ell}^{2 k_{0}+1}\left(\mathbb{R}_{+}^{2}\right)}+\left\|\frac{\partial_{y} \tilde{u}_{0}}{\partial_{y} u_{0}^{s}}\right\|_{\mathcal{A}_{\ell}^{2 k_{0}+1}\left(\mathbb{R}_{+}^{2}\right)} \leq \epsilon,
$$

for a small quantity $\epsilon>0$ depending on the norms of $u_{0}^{s}(y)$. 
5.1. The zeroth order approximation. Let us construct the zero-th order approximate solution $\left(u^{0}, v^{0}\right)$ satisfying (4.8) to the problem (1.2). As mentioned in Section 4.2, from equation (4.7) one can easily obtain $\tilde{u}_{0}^{j}(x, y)=\partial_{t}^{j} \tilde{u}(0, x, y)$ and $v_{0}^{j}(x, y)=\partial_{t}^{j} v(0, x, y)$ in terms of $\tilde{u}_{0}(x, y)$ for all $0 \leq j \leq k_{0}$, and then have the following relations:

$$
\left\{\begin{array}{l}
u_{0}^{j}(x, y)=\partial_{y}^{2} u_{0}^{j-1}-\sum_{k=0}^{j-1} C_{j-1}^{k}\left(u_{0}^{k} \partial_{x} u_{0}^{j-1-k}+v_{0}^{k} \partial_{y} u_{0}^{j-1-k}\right), \\
v_{0}^{j}(x, y)=-\int_{0}^{y} \partial_{x} u_{0}^{j}(x, \xi) d \xi
\end{array}\right.
$$

by induction on $j$, with $u_{0}^{j}(x, y)=\tilde{u}_{0}^{j}(x, y)+\left(\partial_{t}^{j} u^{s}\right)(0, y)$.

To construct $\left(\tilde{u}^{0}, v^{0}\right)$ satisfying

$$
\left.\partial_{t}^{j} \tilde{u}^{0}\right|_{t=0}=\tilde{u}_{0}^{j}(x, y),\left.\quad \partial_{t}^{j} v^{0}\right|_{t=0}=v_{0}^{j}(x, y), \quad 0 \leq j \leq k_{0},
$$

we can simply define

$$
\tilde{u}^{0}(t, x, y)=\sum_{j=0}^{k_{0}} \frac{t^{j}}{j !} \tilde{u}_{0}^{j}(x, y), \quad v^{0}(t, x, y)=\sum_{j=0}^{k_{0}} \frac{t^{j}}{j !} v_{0}^{j}(x, y) .
$$

For this approximate solution, we have the following lemma.

Lemma 5.1. Under the assumption (MA), for any fixed $T>0$, there is a constant $C=C\left(k_{0}, T\right)$ that depends only on $k_{0}$ and $T$ such that

$$
\left\|\tilde{u}^{0}\right\|_{\tilde{\mathcal{A}}_{\ell}^{k_{0}+1}\left([0, T] \times \mathbb{R}_{+}^{2}\right)} \leq C \epsilon, \quad\left\|v^{0}\right\|_{\mathcal{D}^{k_{0}}\left([0, T] \times \mathbb{R}_{+}^{2}\right)} \leq C \epsilon,
$$

and

$$
\left\|f^{a}\right\|_{\tilde{\mathcal{A}}_{\ell}^{k_{0}}\left([0, T] \times \mathbb{R}_{+}^{2}\right)} \leq C \epsilon .
$$

Here, we have used the notations

$$
\left\|\tilde{u}^{0}\right\|_{\tilde{\mathcal{A}}_{\ell}^{k}\left([0, T] \times \mathbb{R}_{+}^{2}\right)}:=\sum_{j=0}^{k}\left\|\tilde{u}^{0}\right\|_{W^{j, \infty}\left(0, T ; \mathcal{A}_{\ell}^{k-j}\left(\mathbb{R}_{+}^{2}\right)\right)}
$$

and

$$
f^{a}=\partial_{t} \tilde{u}^{0}+\left(\tilde{u}^{0}+u^{s}\right) \partial_{x} \tilde{u}^{0}+v^{0} \partial_{y}\left(\tilde{u}^{0}+u^{s}\right)-\partial_{y}^{2} \tilde{u}^{0} .
$$

Proof. Since $\tilde{u}_{0} \in \mathcal{A}_{\ell}^{2 k_{0}+1}\left(\mathbb{R}_{+}^{2}\right)$, it follows immediately that

$$
v_{0}(x, y)=-\int_{0}^{y} \partial_{x} \tilde{u}_{0}(x, \eta) d \eta \in \mathcal{D}^{2 k_{0}}\left(\mathbb{R}_{+}^{2}\right),
$$

which implies

$$
\tilde{u}_{0}^{1}=-\left(\tilde{u}_{0}+u_{0}^{s}\right) \partial_{x} \tilde{u}_{0}-v_{0} \partial_{y}\left(\tilde{u}_{0}+u_{0}^{s}\right)+\partial_{y}^{2} \tilde{u}_{0} \in \mathcal{A}_{\ell}^{2 k_{0}}\left(\mathbb{R}_{+}^{2}\right),
$$

and

$$
v_{0}^{1}(x, y)=-\int_{0}^{y} \partial_{x} \tilde{u}_{0}^{1}(x, \eta) d \eta \in \mathcal{D}^{2 k_{0}-1}\left(\mathbb{R}_{+}^{2}\right) .
$$

In this way, using (5.1) and by induction on $j$ we can deduce

$$
\tilde{u}_{0}^{j} \in \mathcal{A}_{\ell}^{2 k_{0}+1-j}\left(\mathbb{R}_{+}^{2}\right), \quad v_{0}^{j} \in \mathcal{D}^{2 k_{0}-j}\left(\mathbb{R}_{+}^{2}\right),
$$

for all $j \leq k_{0}$, and

$$
\left\|\tilde{u}_{0}^{j}\right\|_{\mathcal{A}_{\ell}^{2 k_{0}-j+1}\left(\mathbb{R}_{+}^{2}\right)} \leq C(j) \epsilon
$$


for a constant $C(j)$ depending only on $j$. Then, (5.3) and (5.4) follow immediately from the construction (5.2).

Remark 5.2. Denoting $u^{0}=\tilde{u}^{0}+u^{s}$, it is easy to see that $\left(u^{0}, v^{0}\right)$ is an approximate solution to the original problem (1.2) satisfying

$$
\left\{\begin{array}{l}
u_{t}^{0}+u^{0} u_{x}^{0}+v^{0} u_{y}^{0}-u_{y y}^{0}=f^{a}, \quad t>0, \quad(x, y) \in \mathbb{R}_{+}^{2} \\
\partial_{x} u^{0}+\partial_{y} v^{0}=0, \quad(x, y) \in \mathbb{R}_{+}^{2}, t \geq 0 \\
\left.\left(u^{0}, v^{0}\right)\right|_{y=0}=0, \quad \lim _{y \rightarrow+\infty} u^{0}=1 \\
\left.u^{0}\right|_{t=0}=u_{0}(x, y)
\end{array}\right.
$$

with $\left.\partial_{t}^{j} f^{a}\right|_{t=0}=0$ for all $0 \leq j \leq k_{0}-1$.

Lemma 5.3. Under the assumptions (MA), for any fixed $T>0$, there is a constant $C(k, T)$ such that,

$$
\left\|\frac{\partial_{y} \tilde{u}^{0}}{\partial_{y} u^{s}}\right\|_{\mathcal{A}_{\ell}^{k_{0}+1}\left([0, T] \times \mathbb{R}_{+}^{2}\right)}+\left\|\frac{f^{a}}{\partial_{y} u^{s}}\right\|_{\mathcal{A}_{\ell}^{k_{0}\left([0, T] \times \mathbb{R}_{+}^{2}\right)}} \leq C \epsilon .
$$

This result can be proved in a way similar to the proof of Lemma 5.1, so we omit it for brevity.

Remark 5.4. From the smallness of the first term given in (5.6), it is easy to see that for a fixed $T>0$, there is a small $\epsilon$ such that when the conditions of Lemmas 5.1 and 5.3 hold, we have the monotonicity

$$
\partial_{y} u^{0}(t, x, y)>0, \quad(t, x, y) \in[0, T] \times \mathbb{R}_{+}^{2} .
$$

5.2. Estimates of the approximate solutions. Obviously, the problem (4.10) can be written as

$$
\left\{\begin{array}{l}
\partial_{t}\left(\delta u^{n}\right)+u_{\theta_{n}}^{n} \partial_{x}\left(\delta u^{n}\right)+v_{\theta_{n}}^{n} \partial_{y}\left(\delta u^{n}\right)+\delta u^{n} \partial_{x}\left(u_{\theta_{n}}^{n}\right)+\delta v^{n} \partial_{y}\left(u_{\theta_{n}}^{n}\right)-\partial_{y}^{2}\left(\delta u^{n}\right)=f^{n} \\
\partial_{x}\left(\delta u^{n}\right)+\partial_{y}\left(\delta v^{n}\right)=0, \\
\left.\delta u^{n}\right|_{y=0}=\left.\delta v^{n}\right|_{y=0}=0, \quad \lim _{y \rightarrow+\infty} \delta u^{n}=0 \\
\left.\delta u^{n}\right|_{t=0}=0
\end{array}\right.
$$

where

$$
u_{\theta_{n}}^{n}=u^{s}+S_{\theta_{n}}\left(\tilde{u}^{0}+\sum_{0 \leq j \leq n-1} \delta u^{j}\right), \quad v_{\theta_{n}}^{n}=S_{\theta_{n}}\left(v^{0}+\sum_{0 \leq j \leq n-1} \delta v^{j}\right) .
$$

Set

$$
w^{n}=\partial_{y}\left(\frac{\delta u^{n}}{\partial_{y} u_{\theta_{n}}^{n}}\right) .
$$

As in Section 3, from (5.7), we know that $w^{n}$ satisfies

$$
\left\{\begin{array}{l}
\partial_{t} w^{n}+\partial_{x}\left(u_{\theta_{n}}^{n} w^{n}\right)+\partial_{y}\left(v_{\theta_{n}}^{n} w^{n}\right)-2 \partial_{y}\left(\eta^{n} w^{n}\right) \\
\quad+\partial_{y}\left(\zeta^{n} \int_{0}^{y} w^{n}(t, x, \tilde{y}) d \tilde{y}\right)-\partial_{y}^{2} w^{n}=\partial_{y} \tilde{f}^{n} \\
\left.\left(\partial_{y} w^{n}+2 \eta^{n} w^{n}\right)\right|_{y=0}=-\left.\tilde{f}^{n}\right|_{y=0} \\
\left.w^{n}\right|_{t=0}=0
\end{array}\right.
$$


where

$$
\eta^{n}=\frac{\partial_{y}^{2} u_{\theta_{n}}^{n}}{\partial_{y} u_{\theta_{n}}^{n}}, \quad \zeta^{n}=\frac{\left(\partial_{t}+u_{\theta_{n}}^{n} \partial_{x}+v_{\theta_{n}}^{n} \partial_{y}-\partial_{y}^{2}\right) \partial_{y} u_{\theta_{n}}^{n}}{\partial_{y} u_{\theta_{n}}^{n}}
$$

and

$$
\tilde{f}^{n}=\frac{f^{n}}{\partial_{y} u_{\theta_{n}}^{n}}=\frac{\left(S_{\theta_{n-1}}-S_{\theta_{n}}\right)\left(\sum_{j=0}^{n-2} e_{j}\right)-S_{\theta_{n}} e_{n-1}+\left(S_{\theta_{n-1}}-S_{\theta_{n}}\right) f^{a}}{\partial_{y} u_{\theta_{n}}^{n}} .
$$

From the main assumption (MA) given at the beginning of this section, and the construction of the approximate solution $\left(\tilde{u}^{0}, v^{0}\right)$ to the problem (4.7), it is easy to show by induction on $n$ that the compatibility conditions for the problem (5.8) up to order $k_{0}$ hold for all $n \geq 0$.

Similar to Section 3 , set

$$
\begin{aligned}
\lambda_{k_{1}, k_{2}}^{n}= & \left\|u_{\theta_{n}}^{n}-u^{s}\right\|_{\mathcal{B}_{0,0}^{k_{1}, k_{2}}}+\left\|\partial_{\mathcal{T}}^{k_{1}} \partial_{y}^{k_{2}} u^{s}\right\|_{L_{y}^{2}\left(L_{t}^{\infty}\right)}+\left\|\partial_{\mathcal{T}}^{k_{1}} \partial_{y}^{k_{2}} v_{\theta_{n}}^{n}\right\|_{L_{y}^{\infty}\left(L_{t, x}^{2}\right)} \\
& +\left\|\partial_{\mathcal{T}}^{k_{1}} \partial_{y}^{k_{2}} \bar{\eta}^{n}\right\|_{L_{y}^{2}\left(L_{t, x}^{\infty}\right)}+\left\|\eta^{n}-\bar{\eta}^{n}\right\|_{\mathcal{B}_{0,0}^{k_{1}, k_{2}}}+\left\|\zeta^{n}\right\|_{\mathcal{B}_{0, l}^{k_{1}, k_{2}}},
\end{aligned}
$$

with $\bar{\eta}^{n}=\frac{\partial_{y}^{2} u^{s}}{\partial_{y} u_{\theta_{n}}^{n}}$, and

$$
\lambda_{k}^{n}=\sum_{k_{1}+\left[\frac{k_{2}+1}{2}\right] \leq k} \lambda_{k_{1}, k_{2}}^{n}
$$

That is,

$$
\lambda_{k}^{n}=\left\|u_{\theta_{n}}^{n}-u^{s}\right\|_{\mathcal{A}_{0}^{k}}+\left\|u^{s}\right\|_{\mathcal{C}_{0}^{k}}+\left\|v_{\theta_{n}}^{n}\right\|_{\mathcal{D}_{0}^{k}}+\left\|\bar{\eta}^{n}\right\|_{\mathcal{C}_{0}^{k}}+\left\|\eta^{n}-\bar{\eta}^{n}\right\|_{\mathcal{A}_{0}^{k}}+\left\|\zeta^{n}\right\|_{\mathcal{A}_{l}^{k}} .
$$

By applying Theorem 3.1 to the problem (5.8), we have the following proposition.

Proposition 5.5. Under the assumption (MA), the solution $w^{n}$ to the problem (5.8) satisfies

$$
\left\|w^{n}\right\|_{\mathcal{A}_{\ell}^{k}} \leq C_{1}\left(\lambda_{3}^{n}\right)\left\|\tilde{f}^{n}\right\|_{\mathcal{A}_{\ell}^{k}}+C_{2}\left(\lambda_{3}^{n}\right) \lambda_{k}^{n}\left\|\tilde{f}^{n}\right\|_{\mathcal{A}_{\ell}^{3}},
$$

where $C_{1}\left(\lambda_{3}^{n}\right), C_{2}\left(\lambda_{3}^{n}\right)$ are polynomials of $\lambda_{3}^{n}$ of order less than or equal to $k$.

The key step in proving the convergence of the Nash-Moser-Hörmander iteration scheme (4.9) and (4.10) is given by the following result.

Theorem 5.6. Under the assumption (MA), there exists a positive constant $C_{0}$, such that

$$
\left\|w^{n}\right\|_{\mathcal{A}_{\ell}^{k}} \leq C_{0} \epsilon \theta_{n}^{\max \{3-\tilde{k}, k-\tilde{k}\}} \Delta \theta_{n}
$$

holds for all $n \geq 0,0 \leq k \leq k_{0}$ where $\theta_{n}=\sqrt{\theta_{0}^{2}+n}$ and $\Delta \theta_{n}=\theta_{n+1}-\theta_{n}$.

Theorem 5.6 will be proved by induction on $n$. First of all, to apply Proposition [5.5. we need to estimate $\lambda_{k}^{n}$ and $\tilde{f}^{n}$ by induction on $n$ also. For this purpose, we first give the following estimates, some of the proofs being postponed to Section 7 The proof of Theorem 5.6 will be completed at the end of this subsection. 
Lemma 5.7. Suppose that the assumption (MA) and (5.12) for $w^{j}, 0 \leq j \leq n-1$, hold. Then there is a constant $C_{1}>0$, such that

$$
\begin{aligned}
\left\|\delta u^{j}\right\|_{\mathcal{A}_{\ell}^{k}} & \leq C_{1} \epsilon \theta_{j}^{\max \{3-\tilde{k}, k-\tilde{k}\}} \Delta \theta_{j}, \quad 0 \leq k \leq k_{0}, \\
\left\|\frac{\delta u^{j}}{\partial_{y} u^{s}}\right\|_{L^{\infty}\left([0, T] \times \mathbb{R}_{+}^{2}\right)} & \leq C_{1} \epsilon \theta_{j}^{3-\tilde{k}} \Delta \theta_{j},
\end{aligned}
$$

and

$$
\left\|\frac{\delta u^{j}}{\partial_{y} u^{s}}\right\|_{\mathcal{D}_{0}^{k-1}} \leq C_{1} \epsilon \theta_{j}^{\max \{3-\tilde{k}, k-1-\tilde{k}\}} \Delta \theta_{j}, \quad 1 \leq k \leq k_{0},
$$

hold for all $0 \leq j \leq n-1$.

Since the proof of this lemma is technical, it will be given in Section 7

As $\delta v^{j}(t, x, y)=-\int_{0}^{y}\left(\partial_{x} \delta u^{j}\right)(t, x, \tilde{y}) d \tilde{y}$, from (5.13) we immediately have the following estimate

Lemma 5.8. Under the same assumptions as for Lemma 5.7, there is a constant $C_{2}>0$, such that

$$
\left\|\delta v^{j}\right\|_{\mathcal{D}_{0}^{k}} \leq C_{2} \epsilon \theta_{j}^{\max \{3-\tilde{k}, k+1-\tilde{k}\}} \Delta \theta_{j}, \quad 0 \leq k \leq k_{0}-1,
$$

holds for all $0 \leq j \leq n-1$.

Based on Lemma 5.7 and Lemma 5.8 we have the following result:

Lemma 5.9. Under the same assumptions as for Lemma 5.7, there is a constant $C_{3}>0$, such that

$$
\begin{gathered}
\left\|u^{n}-u^{s}\right\|_{\mathcal{A}_{l}^{k}} \leq C_{3} \epsilon \theta_{n}^{\max \{0, k+1-\tilde{k}\}}, \quad 0 \leq k \leq k_{0}, \\
\left\|v_{\theta_{n}}^{n}\right\|_{L^{\infty}\left([0, T] \times \mathbb{R}_{+}^{2}\right)}+\left\|v_{\theta_{n}}^{n}\right\|_{L^{\infty}\left([0, \infty)_{y}, L^{2}\left([0, T] \times \mathbb{R}_{x}\right)\right.} \leq C_{3},
\end{gathered}
$$

and

$$
\left\|v_{\theta_{n}}^{n}\right\|_{\mathcal{D}_{0}^{k}} \leq\left\{\begin{array}{l}
C_{\rho}\left\|v^{n}\right\|_{\mathcal{D}_{0}^{k}} \leq C_{3} \epsilon \theta_{n}^{\max \{0, k+2-\tilde{k}\}}, \quad 0 \leq k \leq k_{0}-1, \\
C_{\rho} \theta_{n}\left\|v^{n}\right\|_{\mathcal{D}_{0}^{k_{0}-1}} \leq C_{3} \epsilon \theta_{n}^{\max \left\{1, k_{0}+2-\tilde{k}\right\}}, \quad k=k_{0},
\end{array}\right.
$$

hold, where $C_{\rho}>0$ is given in (4.1).

Proof. From the identity

$$
u^{n}-u^{s}=\tilde{u}^{0}+\sum_{j=0}^{n-1} \delta u^{j},
$$

we have immediately by using (5.3) and Lemma 5.7 that

$$
\begin{aligned}
\left\|u^{n}-u^{s}\right\|_{\mathcal{A}_{l}^{k}} & \leq\left\|\tilde{u}^{0}\right\|_{\mathcal{A}_{l}^{k}}+\sum_{j=0}^{n-1}\left\|\delta u^{j}\right\|_{\mathcal{A}_{l}^{k}} \\
& \leq C^{a} \epsilon+C_{1} \epsilon \sum_{j=0}^{n-1} \theta_{j}^{\max \{3-\tilde{k}, k-\tilde{k}\}} \Delta \theta_{j} \\
& \leq C^{a} \epsilon+C_{1} \tilde{C} \epsilon \theta_{n}^{\max \{0, k+1-\tilde{k}\}} .
\end{aligned}
$$


Here, we have used the fact that

$$
\sum_{p=0}^{j-1} \theta_{p}^{k-\tilde{k}} \Delta \theta_{p} \leq\left\{\begin{array}{l}
\tilde{C} \theta_{j}^{k+1-\tilde{k}}, \quad \text { as } k-\tilde{k} \geq 0, \\
\tilde{C}, \quad \text { as } k-\tilde{k} \leq-2,
\end{array}\right.
$$

for an absolute constant $\tilde{C}$.

From (5.20), we obtain the estimate (5.17) immediately. Similarly, from the identity

$$
v^{n}=v^{0}+\sum_{j=0}^{n-1} \delta v^{j},
$$

we can easily deduce the estimates (5.18) and (5.19) by using Lemma 5.8 .

As a direct consequence of the estimate (5.17), there is a constant $\tilde{C}_{3}>0$ such that

$$
\left\|u_{\theta_{n}}^{n}-u^{s}\right\|_{\mathcal{A}_{\ell}^{k}} \leq \tilde{C}_{3} \epsilon \theta_{n}^{\max \{0, k+1-\tilde{k}\}}, \quad 0 \leq k \leq k_{0} .
$$

To get the estimate of $\lambda_{k}^{n}$, we need to estimate the norms of $\eta^{n}=\frac{\partial_{y}^{2} u_{\theta_{n}}^{n}}{\partial_{y} u_{\theta_{n}}^{n}}$ and

$$
\zeta^{n}=\frac{\left(\partial_{t}+u_{\theta_{n}}^{n} \partial_{x}+v_{\theta_{n}}^{n} \partial_{y}-\partial_{y}^{2}\right) \partial_{y} u_{\theta_{n}}^{n}}{\partial_{y} u_{\theta_{n}}^{n}},
$$

which are given as follows. Again, the proofs of the next two lemmas will be given in Section 7.

Lemma 5.10. Under the same assumptions as for Lemma [5.7, there is a constant $C_{4}>0$, such that

$$
\left\|\eta^{n}-\bar{\eta}^{n}\right\|_{\mathcal{A}_{\ell}^{k}} \leq\left\{\begin{array}{l}
C_{4} \epsilon \theta_{n}^{\max \{1, k+2-\tilde{k}\}}, \quad 4 \leq k \leq k_{0}, \\
C_{4} \epsilon, \quad k=3,
\end{array}\right.
$$

and

$$
\left\|\bar{\eta}^{n}\right\|_{\mathcal{C}_{\ell}^{k}} \leq C_{4}\left(1+\epsilon \theta_{n}^{\max \{0, k+3-\tilde{k}\}}\right), \quad 0 \leq k \leq k_{0},
$$

where $\bar{\eta}^{n}=\frac{\partial_{y}^{2} u^{s}}{\partial_{y} u_{\theta_{n}}^{n}}$.

Lemma 5.11. Under the same assumptions as for Lemma 5.7, for $\zeta^{n}$ defined in (5.23), there is a positive constant $C_{5}$ such that

$$
\left\|\zeta^{n}\right\|_{\mathcal{A}_{\ell}^{k}} \leq\left\{\begin{array}{l}
C_{5} \theta_{n}^{\max \{1, k+3-\tilde{k}\}}, \quad 4 \leq k \leq k_{0}, \\
C_{5}, \quad k=3 .
\end{array}\right.
$$

By plugging the estimates (5.19), (5.24), (5.25), (5.26) and (5.22) into the definition (5.10) of $\lambda_{k}^{n}$, we conclude the following result.

Proposition 5.12. Suppose that the assumption (MA) and (5.12) for $w^{j}, 0 \leq j \leq$ $n-1$, hold. There exists a positive constant $C_{6}>0$ depending on $C_{p}(1 \leq p \leq 5)$ given in Lemmas [5.7 [5.11, such that

$$
\lambda_{k}^{n} \leq\left\{\begin{array}{l}
C_{6} \theta_{n}^{\max \{1, k+3-\tilde{k}\}}, \quad 4 \leq k \leq k_{0}, \\
C_{6}, \quad k=3 .
\end{array}\right.
$$


To estimate $\tilde{f}^{n}$ defined in (5.9), we will need the following two estimates whose proofs will be given in Section 7

Lemma 5.13. Under the same assumptions as for Lemma 5.7, there is a constant $C_{7}>0$, such that

$$
\left\|\left(\frac{\partial_{y} u_{\theta_{n}}^{n}}{\partial_{y} u^{s}}\right)^{-1}\right\|_{L^{\infty}} \leq 2, \quad\left\|\left(\frac{\partial_{y} u_{\theta_{n}}^{n}}{\partial_{y} u^{s}}\right)^{-1}\right\|_{\dot{\mathcal{A}}_{0}^{k}} \leq C_{7} \epsilon \theta_{n}^{\max \{0, k+1-\tilde{k}\}},
$$

hold, with $1 \leq k \leq k_{0}$.

Lemma 5.14. Under the same assumptions as for Lemma [5.7, there is a constant $C_{8}>0$, such that for the error terms $e_{j}^{(1)}=\delta u^{j} \partial_{x} \delta u^{j}+\delta v^{j} \partial_{y} \delta u^{j}$ and

$$
\begin{aligned}
e_{j}^{(2)}= & \left(\left(1-S_{\theta_{j}}\right)\left(u^{j}-u^{s}\right)\right) \partial_{x}\left(\delta u^{j}\right)+\delta u^{j} \partial_{x}\left(\left(1-S_{\theta_{j}}\right)\left(u^{j}-u^{s}\right)\right) \\
& +\delta v^{j} \partial_{y}\left(\left(1-S_{\theta_{j}}\right)\left(u^{j}-u^{s}\right)\right)+\left(\left(1-S_{\theta_{n}}\right) v^{j}\right) \partial_{y}\left(\delta u^{j}\right),
\end{aligned}
$$

the following estimates,

$$
\left\|\frac{e_{j}^{(1)}}{\partial_{y} u^{s}}\right\|_{\mathcal{A}_{\ell}^{k_{1}}} \leq C_{8} \epsilon^{2} \theta_{j}^{\max \left\{6-2 \tilde{k}, k_{1}+3-2 \tilde{k}\right\}} \Delta \theta_{j}
$$

and

$$
\left\|\frac{e_{j}^{(2)}}{\partial_{y} u^{s}}\right\|_{\mathcal{A}_{l}^{k_{1}}} \leq C_{8} \epsilon^{2} \theta_{j}^{\max \left(3-\tilde{k}, k_{1}+5-2 \tilde{k}\right)} \Delta \theta_{j}
$$

hold for all $k_{1} \leq k_{0}-1$ and $0 \leq j \leq n-1$.

In summary, for

$$
\tilde{f}^{n}=\frac{f^{n}}{\partial_{y} u_{\theta_{n}}^{n}}=\frac{\left(S_{\theta_{n-1}}-S_{\theta_{n}}\right)\left(\sum_{j=0}^{n-2} e_{j}\right)-S_{\theta_{n}} e_{n-1}+\left(S_{\theta_{n-1}}-S_{\theta_{n}}\right) f^{a}}{\partial_{y} u_{\theta_{n}}^{n}},
$$

with $e_{n}=e_{n}^{(1)}+e_{n}^{(2)}$, we have Proposition 5.15.

Proposition 5.15. Suppose that the assumption (MA) and (5.12) for $w^{j}, 0 \leq j \leq$ $n-1$, hold, then there exists a constant $C_{9}>0$ such that

$$
\left\|\tilde{f}^{n}\right\|_{\mathcal{A}_{\ell}^{k}} \leq C_{9} \epsilon \theta_{n}^{\max \{3-\tilde{k}, k-\tilde{k}\}} \Delta \theta_{n}, \quad 0 \leq k \leq k_{0} .
$$

Proof. From $\tilde{f}^{n}=\frac{f^{n}}{\partial_{y} u_{\theta_{n}}^{n}}=\frac{f^{n}}{\partial_{y} u^{s}}\left(\frac{\partial_{y} u_{\theta_{n}}^{n}}{\partial_{y} u^{s}}\right)^{-1}$, by using Lemma 2.1 and Lemma 5.13 we have

$$
\left\|\tilde{f}^{n}\right\|_{\mathcal{A}_{\ell}^{k}} \leq M_{k}\left\{2\left\|\frac{f^{n}}{\partial_{y} u^{s}}\right\|_{\mathcal{A}_{\ell}^{k}}+\left\|\frac{f^{n}}{\partial_{y} u^{s}}\right\|_{L_{\ell}^{\infty}} C_{7} \epsilon \theta_{n}^{\max \{0, k+1-\tilde{k}\}}\right\} .
$$

On the other hand, using (4.1) and (4.2), for any $k, k_{j} \geq 0(j=1,2,3)$, we have

$$
\begin{aligned}
\left\|\frac{f^{n}}{\partial_{y} u^{s}}\right\|_{\mathcal{A}_{\ell}^{k}} \leq & C_{\rho}\left\{\sum_{j=0}^{n-2}\left\|\frac{e_{j}}{\partial_{y} u^{s}}\right\|_{\mathcal{A}_{\ell}^{k_{1}}} \theta_{n}^{k-k_{1}} \Delta \theta_{n}+\left\|\frac{e_{n-1}}{\partial_{y} u^{s}}\right\|_{\mathcal{A}_{\ell}^{k_{2}}} \theta_{n}^{\left(k-k_{2}\right)_{+}}\right. \\
& \left.+\left\|\frac{f^{a}}{\partial_{y} u^{s}}\right\| \|_{\mathcal{A}_{\ell}^{k_{3}}} \theta_{n}^{k-k_{3}} \Delta \theta_{n}\right\} .
\end{aligned}
$$


Thus, by using (5.28), (5.29) in (5.31), we get

$$
\begin{aligned}
\left\|\frac{f^{n}}{\partial_{y} u^{s}}\right\|_{\mathcal{A}_{\ell}^{k}} \leq & C_{\rho}\left\{2 C_{8} \sum_{j=0}^{n-2} \epsilon^{2} \theta_{j}^{\max \left\{3-\tilde{k}, k_{1}+5-2 \tilde{k}\right\}} \Delta \theta_{j} \theta_{n}^{k-k_{1}} \Delta \theta_{n}\right. \\
& \left.+2 C_{8} \epsilon^{2} \theta_{n-1}^{\max \left\{3-\tilde{k}, k_{2}+5-2 \tilde{k}\right\}} \Delta \theta_{n-1} \theta_{n}^{\left(k-k_{2}\right)_{+}}+C^{a} \epsilon \theta_{n}^{k-k_{3}} \Delta \theta_{n}\right\},
\end{aligned}
$$

for $k_{1} \leq k_{0}-1$ and $k_{2} \leq k_{0}-1$, provided $\left\|\frac{f^{a}}{\partial_{y} u^{s}}\right\|_{\mathcal{A}_{l}^{k_{3}}} \leq C^{a} \epsilon$.

When $k=3$, by setting $k_{1}=k_{3}=\tilde{k}$ and $k_{2}=3$ in (5.32) we get

$$
\left\|\frac{f^{n}}{\partial_{y} u^{s}}\right\|_{\mathcal{A}_{\ell}^{3}} \leq C_{\rho}\left(2 C_{8}(1+\tilde{C}) \epsilon^{2}+C^{a} \epsilon\right) \theta_{n}^{3-\tilde{k}} \Delta \theta_{n} .
$$

When $4 \leq k \leq k_{0}$, by choosing $k_{1} \geq 1+\tilde{k}, k_{2}=\tilde{k}-2$ and $k_{3}=\tilde{k}$ in (5.32) we obtain

$$
\left\|\frac{f^{n}}{\partial_{y} u^{s}}\right\|_{\mathcal{A}_{\ell}^{k}} \leq C_{\rho}\left(2 C_{8}(1+\tilde{C}) \epsilon^{2}+C^{a} \epsilon\right) \theta_{n}^{k-\tilde{k}} \Delta \theta_{n} .
$$

Here, we have used the fact that $\left(k-k_{2}\right)_{+}+k_{2}+5-\tilde{k} \leq k$ for all $4 \leq k \leq k_{0}$.

Combining (5.33) with (5.34), we conclude the estimate (5.30).

\section{Proof of Theorem 5.6:}

We are now ready to conclude the proof of Theorem [5.6 by induction on $n$.

For $n=0$, from the assumption (MA), Lemma 5.1, and Lemma 5.5, we get immediately that for any fixed $T>0$, there is a constant $C^{a}=C^{a}\left(k_{0}, T\right)$ such that

$$
\left\|\tilde{u}^{0}\right\|_{\mathcal{A}_{\ell}^{k_{0}+1}}+\left\|\frac{\partial_{y} \tilde{u}^{0}}{\partial_{y} u^{s}}\right\|_{\mathcal{A}_{\ell}^{k_{0}+1}}+\left\|\frac{f^{a}}{\partial_{y} u^{s}}\right\|_{\mathcal{A}_{\ell}^{k_{0}}} \leq C^{a} \epsilon .
$$

This implies that $\tilde{f}^{0}=\frac{f^{a}}{\partial_{y}\left(u^{s}+S_{\theta_{0}} \tilde{u}^{0}\right)}$ satisfies

$$
\left\|\tilde{f}^{0}\right\|_{\mathcal{A}_{\ell}^{k_{0}}} \leq \tilde{C}^{a} \epsilon
$$

for a constant $\tilde{C}^{a}$

A direct calculation yields

$$
\lambda_{k}^{0} \leq\left\|u^{s}\right\|_{\mathcal{C}_{0}^{k}}+\left\|\frac{\partial_{y}^{2} u^{s}}{\partial_{y} u^{s}}\right\|_{\mathcal{C}_{0}^{k}}+C_{1}{ }^{a} \epsilon \leq C_{k_{0}}, \quad \forall k \leq k_{0},
$$

for a constant $C_{1}{ }^{a}$ depending on $C^{a}$ and $\tilde{C}^{a}$ given above.

By applying Proposition 5.5 for $w^{0}$, and using the above estimates, it follows that

$$
\left\|w^{0}\right\|_{\mathcal{A}_{\ell}^{k}} \leq \bar{C}_{k_{0}} \epsilon, \quad \forall k \leq k_{0},
$$

for a constant $\bar{C}_{k_{0}}$ depending on $\tilde{C}^{a}$ and $C_{k_{0}}$ given above. Hence, the estimate (5.12) for the case $n=0$ follows immediately from (5.35) with a constant $C_{0}$ that depends on $\bar{C}_{k_{0}}, k_{0}, \tilde{k}$, and $\theta_{0}$.

Now, assuming that (5.12) holds for all $w^{j}$ with $0 \leq j \leq n-1$, we are going to prove it for $w^{n}$. In fact, from the estimates (5.11) and (5.30), we get

$$
\left\|w^{n}\right\|_{\mathcal{A}_{\ell}^{k}} \leq C_{1}\left(\lambda_{3}^{n}\right) C_{9} \epsilon \theta_{n}^{\max \{3-\tilde{k}, k-\tilde{k}\}}+C_{2}\left(\lambda_{3}^{n}\right) \lambda_{k}^{n} C_{9} \epsilon \theta_{n}^{3-\tilde{k}},
$$


which implies by Proposition 5.12 that

$$
\left\|w^{n}\right\|_{\mathcal{A}_{\ell}^{3}} \leq C_{0} \epsilon \theta_{n}^{3-\tilde{k}}
$$

and

$$
\left\|w^{n}\right\|_{\mathcal{A}_{\ell}^{k}} \leq C_{0} \epsilon \theta_{n}^{k-\tilde{k}}
$$

for all $4 \leq k \leq k_{0}$, with the constant $C_{0} \geq\left(C_{1}\left(C_{6}\right)+C_{2}\left(C_{6}\right) C_{6}\right) C_{9}$.

5.3. Convergence of the iteration scheme. In this subsection, we will prove the convergence of the iteration scheme, and this immediately yields the existence of classical solutions to the Prandtl equation (1.2).

From the iteration scheme (4.9) and (4.10) with $f^{n}$ defined in (4.13), we know that the approximate solution

$$
u^{n+1}=u^{s}+\tilde{u}^{0}+\sum_{j=0}^{n} \delta u^{j}, \quad v^{n+1}=v^{0}+\sum_{j=0}^{n} \delta v^{j},
$$

satisfies

$$
\left\{\begin{aligned}
& \mathcal{P}\left(u^{n+1}, v^{n+1}\right)=\left(1-S_{\theta_{n}}\right) \sum_{j=0}^{n} e_{j}+S_{\theta_{n}} e_{n} \\
&+\left(1-S_{\theta_{n}}\right) f^{a}, \partial_{x} u^{n+1}+\partial_{y} v^{n+1}=0, \\
&\left.u^{n+1}\right|_{y=0}=\left.v^{n+1}\right|_{y=0}=0, \quad \lim _{y \rightarrow+\infty} u^{n+1}=1 \\
&\left.u^{n+1}\right|_{t=0}=u_{0}(x, y) .
\end{aligned}\right.
$$

From the estimates (5.13) and (5.16), we know that there exist $u \in u^{s}+\mathcal{A}_{\ell}^{\tilde{k}-2}$ and $v \in \mathcal{D}_{0}^{\tilde{k}-3}$, such that

$$
\lim _{n \rightarrow+\infty}\left\|u^{n}-u\right\|_{\mathcal{A}_{\ell}^{\tilde{k}-2}}=0, \quad \lim _{n \rightarrow+\infty}\left\|v^{n}-v\right\|_{\mathcal{D}_{0}^{\tilde{k}-3}}=0 .
$$

To verify that the limit $(u, v)$ is a classical solution to the problem (1.2), it is enough to show that the right hand side of the first equation in (5.36) converges to zero as $n \rightarrow+\infty$.

Obviously, we have

$$
\left\|\left(1-S_{\theta_{n}}\right)\left(f^{a}+\sum_{j=0}^{n} e_{j}\right)\right\|_{\mathcal{A}_{\ell}^{k}} \leq \theta_{n}^{-1}\left(\left\|f^{a}\right\|_{\mathcal{A}_{\ell}^{k+1}}+\sum_{j=0}^{n}\left\|e_{j}\right\|_{\mathcal{A}_{\ell}^{k+1}}\right) .
$$

Thus, it is enough to prove the convergence of series $\sum_{j=0}^{+\infty}\left\|e_{j}\right\|_{\mathcal{A}_{\ell}^{k+1}}$. Recall

$$
e_{j}=e_{j}^{(1)}+e_{j}^{(2)},
$$

with

$$
e_{j}^{(1)}=\delta u^{j} \partial_{x}\left(\delta u^{j}\right)+\delta v^{j} \partial_{y}\left(\delta u^{j}\right)
$$

and

$$
e_{j}^{(2)}=\partial_{y}\left(\delta v^{j}\left(\left(1-S_{\theta_{j}}\right)\left(u^{j}-u^{s}\right)\right)+\left(\left(1-S_{\theta_{j}}\right) v^{j}\right)\left(\delta u^{j}\right)\right) .
$$

By using Lemma 2.1, it follows that

$$
\begin{aligned}
\left\|e_{j}^{(1)}\right\|_{\mathcal{A}_{\ell}^{k+1}} \leq & M_{k}\left(\left\|\delta u^{j}\right\|_{L^{\infty}}\left\|\delta u^{j}\right\|_{\mathcal{A}_{\ell}^{k+2}}+\left\|\delta v^{j}\right\|_{L^{\infty}}\left\|\delta u^{j}\right\|_{\mathcal{A}_{\ell}^{k+2}}\right. \\
& \left.+\left\|\delta v^{j}\right\|_{\mathcal{D}_{0}^{k+2}}\left\|\delta u^{j}\right\|_{L_{y, \ell}^{2}\left(L_{t, x}^{\infty}\right)}\right) \\
\leq & C_{10} \epsilon^{2} \theta_{j}^{k+5-2 \tilde{k}} \Delta \theta_{j},
\end{aligned}
$$


and

$$
\begin{aligned}
\left\|e_{j}^{(2)}\right\|_{\mathcal{A}_{\ell}^{k+1}} \leq & M_{k}\left(\left\|\delta v^{j}\right\|_{L^{\infty}}\left\|u^{j}-u^{s}\right\|_{\mathcal{A}_{\ell}^{k+2}}+\left\|\delta v^{j}\right\|_{\mathcal{D}_{0}^{k+2}}\left\|u^{j}-u^{s}\right\|_{L_{y, \ell}^{2}\left(L_{t, x}^{\infty}\right)}\right. \\
& \left.+\left\|v^{j}\right\|_{L^{\infty}}\left\|\delta u^{j}\right\|_{\mathcal{A}_{\ell}^{k+2}}+\left\|v^{j}\right\|_{\mathcal{D}_{0}^{k+2}}\left\|\delta u^{j}\right\|_{L_{y, \ell}^{2}\left(L_{t, x}^{\infty}\right)}\right) \\
\leq & C_{10} \epsilon^{2} \theta_{j}^{k+3-\tilde{k}} \Delta \theta_{j},
\end{aligned}
$$

for a positive constant $C_{10}>0$, where we have used (5.13), (5.16), and (5.17). Therefore, we obtain

$$
\sum_{j=0}^{+\infty}\left\|e_{j}\right\|_{\mathcal{A}_{\ell}^{k+1}} \leq C \sum_{j=0}^{+\infty} \theta_{j}^{k+3-\tilde{k}} \Delta \theta_{j} \leq C \tilde{C},
$$

for all $k \leq \tilde{k}-5$. And this concludes the convergence of the iteration scheme and the existence of classical solutions to the Prandtl equation (1.2).

\section{UNIQUENESS AND STABILITY}

In this section, we study the stability of classical solutions to the Prandtl equation (1.2), and thus the uniqueness of the classical solution obtained in Section 5 will follow immediately.

Let $\left(u^{1}, v^{1}\right)$ and $\left(u^{2}, v^{2}\right)$ be two classical solutions to the problem (1.2) in the solution spaces given in Theorem 1.1 with the initial data $u_{0}^{1}(x, y)$ and $u_{0}^{2}(x, y)$ as two small perturbations of $u_{0}^{s}(y)$ as stated in Theorem 1.1. Denoting by

$$
u=u^{1}-u^{2}, \quad v=v^{1}-v^{2}, \quad \tilde{u}=\frac{u^{1}+u^{2}}{2}, \quad \tilde{v}=\frac{v^{1}+v^{2}}{2},
$$

then from (1.2), we deduce that $(u, v)$ satisfies

$$
\left\{\begin{array}{l}
\partial_{t} u+\tilde{u} \partial_{x} u+\tilde{v} \partial_{y} u+u \partial_{x} \tilde{u}+v \partial_{y} \tilde{u}-\partial_{y}^{2} u=0, \partial_{x} u+\partial_{y} v=0, \\
\left.u\right|_{y=0}=\left.v\right|_{y=0}=0, \quad \lim _{y \rightarrow+\infty} u=0 \\
\left.u\right|_{t=0}=u_{0}(x, y):=u_{0}^{1}-u_{0}^{2} .
\end{array}\right.
$$

As in Section 3 , set

(6.2) $w(t, x, y)=\left(\frac{u}{\partial_{y} \tilde{u}}\right)_{y}(t, x, y), \quad$ that is, $u(t, x, y)=\left(\partial_{y} \tilde{u}\right) \int_{0}^{y} w\left(t, x, y^{\prime}\right) d y^{\prime}$.

Then, from (6.1) we know that $w(t, x, y)$ satisfies

$$
\left\{\begin{array}{l}
\partial_{t} w+\partial_{x}(\tilde{u} w)+\partial_{y}(\tilde{v} w)-2 \partial_{y}(\eta w)+\partial_{y}\left(\zeta \int_{0}^{y} w(t, x, \tilde{y}) d \tilde{y}\right)-\partial_{y}^{2} w=0 \\
\left.\left(\partial_{y} w+2 \eta w\right)\right|_{y=0}=0 \\
\left.w\right|_{t=0}=w_{0}(x, y)=\left(\frac{u_{0}}{\partial_{y} \tilde{u}}\right)_{y}(x, y)
\end{array}\right.
$$

where

$$
\eta=\frac{\partial_{y}^{2} \tilde{u}}{\partial_{y} \tilde{u}}, \quad \zeta=\frac{\left(\partial_{t}+\tilde{u} \partial_{x}+\tilde{v} \partial_{y}-\partial_{y}^{2}\right) \partial_{y} \tilde{u}}{\partial_{y} \tilde{u}} .
$$

Similar to the proof for (3.4) in the problem (6.3), it follows that

$$
\|w\|_{\mathcal{A}_{\ell}^{k}\left([0, T] \times \mathbb{R}_{+}^{2}\right)} \leq C(T)\left\|w_{0}\right\|_{\mathcal{A}_{\ell}^{k}\left(\mathbb{R}_{+}^{2}\right)}, \quad k \leq \tilde{k}-3,
$$

for a constant $C(T)$ depending on $T>0$ and the norms of the initial data $u_{0}^{1}, u_{0}^{2}$ in the spaces given in the existence part of Theorem 1.1. 
From (6.4), and the transformation (6.2), we deduce

$$
\left\|u^{1}-u^{2}\right\|_{\mathcal{A}_{\ell}^{k}\left([0, T] \times \mathbb{R}_{+}^{2}\right)}+\left\|v^{1}-v^{2}\right\|_{\mathcal{D}_{0}^{k-1}\left([0, T] \times \mathbb{R}_{+}^{2}\right)} \leq C\left\|\frac{\partial}{\partial y}\left(\frac{u_{0}^{1}-u_{0}^{2}}{\partial_{y} u_{0}^{s}}\right)\right\|_{\mathcal{A}_{\ell}^{k}\left(\mathbb{R}_{+}^{2}\right)},
$$

for all $k \leq \tilde{k}-3$. And this concludes the uniqueness and stability results stated in Theorem 1.1.

\section{Proof of some technical estimates}

Finally, in this section, we give the proofs for Lemmas 5.7, 5.10, 5.11, 5.13, and 5.14 stated in Section 5 about the iteration scheme (4.9) and (4.10).

We start with the proof for Lemma 5.7

Proof of Lemma 5.7; Let us first prove the estimate (5.13). First of all, it holds true for $\delta u^{0}$. Indeed, from

$$
\delta u^{0}=\partial_{y}\left(u^{s}+S_{\theta_{0}} \tilde{u}^{0}\right) \int_{0}^{y} w^{0} d \tilde{y}
$$

by using Lemma 2.1 and the Sobolev embedding theorem, we have

$$
\left\|\delta u^{0}\right\|_{\mathcal{A}_{l}^{k}} \leq C_{k}^{0}\left\|w^{0}\right\|_{\mathcal{A}_{l}^{k}} \leq C_{k}^{0} C_{0} \epsilon \theta_{0}^{\max \{3-\tilde{k}, k-\tilde{k}\}} \Delta \theta_{0}, \quad k \geq 2,
$$

with

$$
C_{k}^{0}=\left\|u^{s}\right\|_{\mathcal{C}_{\ell}^{k+1}}+\left\|\tilde{u}^{0}\right\|_{\mathcal{A}_{\ell}^{k+1}} .
$$

The estimate (5.13) holds obviously for $\delta u^{0}$ when $k=0,1$.

Now, suppose that (5.13) holds for $\delta u^{p}, 0 \leq p \leq j-1$. We estimate $\delta u^{j}$ as follows.

Recalling $w^{j}=\left(\frac{\delta u^{j}}{\partial_{y}\left(u_{\theta_{j}}^{j}\right)}\right)_{y}$, we have

$$
\delta u^{j}=\partial_{y}\left(u_{\theta_{j}}^{j}\right) \int_{0}^{y} w^{j} d \tilde{y} .
$$

Using again Lemma 2.1, the Sobolev embedding theorem and (5.21), it follows that, for $k \geq 4$ and $\tilde{k} \geq 6$,

$$
\begin{aligned}
\left\|\delta u^{j}\right\|_{\mathcal{A}_{l}^{k}} \leq & M_{k}\left(\left(C_{k}^{0}+\left\|\partial_{y}\left(u_{\theta_{j}}^{j}-u^{s}\right)\right\|_{\mathcal{A}_{l}^{k}}\right)\left\|w^{j}\right\|_{\mathcal{A}_{l}^{2}}+\left\|\partial_{y} u_{\theta_{j}}^{j}\right\|_{L_{y, \ell}^{2}\left(L_{t, x}^{\infty}\right)}\left\|w^{j}\right\|_{\mathcal{A}_{l}^{k}}\right) \\
\leq & M_{k}\left(\left(C_{k}^{0}+C_{\rho} \theta_{j} \sum_{p=0}^{j-1}\left\|\delta u^{p}\right\|_{\mathcal{A}_{l}^{k}}\right)\left\|w^{j}\right\|_{\mathcal{A}_{l}^{2}}+\left\|\partial_{y} u^{j}\right\|_{L_{y, \ell}^{2}\left(L_{t, x}^{\infty}\right)}\left\|w^{j}\right\|_{\mathcal{A}_{l}^{k}}\right) \\
\leq & C_{0} M_{k} C_{\rho}\left(C_{k}^{0}+C_{1} \epsilon \theta_{j} \sum_{p=0}^{j-1} \theta_{p}^{\max \{3-\tilde{k}, k-\tilde{k}\}} \Delta \theta_{p}\right) \epsilon \theta_{j}^{3-\tilde{k}} \Delta \theta_{j} \\
& +C_{0} C_{\rho} M_{k}\left(C_{3}^{0}+C_{1} \epsilon \sum_{p=0}^{j-1} \theta_{p}^{3-\tilde{k}} \Delta \theta_{p}\right) \epsilon \theta_{j}^{\max \{3-\tilde{k}, k-\tilde{k}\}} \Delta \theta_{j} \\
\leq & \epsilon C_{0} C_{\rho} M_{k}\left(C_{k}^{0}+C_{1} \tilde{C} \epsilon \theta_{j}^{1+\max \{0, k+1-\tilde{k}\}}\right) \theta_{j}^{3-\tilde{k}} \Delta \theta_{j} \\
& +\epsilon C_{0} C_{\rho} M_{k}\left(C_{3}^{0}+C_{1} \tilde{C} \epsilon\right) \theta_{j}^{\max \{3-\tilde{k}, k-\tilde{k}\}} \Delta \theta_{j} \\
\leq & \epsilon C_{0} C_{\rho} M_{k}\left(C_{k}^{0} \theta_{0}^{-1}+C_{3}^{0}+2 C_{1} \tilde{C} \epsilon\right) \theta_{j}^{k-\tilde{k}} \Delta \theta_{j},
\end{aligned}
$$

where the constant $C_{\rho}$ comes from (4.1). 
By setting $C_{1}=4 C_{0} C_{\rho} M_{k} C_{3}^{0}$, we can choose $0<\epsilon \leq \epsilon_{0}$ and $\theta_{0}>0$ large enough such that

$$
C_{0} C_{\rho} M_{k}\left(C_{k}^{0} \theta_{0}^{-1}+C_{3}^{0}+2 C_{1} \tilde{C} \epsilon\right) \leq C_{1}
$$

Therefore, we get

$$
\left\|\delta u^{j}\right\|_{\mathcal{A}_{l}^{k}} \leq C_{1} \epsilon \theta_{j}^{k-\tilde{k}} \Delta \theta_{j}
$$

for $k \geq 4$. On the other hand, we have

$$
\begin{aligned}
\left\|\delta u^{j}\right\|_{\mathcal{A}_{\ell}^{3}} \leq & M_{k}\left(C_{3}^{0}+C_{\rho} \sum_{p=0}^{j-1}\left\|\delta u^{p}\right\|_{\mathcal{A}_{\ell}^{4}}\right)\left\|w^{j}\right\|_{\mathcal{A}_{\ell}^{2}}+M_{k}\left\|\partial_{y} u^{j}\right\|_{L_{y, \ell}^{2}\left(L_{t, x}^{\infty}\right)}\left\|w^{j}\right\|_{\mathcal{A}_{\ell}^{3}} \\
\leq & C_{0} M_{k} C_{\rho}\left(C_{3}^{0}+C_{1} \epsilon \sum_{p=0}^{j-1} \theta_{p}^{4-\tilde{k}} \Delta \theta_{p}\right) \epsilon \theta_{j}^{3-\tilde{k}} \Delta \theta_{j} \\
& +C_{0} C_{\rho} M_{k}\left(C_{3}^{0}+C_{1} \epsilon \sum_{p=0}^{j-1} \theta_{p}^{3-\tilde{k}} \Delta \theta_{p}\right) \epsilon \theta_{j}^{3-\tilde{k}} \Delta \theta_{j} \\
\leq & C_{1} \epsilon \theta_{j}^{3-\tilde{k}} \Delta \theta_{j},
\end{aligned}
$$

for $\tilde{k} \geq 6$, by choosing a proper constant $C_{1}>0$. And this completes the proof of the estimate (5.13).

We now turn to the estimates (5.14) and (5.15). When $j=0$, from

$$
\frac{\delta u^{0}}{\partial_{y} u^{s}}=\left(1+\frac{\partial_{y} S_{\theta_{0}}\left(\tilde{u}^{0}\right)}{\partial_{y} u^{s}}\right) \int_{0}^{y} w^{0}(t, x, \tilde{y}) d \tilde{y}
$$

we have, by using the Sobolev embedding theorem and for some $\ell>1 / 2$, that

$$
\begin{aligned}
\left\|\frac{\delta u^{0}}{\partial_{y} u^{s}}\right\|_{L^{\infty}} & \leq\left(1+\left\|\frac{\partial_{y} S_{\theta_{0}}\left(\tilde{u}^{0}\right)}{\partial_{y} u^{s}}\right\|_{L^{\infty}}\right) \bar{C}_{\ell}\left\|w^{0}\right\|_{L_{y, \ell}^{2}\left(L_{t, x}^{\infty}\right)} \\
& \leq\left(1+\left\|\frac{\partial_{y} S_{\theta_{0}}\left(\tilde{u}^{0}\right)}{\partial_{y} u^{s}}\right\|_{L^{\infty}}\right) \bar{C}_{\ell}\left\|w^{0}\right\|_{\mathcal{A}_{\ell}^{2}},
\end{aligned}
$$

where $\bar{C}_{\ell}=\left(\int_{0}^{+\infty}\left(1+y^{2}\right)^{-\ell} d y\right)^{\frac{1}{2}}$. The estimate (5.14) with $j=0$ follows immediately by choosing

$$
C_{1} \geq C_{0} \bar{C}_{\ell}\left(1+\left\|\frac{\partial_{y} S_{\theta_{0}}\left(\tilde{u}^{0}\right)}{\partial_{y} u^{s}}\right\|_{L^{\infty}}\right)
$$

Applying Lemma 2.1 to (7.1) gives

$$
\begin{aligned}
& \sum_{k_{1}+\left[\frac{k_{2}+1}{2}\right] \leq k-1}\left\|\partial_{\mathcal{T}}^{k_{1}} \partial_{y}^{k_{2}}\left(\frac{\delta u^{0}}{\partial_{y} u^{s}}\right)\right\|_{L_{y}^{\infty}\left(L_{t, x}^{2}\right)} \\
& \leq M_{k} \bar{C}_{\ell}\left\{\sum_{\substack{1 \leq k_{1}+\left[\frac{k_{2}+1}{2}\right] \leq k-1\\
}}\left\|\partial_{\mathcal{T}}^{k_{1}} \partial_{y}^{k_{2}}\left(\frac{\partial_{y} S_{\theta_{0}}\left(\tilde{u}^{0}\right)}{\partial_{y} u^{s}}\right)\right\|_{L^{\infty}}\left\|w^{0}\right\|_{L_{\ell}^{2}}\right. \\
& \left.+\left(1+\left\|\frac{\partial_{y} S_{\theta_{0}}\left(\tilde{u}^{0}\right)}{\partial_{y} u^{s}}\right\|_{L^{\infty}}\right)\left\|w^{0}\right\|_{\mathcal{A}_{\ell}^{k-1}}\right\} .
\end{aligned}
$$


Then this yields the estimate (5.15) with $j=0$ by choosing

$$
C_{1} \geq M_{k} C_{0} \bar{C}_{\ell}\left\{1+\sum_{0 \leq k_{1}+\left[\frac{k_{2}+1}{2}\right] \leq k-1}\left\|\partial_{\mathcal{T}}^{k_{1}} \partial_{y}^{k_{2}}\left(\left(1+\frac{\partial_{y} S_{\theta_{0}}\left(\tilde{u}^{0}\right)}{\partial_{y} u^{s}}\right)\right)\right\|_{L^{\infty}}\right\}
$$

When $1 \leq j \leq n-1$, by definition, we have

$$
\frac{\delta u^{j}}{\partial_{y} u^{s}}=\frac{\partial_{y} u_{\theta_{j}}^{j}}{\partial_{y} u^{s}} \int_{0}^{y} w^{j}(t, x, \tilde{y}) d \tilde{y}
$$

By using Lemma 2.1 and the assumptions on $w^{j}$, it is sufficient to obtain the bounds of

$$
\frac{\partial_{y} u_{\theta_{j}}^{j}}{\partial_{y} u^{s}}=1+\frac{\partial_{y} S_{\theta_{j}}\left(\tilde{u}^{0}\right)}{\partial_{y} u^{s}}+\sum_{p=0}^{j-1} \frac{\partial_{y} S_{\theta_{j}} \delta u^{p}}{\partial_{y} u^{s}}
$$

in the spaces $L^{\infty}$ and $\mathcal{D}_{0}^{k-1}$, respectively.

To obtain the estimate (5.14) with index $j$, suppose that (5.14) holds for $\frac{\delta u^{p}}{\partial_{y} u^{s}}$ with $0 \leq p \leq j-1$ and prove it by induction. Note that $j=0$ holds by the above argument.

For this, first we show Lemma 7.1.

Lemma 7.1. Suppose that the estimate (5.12) holds for $w^{j}, 0 \leq j \leq n-1$, with $\tilde{k} \geq 7$, then there exists a constant $M_{s}$, such that for all $0 \leq j \leq n$,

$$
\left\|\frac{\partial_{y} u_{\theta_{j}}^{j}}{\partial_{y} u^{s}}\right\|_{L^{\infty}} \leq M_{s}
$$

For continuity of the presentation, we postpone the proof of Lemma 7.1. Then by Lemma 7.1, with (7.2), it follows that

$$
\left\|\frac{\delta u^{j}}{\partial_{y} u^{s}}\right\|_{L^{\infty}} \leq M_{s} \bar{C}_{\ell} C_{0} \epsilon \theta_{j}^{3-\tilde{k}} \Delta \theta_{j} \leq C_{1} \epsilon \theta_{j}^{3-\tilde{k}} \Delta \theta_{j}
$$

with

$$
C_{1} \geq M_{s} \bar{C}_{\ell} C_{0}
$$

To prove (5.15) with index $j$, we also suppose that it holds for $\frac{\delta u^{p}}{\partial_{y} u^{s}}, 0 \leq p \leq j-1$.

Then, obviously, we have the identity

$$
\begin{aligned}
& \sum_{p=0}^{j-1} \frac{\partial_{y} S_{\theta_{j}} \delta u^{p}}{\partial_{y} u^{s}} \\
& \quad=\sum_{p=0}^{j-1}\left\{S_{\theta_{j}}\left(\frac{\partial_{y}\left(\delta u^{p}\right)}{\partial_{y} u^{s}}\right)+\left[\frac{1}{\partial_{y} u^{s}}, S_{\theta_{j}}\right]\left(\partial_{y}\left(\delta u^{p}\right)\right)\right\} \\
& \quad=\sum_{p=0}^{j-1}\left\{S_{\theta_{j}}\left(\partial_{y} \frac{\delta u^{p}}{\partial_{y} u^{s}}\right)+S_{\theta_{j}}\left(\frac{\delta u^{p}}{\partial_{y} u^{s}} \frac{\partial_{y}^{2} u^{s}}{\partial_{y} u^{s}}\right)+\left[\frac{1}{\partial_{y} u^{s}}, S_{\theta_{j}}\right]\left(\partial_{y}\left(\delta u^{p}\right)\right)\right\} .
\end{aligned}
$$


Thus, we have

$$
\begin{aligned}
& \left\|\sum_{p=0}^{j-1} \partial_{\mathcal{T}}^{k_{1}} \partial_{y}^{k_{2}}\left(\frac{\partial_{y} S_{\theta_{j}} \delta u^{p}}{\partial_{y} u^{s}}\right)\right\|_{L_{y}^{\infty}\left(L_{t, x}^{2}\right)} \\
& \leq \tilde{B} \sum_{p=0}^{j-1}\left\{\left\|\partial_{\mathcal{T}}^{k_{1}} \partial_{y}^{k_{2}} S_{\theta_{j}} \partial_{y}\left(\frac{\delta u^{p}}{\partial_{y} u^{s}}\right)\right\|_{L_{y}^{\infty}\left(L_{t, x}^{2}\right)}+\left\|\partial_{\mathcal{T}}^{k_{1}} \partial_{y}^{k_{2}}\left(\frac{\delta u^{p}}{\partial_{y} u^{s}}\right)\right\|_{L_{y}^{\infty}\left(L_{t, x}^{2}\right)}\right\} \\
& \quad \leq \tilde{B} \sum_{p=0}^{j-1}\left\{\theta_{j}\left\|\partial_{\mathcal{T}}^{k_{1}} \partial_{y}^{k_{2}}\left(\frac{\delta u^{p}}{\partial_{y} u^{s}}\right)\right\|_{L_{y}^{\infty}\left(L_{t, x}^{2}\right)}+\left\|\partial_{\mathcal{T}}^{k_{1}} \partial_{y}^{k_{2}}\left(\frac{\delta u^{p}}{\partial_{y} u^{s}}\right)\right\|_{L_{y}^{\infty}\left(L_{t, x}^{2}\right)}\right\},
\end{aligned}
$$

where the constant $\tilde{B}$ depends on the commutators in (7.4) which is independent of $j$ and $p$. Using the induction hypothesis for (5.15), we have

$$
\begin{aligned}
\sum_{p=0}^{j-1} \sum_{k_{1}+\left[\frac{k_{2}+1}{2}\right] \leq k-1}\left\|\partial_{\mathcal{T}}^{k_{1}} \partial_{y}^{k_{2}}\left(\frac{\delta u^{p}}{\partial_{y} u^{s}}\right)\right\|_{L_{y}^{\infty}\left(L_{t, x}^{2}\right)} & \leq C_{1} \epsilon \sum_{p=0}^{j-1} \theta_{p}^{\max \{3-\tilde{k}, k-1-\tilde{k}\}} \Delta \theta_{p} \\
& \leq C_{1} \tilde{C} \epsilon \theta_{j}^{\max \{0, k-\tilde{k}\}} .
\end{aligned}
$$

Therefore, we deduce

$$
\begin{gathered}
\sum_{k_{1}+\left[\frac{k_{2}+1}{2}\right] \leq k-1}\left\|\partial_{\mathcal{T}}^{k_{1}} \partial_{y}^{k_{2}}\left(\frac{\partial_{y} u_{\theta_{j}}^{j}}{\partial_{y} u^{s}}\right)\right\|_{L_{y}^{\infty}\left(L_{t, x}^{2}\right)}\left\{\| \sum_{k_{1}+\left[\frac{k_{2}+1}{2}\right] \leq k-1}\left\{\partial_{\mathcal{T}}^{k_{1}} \partial_{y}^{k_{2}}\left(\frac{\partial_{y} S_{\theta_{j}} \tilde{u}^{0}}{\partial_{y} u^{s}}\right) \|_{L_{y}^{\infty}\left(L_{t, x}^{2}\right)}\right.\right. \\
\left.\quad+\left\|\sum_{p=0}^{j-1} \partial_{\mathcal{T}}^{k_{1}} \partial_{y}^{k_{2}}\left(\frac{\partial_{y} S_{\theta_{j}} \delta u^{p}}{\partial_{y} u^{s}}\right)\right\|_{L_{y}^{\infty}\left(L_{t, x}^{2}\right)}\right\} \\
\leq C^{a} \epsilon+2 \tilde{B} \tilde{C} C_{1} \epsilon \theta_{j}^{\max \{1, k+1-\tilde{k}\}} .
\end{gathered}
$$

Now using Lemma 2.1, it follows that

$$
\begin{aligned}
& \sum_{k_{1}+\left[\frac{k_{2}+1}{2}\right] \leq k-1}\left\|\partial_{\mathcal{T}}^{k_{1}} \partial_{y}^{k_{2}}\left(\frac{\delta u^{j}}{\partial_{y} u^{s}}\right)\right\|_{L_{y}^{\infty}\left(L_{t, x}^{2}\right)} \\
& \leq M_{k}\left\{\sum_{k_{1}+\left[\frac{k_{2}+1}{2}\right] \leq k-1} \bar{C}_{\ell}\left\|\partial_{\mathcal{T}}^{k_{1}} \partial_{y}^{k_{2}}\left(\frac{\partial_{y} u_{\theta_{j}}^{j}}{\partial_{y} u^{s}}\right)\right\|_{L_{y}^{\infty}\left(L_{t, x}^{2}\right)}\left\|w^{j}\right\|_{L_{y, \ell}^{2}\left(L_{t, x}^{\infty}\right)}\right. \\
& \left.+\sum_{k_{1}+\left[\frac{k_{2}+1}{2}\right] \leq k-1}\left\|\left(\frac{\partial_{y} u_{\theta_{j}}^{j}}{\partial_{y} u^{s}}\right)\right\|_{L^{\infty}}\left\|\left(\partial_{\mathcal{T}}^{k_{1}} \partial_{y}^{k_{2}} \int_{0}^{y} w^{j}(t, x, \tilde{y}) d \tilde{y}\right)\right\|_{L_{y}^{\infty}\left(L_{t, x}^{2}\right)}\right\} \\
& \leq M_{k} \bar{C}_{\ell}\left(\left(C^{a}+2 \tilde{B} \tilde{C} C_{1} \theta_{j}^{\max \{1, k+1-\tilde{k}\}}\right) \epsilon\left\|w^{j}\right\|_{\mathcal{A}_{\ell}^{2}}+M_{s}\left\|w^{j}\right\|_{\mathcal{A}_{\ell}^{k-1}}\right) \\
& \leq C_{1} \epsilon \theta_{j}^{\max \{4-\tilde{k}, k-1-\tilde{k}\}} \Delta \theta_{j} \text {, }
\end{aligned}
$$


by choosing $C_{1}$ satisfying

$$
C_{1} \geq M_{k} C_{0} \bar{C}_{\ell}\left(\left(C^{a}+2 \tilde{B} \tilde{C} C_{1}\right) \epsilon+M_{s}\right) .
$$

Then, we have proved (5.15) for $4 \leq k-1 \leq k_{0}-1$.

On the other hand, similar to the argument to derive (7.5) from (7.4), one can deduce

$$
\sum_{k_{1}+\left[\frac{k_{2}+1}{2}\right] \leq 3}\left\|\partial_{\mathcal{T}}^{k_{1}} \partial_{y}^{k_{2}}\left(\frac{\partial_{y} u_{\theta_{j}}^{j}}{\partial_{y} u^{s}}\right)\right\|_{L_{y}^{\infty}\left(L_{t, x}^{2}\right)} \leq C \epsilon,
$$

for a positive constant $C>0$. Thus, as in (7.6), we obtain

$$
\left\|\frac{\delta u^{j}}{\partial_{y} u^{s}}\right\|_{\mathcal{D}_{0}^{3}} \leq C_{1} \epsilon \theta_{j}^{3-\tilde{k}} \Delta \theta_{j} .
$$

This completes the proof of Lemma 5.7

We now turn to the proof of Lemma 7.1 .

Proof of Lemma 7.1: The case when $0 \leq j \leq 1$ is obvious. From (7.4), we have

$$
\begin{aligned}
\left\|\sum_{p=0}^{j-1} \frac{\partial_{y} S_{\theta_{j}} \delta u^{p}}{\partial_{y} u^{s}}\right\|_{L^{\infty}} & \leq \sum_{p=0}^{j-1}\left\{\left\|S_{\theta_{j}}\left(\partial_{y} \frac{\delta u^{p}}{\partial_{y} u^{s}}\right)\right\|_{L^{\infty}}\right. \\
& \left.+\left\|S_{\theta_{j}}\left(\delta u^{p} \frac{\partial_{y}^{2} u^{s}}{\left(\partial_{y} u^{s}\right)^{2}}\right)\right\|_{L^{\infty}}+\left\|\left[\frac{1}{\partial_{y} u^{s}}, S_{\theta_{j}}\right]\left(\partial_{y}\left(\delta u^{p}\right)\right)\right\|_{L^{\infty}}\right\} \\
& \leq \sum_{p=0}^{j-1}\left\{\left\|S_{\theta_{j}}\left(\partial_{y} \frac{\delta u^{p}}{\partial_{y} u^{s}}\right)\right\|_{L^{\infty}}+B\left\|\frac{\delta u^{p}}{\partial_{y} u^{s}}\right\|_{L^{\infty}}\right\},
\end{aligned}
$$

where we have used the estimate of commutators associated with the mollifier $S_{\theta_{j}}$ given in Lemma 4.1

Suppose now (7.3) holds for $0 \leq p \leq j-1$. Let us check the case when $p=j$. Obviously, we have

$$
\begin{aligned}
S_{\theta_{j}}\left(\partial_{y} \frac{\delta u^{p}}{\partial_{y} u^{s}}\right) & =S_{\theta_{j}} \partial_{y}\left(\frac{\partial_{y} u_{\theta_{p}}^{p}}{\partial_{y} u^{s}} \int_{0}^{y} w^{p}(t, x, \tilde{y}) d \tilde{y}\right) \\
& =S_{\theta_{j}}\left(\left(\partial_{y} \frac{\partial_{y} u_{\theta_{p}}^{p}}{\partial_{y} u^{s}}\right) \int_{0}^{y} w^{p}(t, x, \tilde{y}) d \tilde{y}\right)+S_{\theta_{j}}\left(\frac{\partial_{y} u_{\theta_{p}}^{p}}{\partial_{y} u^{s}} w^{p}\right) .
\end{aligned}
$$

For the second term given on the right hand side of (7.7), using the induction hypothesis, we get

$$
\begin{aligned}
\sum_{p=0}^{j-1}\left\|S_{\theta_{j}}\left(\frac{\partial_{y} u_{\theta_{p}}^{p}}{\partial_{y} u^{s}} w^{p}\right)\right\|_{L^{\infty}} & \leq \sum_{p=0}^{j-1}\left\|\frac{\partial_{y} u_{\theta_{p}}^{p}}{\partial_{y} u^{s}}\right\|_{L^{\infty}}\left\|w^{p}\right\|_{L^{\infty}} \\
& \leq M_{s} \sum_{p=0}^{j-1}\left\|w^{p}\right\|_{\mathcal{A}_{0}^{2}} \leq M_{s} C_{0} \epsilon \sum_{p=0}^{j-1} \theta_{p}^{3-\tilde{k}} \Delta \theta_{p} \leq M_{s} C_{0} \tilde{C} \epsilon .
\end{aligned}
$$


For the first term on the right hand side of (7.7), using Lemma 4.1 when $\tilde{k} \geq 7$ gives

$$
\begin{aligned}
& \sum_{p=0}^{j-1}\left\|S_{\theta_{j}}\left(\left(\partial_{y} \frac{\partial_{y} u_{\theta_{p}}^{p}}{\partial_{y} u^{s}}\right) \int_{0}^{y} w^{p}(t, x, \tilde{y}) d \tilde{y}\right)\right\|_{L^{\infty}} \\
& =\sum_{p=0}^{j-1}\left\|S_{\theta_{j}}\left\{\partial_{y}\left(\frac{\partial_{y} S_{\theta_{p}} \tilde{u}^{0}}{\partial_{y} u^{s}}+\sum_{q=0}^{p-1} \frac{\partial_{y} S_{\theta_{p}} \delta u^{q}}{\partial_{y} u^{s}}\right) \int_{0}^{y} w^{p}(t, x, \tilde{y}) d \tilde{y}\right\}\right\|_{L^{\infty}} \\
& \leq \sum_{p=0}^{j-1}\left\{\overline { C } _ { \ell } \| w ^ { p } \| _ { L _ { y , \ell } ^ { 2 } ( L _ { t , x } ^ { \infty } ) } \left(\left\|\partial_{y} \frac{\partial_{y} S_{\theta_{p}} \tilde{u}^{0}}{\partial_{y} u^{s}}\right\|_{L^{\infty}}+\sum_{q=0}^{p-1}\left(\theta_{p}^{2}\left\|\frac{\delta u^{q}}{\partial_{y} u^{s}}\right\|_{L^{\infty}}\right.\right.\right. \\
& \left.\left.\left.\quad+\left\|\partial_{y}\left[\frac{1}{\partial_{y} u^{s}}, \partial_{y} S_{\theta_{p}}\right] \delta u^{q}\right\|_{L^{\infty}}\right)\right)\right\} \\
& \leq \tilde{C} \bar{C}_{\ell} C_{0} \epsilon \sum_{p=0}^{j-1} \theta_{p}^{3-\tilde{k}} \Delta \theta_{p} \leq \tilde{C}^{2} \bar{C}_{\ell} C_{0} \epsilon .
\end{aligned}
$$

In summary, we conclude

$$
\begin{aligned}
\left\|\frac{\partial_{y} u_{\theta_{j}}^{j}}{\partial_{y} u^{s}}\right\|_{L^{\infty}} & \leq 1+\left\|\frac{\partial_{y} \tilde{u}_{\theta_{j}}^{0}}{\partial_{y} u^{s}}\right\|_{L^{\infty}}+\left\|\sum_{p=0}^{j-1} \frac{\partial_{y} S_{\theta_{j}} \delta u^{p}}{\partial_{y} u^{s}}\right\|_{L^{\infty}} \\
& \leq 1+C^{a} \epsilon+\tilde{C}^{2} \bar{C}_{\ell} C_{0} \epsilon+M_{s} C_{0} \tilde{C} \epsilon+B C_{1} \tilde{C} \epsilon
\end{aligned}
$$

which implies the estimate (7.3) by choosing

$$
M_{s} \geq 2\left(1+C^{a} \epsilon+\tilde{C}^{2} \bar{C}_{\ell} C_{0} \epsilon+B C_{1} \tilde{C} \epsilon\right)
$$

and

$$
0<\epsilon \leq \frac{1}{2 C_{0} \tilde{C}}
$$

To prove Lemma 5.10 for $\eta^{n}$, we need the following lemma.

Lemma 7.2. Under the assumptions of Lemma 7.1, there exists a constant $C_{11}$, such that for all $0 \leq j \leq n$,

$$
\left\|\frac{\partial_{y}\left(u_{\theta_{j}}^{j}-u^{s}\right)}{\partial_{y} u^{s}}\right\|_{\mathcal{A}_{\ell}^{k}} \leq C_{11} \epsilon \theta_{j}^{\max \{0, k+1-\tilde{k}\}} .
$$

Proof: This lemma can also be proved by induction on $j$. Suppose that it holds for $0 \leq p \leq j-1$, and let us study the case when $p=j$.

From the identity

$$
\frac{\partial_{y}\left(u_{\theta_{j}}^{j}-u^{s}\right)}{\partial_{y} u^{s}}=\sum_{p=0}^{j-1} \frac{\partial_{y} S_{\theta_{j}} \delta u^{p}}{\partial_{y} u^{s}}+\frac{\partial_{y} S_{\theta_{j}} \tilde{u}^{0}}{\partial_{y} u^{s}},
$$


we have

$$
\begin{aligned}
\left\|\frac{\partial_{y}\left(u_{\theta_{j}}^{j}-u^{s}\right)}{\partial_{y} u^{s}}\right\|_{\mathcal{A}_{\ell}^{k}} \leq & \left\|\sum_{p=0}^{j-1} \frac{\partial_{y} S_{\theta_{j}} \delta u^{p}}{\partial_{y} u^{s}}\right\|_{\mathcal{A}_{\ell}^{k}}+\left\|\frac{\partial_{y} S_{\theta_{j}} \tilde{u}^{0}}{\partial_{y} u^{s}}\right\|_{\mathcal{A}_{\ell}^{k}} \\
\leq & \sum_{p=0}^{j-1}\left\{\left\|S_{\theta_{j}}\left(\partial_{y} \frac{\delta u^{p}}{\partial_{y} u^{s}}\right)\right\|_{\mathcal{A}_{\ell}^{k}}+\left\|S_{\theta_{j}}\left(\delta u^{p} \frac{\partial_{y}^{2} u^{s}}{\left(\partial_{y} u^{s}\right)^{2}}\right)\right\|_{\mathcal{A}_{\ell}^{k}}\right. \\
& \left.+\left\|\left[\frac{1}{\partial_{y} u^{s}}, S_{\theta_{j}}\right]\left(\partial_{y}\left(\delta u^{p}\right)\right)\right\|_{\mathcal{A}_{\ell}^{k}}\right\}+\left\|\frac{\partial_{y} S_{\theta_{j}} \tilde{u}^{0}}{\partial_{y} u^{s}}\right\|_{\mathcal{A}_{\ell}^{k}} \\
& \leq \sum_{p=0}^{j-1}\left\{\left\|S_{\theta_{j}}\left(\partial_{y} \frac{\delta u^{p}}{\partial_{y} u^{s}}\right)\right\|_{\mathcal{A}_{\ell}^{k}}+\tilde{B}\left\|\frac{\delta u^{p}}{\partial_{y} u^{s}}\right\|_{\mathcal{A}_{\ell}^{k}}\right\}+\left\|\frac{\partial_{y} S_{\theta_{j}} \tilde{u}^{0}}{\partial_{y} u^{s}}\right\|_{\mathcal{A}_{\ell}^{k}}
\end{aligned}
$$

To estimate $\left\|S_{\theta_{j}}\left(\partial_{y} \frac{\delta u^{p}}{\partial_{y} u^{s}}\right)\right\|_{\mathcal{A}_{\ell}^{k}}$, we use the relation (7.7). For the second term on the right hand side of (7.7), by using (7.3) we have

$$
\begin{aligned}
& \sum_{p=0}^{j-1}\left\|S_{\theta_{j}}\left(\frac{\partial_{y} u_{\theta_{p}}^{p}}{\partial_{y} u^{s}} w^{p}\right)\right\|_{\mathcal{A}_{\ell}^{k}} \\
& \quad \leq M_{k} \sum_{p=0}^{j-1}\left\{\left\|\frac{\partial_{y} u_{\theta_{p}}^{p}}{\partial_{y} u^{s}}\right\|_{L^{\infty}}\left\|w^{p}\right\|_{\mathcal{A}_{\ell}^{k}}+\left\|\frac{\partial_{y}\left(u_{\theta_{p}}^{p}-u^{s}\right)}{\partial_{y} u^{s}}\right\|_{\mathcal{A}_{\ell}^{k}}\left\|w^{p}\right\|_{L^{\infty}}\right\} \\
& \quad \leq M_{k} \sum_{p=0}^{j-1}\left\{M_{s} C_{0} \epsilon \theta_{p}^{\max \{3-\tilde{k}, k-\tilde{k}\}} \Delta \theta_{p}+C_{11} C_{0} \epsilon^{2} \theta_{p}^{\max \{3-\tilde{k}, k+4-2 \tilde{k}\}} \Delta \theta_{p}\right\} \\
& \quad \leq M_{k} \tilde{C} C_{0} \epsilon\left(M_{s}+C_{11} \epsilon\right) \theta_{j}^{\max \{0, k+1-\tilde{k}\}} .
\end{aligned}
$$

For the first term on the right hand side of (7.7), we have for $k \geq 4$,

$$
\begin{aligned}
& \sum_{p=0}^{j-1}\left\|S_{\theta_{j}}\left(\left(\partial_{y} \frac{\partial_{y} u_{\theta_{p}}^{p}}{\partial_{y} u^{s}}\right) \int_{0}^{y} w^{p}(t, x, \tilde{y}) d \tilde{y}\right)\right\|_{\mathcal{A}_{\ell}^{k}} \\
& \leq M_{k} \theta_{j} \bar{C}_{\ell} \sum_{p=0}^{j-1}\left\{\left\|w^{p}\right\|_{L_{y, \ell}^{2}\left(L_{t, x}^{\infty}\right)}\left\|\partial_{y} \frac{\partial_{y}\left(u_{\theta_{p}}^{p}-u^{s}\right)}{\partial_{y} u^{s}}\right\|_{\mathcal{A}_{\ell}^{k-1}}\right. \\
& \left.+\left\|w^{p}\right\|_{\mathcal{A}_{\ell}^{k-1}}\left\|\partial_{y} \frac{\partial_{y} u_{\theta_{p}}^{p}}{\partial_{y} u^{s}}\right\|_{L_{y, \ell}^{2}\left(L_{t, x}^{\infty}\right)}\right\} \\
& \leq M_{k} \theta_{j} \bar{C}_{\ell} \sum_{p=0}^{j-1}\left\{\left\|w^{p}\right\|_{\mathcal{A}_{\ell}^{2}}\left\|\frac{\partial_{y}\left(u_{\theta_{p}}^{p}-u^{s}\right)}{\partial_{y} u^{s}}\right\|_{\mathcal{A}_{\ell}^{k}}+\left\|w^{p}\right\|_{\mathcal{A}_{\ell}^{k}}\left\|\frac{\partial_{y}\left(u_{\theta_{p}}^{p}-u^{s}\right)}{\partial_{y} u^{s}}\right\|_{\mathcal{A}_{\ell}^{3}}\right\} \\
& \leq M_{k} \theta_{j} \bar{C}_{\ell} \sum_{p=0}^{j-1}\left\{C_{0} \theta_{p}^{3-\tilde{k}} \Delta \theta_{p} C_{11} \epsilon^{2} \theta_{p}^{\max \{0, k+1-\tilde{k}\}}+C_{0} \theta_{p}^{\max \{3-\tilde{k}, k-\tilde{k}\}} \Delta \theta_{p} C_{11} \epsilon^{2}\right\} \\
& \leq 2 M_{k} \bar{C}_{\ell} C_{0} \tilde{C} C_{11} \epsilon^{2} \theta_{j}^{\max \{1, k+2-\tilde{k}\}},
\end{aligned}
$$


and

$$
\sum_{p=0}^{j-1}\left\|S_{\theta_{j}}\left(\left(\partial_{y} \frac{\partial_{y} u_{\theta_{p}}^{p}}{\partial_{y} u^{s}}\right) \int_{0}^{y} w^{p}(t, x, \tilde{y}) d \tilde{y}\right)\right\|_{\mathcal{A}_{\ell}^{3}} \leq B_{1} \epsilon^{2},
$$

for a positive constant $B_{1}$.

By plugging the above three estimates into the first term on the right hand side of (7.10), (7.9) follows by choosing a proper constant $C_{11}>0$.

Remark 7.3. From the above argument, it is easy to see that the estimates (7.3) and (7.9) hold without mollifying $(\cdot)_{\theta_{j}}$, but for $k \leq k_{0}-1$.

We are now ready to prove Lemma 5.13 .

Proof of Lemma 5.13: From the estimate (7.8), we get immediately that there is $\epsilon_{0}>0$ such that when $0<\epsilon \leq \epsilon_{0}$, it holds that

$$
\left\|\frac{\partial_{y} u_{\theta_{j}}^{j}}{\partial_{y} u^{s}}\right\|_{L^{\infty}} \leq 2, \quad \inf \left|\frac{\partial_{y} u_{\theta_{j}}^{j}}{\partial_{y} u^{s}}\right| \geq \frac{1}{2},
$$

for $0 \leq j \leq n$. Hence, the first estimate given in (5.27) follows.

By using the following Fà Di Bruno formula,

$$
\partial^{m}(g(f))=m ! \sum_{1 \leq r \leq m} \frac{1}{r !} g^{(r)}(f) \prod_{m_{1}+\cdots m_{r}=m, m_{j} \geq 1} \frac{1}{m_{j} !} \partial^{m_{j}} f
$$

we have

$$
\begin{aligned}
\left\|\left(\frac{\partial_{y} u_{\theta_{n}}^{n}}{\partial_{y} u^{s}}\right)^{-1}\right\| & \leq B_{k} \sum_{1 \leq k_{1}+\left[\frac{k_{2}+1}{2}\right] \leq k} \sum_{1 \leq r \leq k_{1}+k_{2}}\left\|\prod_{1 \leq j \leq r} \partial_{\mathcal{T}}^{m_{j}^{1}} \partial_{y}^{m_{j}^{2}}\left(\frac{\partial_{y} u_{\theta_{n}}^{n}}{\partial_{y} u^{s}}\right)\right\|_{L^{2}} \\
\leq & B_{k} \sum_{1 \leq k_{1}+\left[\frac{k_{2}+1}{2}\right] \leq k}\left\|\sum_{1 \leq r \leq k_{1}+k_{2}}\right\| \partial_{\mathcal{T}}^{m_{r}^{1}} \partial_{y}^{m_{r}^{2}}\left(\frac{\partial_{y}\left(u_{\theta_{n}}^{n}-u^{s}\right)}{\partial_{y} u^{s}}\right) \|_{L^{2}} \\
& \times \prod_{1 \leq j \leq r-1}\left\|\partial_{\mathcal{T}}^{m_{j}^{1}} \partial_{y}^{m_{j}^{2}}\left(\frac{\partial_{y}\left(u_{\theta_{n}}^{n}-u^{s}\right)}{\partial_{y} u^{s}}\right)\right\|_{L^{\infty}}\left\|\left(\frac{\partial_{y}\left(u_{\theta_{n}}^{n}-u^{s}\right)}{\partial_{y} u^{s}}\right)\right\|_{\left.\mathcal{A}_{0}^{m_{r}^{1}+\left[\frac{m_{r}^{2}+1}{2}\right.}\right]} \\
\leq & B_{k} \sum_{1 \leq k_{1}+\left[\frac{k_{2}+1}{2}\right] \leq k} \sum_{1 \leq r \leq k_{1}+k_{2}}\left\|\left(\frac{\partial_{y}\left(u_{\theta_{n}}^{n}-u^{s}\right)}{\partial_{y} u^{s}}\right)\right\| \|_{\mathcal{A}_{0}^{m_{j}^{1}+2+\left[\frac{m_{j}^{2}+2}{2}\right]}} \\
& \times \prod_{1 \leq j \leq r-1} \|\left(\frac{1}{}\right.
\end{aligned}
$$

where $m_{1}^{1}+\cdots m_{r}^{1}=k_{1}, m_{1}^{2}+\cdots m_{r}^{2}=k_{2}, m_{j}^{1}+m_{j}^{2} \geq 1$, with $m_{r}^{1}$ and $m_{r}^{2}$ being supposed the largest integers in the corresponding group of indices, respectively. Then, by using (7.9) in the above inequality, it follows that

$$
\left\|\left(\frac{\partial_{y} u_{\theta_{n}}^{n}}{\partial_{y} u^{s}}\right)^{-1}\right\|_{\dot{\mathcal{A}}_{0}^{k}} \leq C_{7} \epsilon \theta_{n}^{\max \{0, k+1-\tilde{k}\}},
$$

for a positive constant $C_{7}>0$. This completes the proof of the lemma.

Now, we turn to the estimates on $\eta^{n}=\frac{\partial_{y}^{2} u_{\theta_{n}}^{n}}{\partial_{y} u_{\theta_{n}}^{n}}$ stated in Lemma 5.10. 
Proof of Lemma 5.10: Obviously, we have

$$
\begin{aligned}
\left\|\eta^{n}-\bar{\eta}^{n}\right\|_{\mathcal{A}_{\ell}^{k}}= & \left\|\frac{\partial_{y}^{2}\left(u_{\theta_{n}}^{n}-u^{s}\right)}{\partial_{y} u_{\theta_{n}}^{n}}\right\|_{\mathcal{A}_{\ell}^{k}} \\
\leq & M_{k}\left(\left\|\frac{\partial_{y}^{2}\left(u_{\theta_{n}}^{n}-u^{s}\right)}{\partial_{y} u^{s}}\right\|_{\mathcal{A}_{\ell}^{k}}\left\|\left(\frac{\partial_{y} u_{\theta_{n}}^{n}}{\partial_{y} u^{s}}\right)^{-1}\right\|_{L^{\infty}}\right. \\
& \left.+\left\|\frac{\partial_{y}^{2}\left(u_{\theta_{n}}^{n}-u^{s}\right)}{\partial_{y} u^{s}}\right\|_{L_{\ell}^{\infty}}\left\|\frac{\partial_{y} u^{s}}{\partial_{y} u_{\theta_{n}}^{n}}\right\|_{\dot{\mathcal{A}}_{0}^{k}}\right) .
\end{aligned}
$$

We now estimate term by term on the right hand side of (7.13). Since

$$
\frac{\partial_{y}^{2}\left(u_{\theta_{n}}^{n}-u^{s}\right)}{\partial_{y} u^{s}}=\partial_{y}\left(\frac{\partial_{y}\left(u_{\theta_{n}}^{n}-u^{s}\right)}{\partial_{y} u^{s}}\right)+\frac{\partial_{y}\left(u_{\theta_{n}}^{n}-u^{s}\right)}{\partial_{y} u^{s}} \frac{\partial_{y}^{2} u^{s}}{\partial_{y} u^{s}},
$$

by using (7.9), we have for $k \geq 4$

$$
\begin{aligned}
\left\|\frac{\partial_{y}^{2}\left(u_{\theta_{n}}^{n}-u^{s}\right)}{\partial_{y} u^{s}}\right\|_{\mathcal{A}_{\ell}^{k}} & \leq M_{k}\left\|\frac{\partial_{y}^{2} u^{s}}{\partial_{y} u^{s}}\right\|_{\mathcal{C}_{0}^{k}}\left\|\frac{\partial_{y}\left(u_{\theta_{n}}^{n}-u^{s}\right)}{\partial_{y} u^{s}}\right\|_{\mathcal{A}_{\ell}^{k}}+\left\|\partial_{y}\left(\frac{\partial_{y}\left(u_{\theta_{n}}^{n}-u^{s}\right)}{\partial_{y} u^{s}}\right)\right\|_{\mathcal{A}_{\ell}^{k}} \\
& \leq M_{k}\left\|\frac{\partial_{y}^{2} u^{s}}{\partial_{y} u^{s}}\right\|_{\mathcal{C}_{0}^{k}}\left\|\frac{\partial_{y}\left(u_{\theta_{n}}^{n}-u^{s}\right)}{\partial_{y} u^{s}}\right\|_{\mathcal{A}_{\ell}^{k}}+C_{\rho} \theta_{n}\left\|\left(\frac{\partial_{y}\left(u^{n}-u^{s}\right)}{\partial_{y} u^{s}}\right)\right\|_{\mathcal{A}_{\ell}^{k}} \\
& \leq \tilde{C}_{11} \epsilon \theta_{n}^{1+\max \{0, k+1-\tilde{k}\}} .
\end{aligned}
$$

On the other hand, by using (7.9), it holds that

$$
\begin{aligned}
\left\|\frac{\partial_{y}^{2}\left(u_{\theta_{n}}^{n}-u^{s}\right)}{\partial_{y} u^{s}}\right\|_{\mathcal{A}_{\ell}^{3}} & \leq M_{3}\left\|\frac{\partial_{y}^{2} u^{s}}{\partial_{y} u^{s}}\right\|_{\mathcal{C}_{0}^{3}}\left\|\frac{\partial_{y}\left(u_{\theta_{n}}^{n}-u^{s}\right)}{\partial_{y} u^{s}}\right\|_{\mathcal{A}_{\ell}^{3}}+\left\|\frac{\partial_{y}\left(u_{\theta_{n}}^{n}-u^{s}\right)}{\partial_{y} u^{s}}\right\|_{\mathcal{A}_{\ell}^{4}} \\
& \leq\left(M_{3}\left\|\frac{\partial_{y}^{2} u^{s}}{\partial_{y} u^{s}}\right\|_{\mathcal{C}_{0}^{3}}+1\right) C_{11} \epsilon .
\end{aligned}
$$

Plugging the estimates (7.14), (7.15), and (7.12) into (7.13), we obtain the estimate (5.24) given in Lemma 5.10 .

To derive the estimate (5.25), from the definition of $\bar{\eta}^{n}$, we have

$$
\begin{aligned}
\left\|\bar{\eta}^{n}\right\|_{\mathcal{C}_{\ell}^{k}} & \leq M_{k}\left(\left\|\frac{\partial_{y}^{2} u^{s}}{\partial_{y} u^{s}}\right\|_{\mathcal{C}_{\ell}^{k}}\left\|\left(\frac{\partial_{y} u_{\theta_{n}}^{n}}{\partial_{y} u^{s}}\right)^{-1}\right\|_{L^{\infty}}+\left\|\frac{\partial_{y}^{2} u^{s}}{\partial_{y} u^{s}}\right\|_{L_{\ell}^{\infty}}\left\|\left(\frac{\partial_{y} u_{\theta_{n}}^{n}}{\partial_{y} u^{s}}\right)^{-1}\right\|_{\dot{\mathcal{C}}_{0}^{k}}\right) \\
& \leq M_{k}\left(\left\|\frac{\partial_{y}^{2} u^{s}}{\partial_{y} u^{s}}\right\|_{\mathcal{C}_{\ell}^{k}}\left\|\left(\frac{\partial_{y} u_{\theta_{n}}^{n}}{\partial_{y} u^{s}}\right)^{-1}\right\|_{L^{\infty}}+\left\|\frac{\partial_{y}^{2} u^{s}}{\partial_{y} u^{s}}\right\|_{L_{\ell}^{\infty}}\left\|\left(\frac{\partial_{y} u_{\theta_{n}}^{n}}{\partial_{y} u^{s}}\right)^{-1}\right\|_{\mathcal{A}_{0}^{k+2}}\right) \\
& \leq M_{k}\left(2\left\|\frac{\partial_{y}^{2} u^{s}}{\partial_{y} u^{s}}\right\|_{\mathcal{C}_{\ell}^{k}}+\left\|\frac{\partial_{y}^{2} u^{s}}{\partial_{y} u^{s}}\right\|_{L_{\ell}^{\infty}} C_{7} \epsilon \theta_{n}^{\max \{0, k+3-\tilde{k}\}}\right),
\end{aligned}
$$

where we have used (7.12) and (7.11). Thus, we get the estimate (5.25) immediately. And this completes the proof of the lemma. 
To estimate $\zeta^{n}$, similar to the proof for Lemma 7.1 from

$$
\begin{aligned}
\frac{\partial_{x y}^{2} S_{\theta_{j}} u^{j}}{\partial_{y} u^{s}}= & \frac{\partial_{x y}^{2}\left(S_{\theta_{j}} \tilde{u}^{0}\right)}{\partial_{y} u^{s}}+\sum_{p=0}^{j-1}\left(S_{\theta_{j}}\left(\frac{\partial_{x y}^{2} \delta u^{p}}{\partial_{y} u^{s}}\right)+\left[\frac{1}{\partial_{y} u^{s}}, S_{\theta_{j}}\right] \partial_{x y}^{2} \delta u^{p}\right) \\
= & \frac{\partial_{x y}^{2}\left(S_{\theta_{j}} \tilde{u}^{0}\right)}{\partial_{y} u^{s}} \\
& +\sum_{p=0}^{j-1}\left(S_{\theta_{j}}\left(\partial_{x} w^{p}+\partial_{x}\left(\frac{\delta u^{p}}{\partial_{y} u^{s}}\right) \frac{\partial_{y}^{2} u^{s}}{\partial_{y} u^{s}}\right)+\left[\frac{1}{\partial_{y} u^{s}}, S_{\theta_{j}}\right] \partial_{x y}^{2} \delta u^{p}\right),
\end{aligned}
$$

we have Lemma 7.4.

Lemma 7.4. Under the assumptions of Lemma 7.1, there is a constant $C_{12}>0$, such that

$$
\left\|\frac{\partial_{x y}^{2} u_{\theta_{j}}^{j}}{\partial_{y} u_{\theta_{j}}^{j}}\right\|_{\mathcal{A}_{\ell}^{k}} \leq C_{12} \epsilon \theta_{j}^{1+\max \{0, k+1-\tilde{k}\}}
$$

and

$$
\left\|\frac{\partial_{x y}^{2} u_{\theta_{j}}^{j}}{\partial_{y} u_{\theta_{j}}^{j}}\right\|_{\mathcal{A}_{\ell}^{3}} \leq C_{12} \epsilon
$$

hold for all $0 \leq j \leq n$.

With this, we are now ready to prove Lemma 5.11 .

Proof of Lemma 5.11; First of all, note that

$$
\begin{aligned}
\left\|\frac{v_{\theta_{n}}^{n} \partial_{y}^{2} u_{\theta_{n}}^{n}}{\partial_{y} u_{\theta_{n}}^{n}}\right\|_{\mathcal{A}_{\ell}^{k}} \leq & M_{k}\left(\left\|v_{\theta_{n}}^{n}\right\|_{L^{\infty}}\left\|\eta^{n}-\bar{\eta}^{n}\right\|_{\mathcal{A}_{\ell}^{k}}+\left\|v_{\theta_{n}}^{n}\right\|_{L_{y}^{\infty}\left(L_{t, x}^{2}\right)}\left\|\bar{\eta}^{n}\right\|_{\mathcal{C}_{\ell}^{k}}\right. \\
& \left.+\left\|\eta^{n}\right\|_{L_{y, \ell}^{2}\left(L_{t, x}^{\infty}\right)}\left\|v_{\theta_{n}}^{n}\right\|_{\mathcal{D}_{0}^{k}}\right) .
\end{aligned}
$$

By using Lemma 5.9 and Lemma 5.10 , we get

$$
\begin{aligned}
\left\|\frac{v_{\theta_{n}}^{n} \partial_{y}^{2} u_{\theta_{n}}^{n}}{\partial_{y} u_{\theta_{n}}^{n}}\right\|_{\mathcal{A}_{\ell}^{k}} \leq & M_{k}\left(C_{3} C_{4} \epsilon \theta_{n}^{\max \{1, k+2-\tilde{k}\}}+C_{3} C_{4}\left(1+\epsilon \theta_{n}^{\max \{0, k+3-\tilde{k}\}}\right)\right. \\
& \left.+2 C_{4} C_{3} \epsilon \theta_{n}^{\max \{1, k+2-\tilde{k}\}}\right) \\
\leq & C_{5} \theta_{n}^{\max \{1, k+3-\tilde{k}\}},
\end{aligned}
$$

when $k \geq 4$ for a positive constant $C_{5}$. Moreover,

$$
\left\|\frac{v_{\theta_{n}}^{n} \partial_{y}^{2} u_{\theta_{n}}^{n}}{\partial_{y} u_{\theta_{n}}^{n}}\right\|_{\mathcal{A}_{\ell}^{3}} \leq M_{3}\left(C_{3} C_{4} \epsilon+C_{3} C_{4}(1+\epsilon)+2 C_{3} C_{4} \epsilon\right) \leq C_{8}
$$


Similarly, by using (2.1) and Lemma 7.4, we can show that

$$
\begin{aligned}
\left\|\frac{u_{\theta_{n}}^{n} \partial_{x y}^{2} u_{\theta_{n}}^{n}}{\partial_{y} u_{\theta_{n}}^{n}}\right\|_{\mathcal{A}_{\ell}^{k}} \leq & M_{k}\left(\left\|u_{\theta_{n}}^{n}\right\|_{L^{\infty}}\left\|\frac{\partial_{x y}^{2} u_{\theta_{n}}^{n}}{\partial_{y} u_{\theta_{n}}^{n}}\right\|_{\mathcal{A}_{\ell}^{k}}+\left\|\frac{\partial_{x y}^{2} u_{\theta_{n}}^{n}}{\partial_{y} u_{\theta_{n}}^{n}}\right\|_{L^{\infty}}\left\|u_{\theta_{n}}^{n}-u^{s}\right\|_{\mathcal{A}_{\ell}^{k}}\right. \\
& \left.+\left\|\frac{\partial_{x y}^{2} u_{\theta_{n}}^{n}}{\partial_{y} u_{\theta_{n}}^{n}}\right\|_{L_{y}^{\infty}\left(L_{t, x}^{2}\right)}\left\|u^{s}\right\|_{\mathcal{C}_{\ell}^{k}}\right) \\
\leq & M_{k}\left(C_{s}\left(C^{a}+C_{3} \epsilon\right) C_{12} \epsilon \theta_{n}^{1+\max \{0, k+1-\tilde{k}\}}\right. \\
& \left.+C_{s}\left(C^{a}+C_{3} \epsilon \theta_{n}^{\max \{0, k+1-\tilde{k}\}}\right) C_{12} \epsilon\right) \\
\leq & C_{5} \theta_{n}^{\max \{1, k+2-\tilde{k}\}}
\end{aligned}
$$

By noticing that

$$
\frac{\left(\partial_{t}-\partial_{y}^{2}\right) \partial_{y} u_{\theta_{n}}^{n}}{\partial_{y} u_{\theta_{n}}^{n}}=\frac{\left(\partial_{t}-\partial_{y}^{2}\right) \partial_{y} u_{\theta_{n}}^{n}}{\partial_{y} u^{s}} \cdot \frac{\partial_{y} u^{s}}{\partial_{y} u_{\theta_{n}}^{n}}=\frac{\left(\partial_{t}-\partial_{y}^{2}\right) \partial_{y}\left(u_{\theta_{n}}^{n}-u^{s}\right)}{\partial_{y} u^{s}} \frac{\partial_{y} u^{s}}{\partial_{y} u_{\theta_{n}}^{n}},
$$

using (7.9) and (7.12), we have

$$
\left\|\frac{\left(\partial_{t}-\partial_{y}^{2}\right) \partial_{y} u_{\theta_{n}}^{n}}{\partial_{y} u_{\theta_{n}}^{n}}\right\|_{\mathcal{A}_{\ell}^{k}} \leq C_{5} \theta_{n}^{\max \{0, k+2-\tilde{k}\}} .
$$

Plugging the above estimates into the definition of $\zeta^{n}$ in (5.23), it leads to the (5.26) given in Lemma 5.11 and then completes its proof.

Finally, let us give the proof of Lemma 5.14,

Proof of Lemma 5.14: Recall the definition

$$
e_{n}^{(1)}=\delta u^{n} \partial_{x} \delta u^{n}+\delta v^{n} \partial_{y} \delta u^{n},
$$

and

$$
\begin{aligned}
e_{n}^{(2)}= & \left(\left(1-S_{\theta_{n}}\right)\left(u^{n}-u^{s}\right)\right) \partial_{x}\left(\delta u^{n}\right)+\delta u^{n} \partial_{x}\left(\left(1-S_{\theta_{n}}\right)\left(u^{n}-u^{s}\right)\right) \\
& +\delta v^{n} \partial_{y}\left(\left(1-S_{\theta_{n}}\right)\left(u^{n}-u^{s}\right)\right)+\left(\left(1-S_{\theta_{n}}\right) v^{n}\right) \partial_{y}\left(\delta u^{n}\right) .
\end{aligned}
$$

We get

$$
\begin{aligned}
\left\|\frac{e_{j}^{(1)}}{\partial_{y} u^{s}}\right\|_{\mathcal{A}_{\ell}^{k_{1}}} \leq & M_{k_{1}}\left\{\left\|\partial_{x} \delta u^{j}\right\|_{\mathcal{A}_{\ell}^{k_{1}}}\left\|\frac{\delta u^{j}}{\partial_{y} u^{s}}\right\|_{L^{\infty}}+\left\|\partial_{x} \delta u^{j}\right\|_{L_{y, \ell}^{2}\left(L_{t, x}^{\infty}\right)}\left\|\frac{\delta u^{j}}{\partial_{y} u^{s}}\right\|_{\mathcal{D}_{\ell}^{k_{1}}}\right. \\
& \left.+\left\|\delta v^{j}\right\|_{L^{\infty}}\left\|\frac{\partial_{y} \delta u^{j}}{\partial_{y} u^{s}}\right\|_{\mathcal{A}_{\ell}^{k_{1}}}+\left\|\frac{\partial_{y} \delta u^{j}}{\partial_{y} u^{s}}\right\|_{L_{y, l}^{2}\left(L_{t, x}^{\infty}\right)}\left\|\delta v^{j}\right\|_{\mathcal{D}_{0}^{k_{1}}}\right\} .
\end{aligned}
$$

Obviously, from

$$
\frac{\partial_{y} \delta u^{j}}{\partial_{y} u^{s}}=\frac{\partial_{y}^{2} u_{\theta_{j}}^{j}}{\partial_{y} u^{s}} \int_{0}^{y} w^{j}(t, x, \tilde{y}) d \tilde{y}+\frac{\partial_{y} u_{\theta_{j}}^{j}}{\partial_{y} u^{s}} w^{j}
$$


we have

$$
\begin{aligned}
\left\|\frac{\partial_{y} \delta u^{j}}{\partial_{y} u^{s}}\right\|_{\mathcal{A}_{\ell}^{k_{1}}} \leq & \left\|\frac{\partial_{y}^{2}\left(u_{\theta_{j}}^{j}-u^{s}\right)}{\partial_{y} u^{s}}\right\|_{\mathcal{A}_{\ell}^{k_{1}}}\left\|w^{j}\right\|_{\mathcal{C}_{\ell}^{0}}+\left\|\frac{\partial_{y}^{2} u^{s}}{\partial_{y} u^{s}}\right\|_{\mathcal{C}_{\ell}^{k_{1}}}\left\|w^{j}\right\|_{L_{\ell}^{2}} \\
& +\left\|\frac{\partial_{y}^{2} u_{\theta_{j}}^{j}}{\partial_{y} u^{s}}\right\|\left\|_{\mathcal{C}_{\ell}^{0}}\right\| w^{j}\left\|_{\mathcal{A}_{\ell}^{k_{1}}}+\right\| \frac{\partial_{y} u_{\theta_{j}^{j}}^{j}}{\partial_{y} u^{s}}\|\|_{L^{\infty}}\left\|w^{j}\right\|_{\mathcal{A}_{\ell}^{k_{1}}}+\left\|\frac{\partial_{y} u_{\theta_{j}}^{j}}{\partial_{y} u^{s}}\right\|_{\dot{\mathcal{A}}_{k_{1}}}\left\|w^{j}\right\|_{L_{\ell}^{\infty}} \\
\leq & C_{0} C_{11} \epsilon^{2} \theta_{j}^{\max \left\{3-\tilde{k}, k_{1}+5-2 \tilde{k}\right\}} \Delta \theta_{j}+\left(C_{11} \epsilon+C^{a}\right) C_{0} \epsilon \theta_{j}^{\max \left\{3-\tilde{k}, k_{1}-\tilde{k}\right\}} \Delta \theta_{j} \\
& +C^{a} C_{0} \epsilon \theta_{j}^{3-\tilde{k}} \Delta \theta_{j}+2 C_{0} \epsilon \theta_{j}^{\max \left\{3-\tilde{k}, k_{1}-\tilde{k}\right\}} \Delta \theta_{j} \\
& +\left(C_{11} \epsilon \theta_{j}^{\max \left\{0, k_{1}+1-\tilde{k}\right\}}+C^{a}\right) C_{0} \epsilon \theta_{j}^{3-\tilde{k}} \Delta \theta_{j},
\end{aligned}
$$

where we have used (7.9), (7.11), and (5.12).

If we choose a constant

$$
\tilde{C}_{11} \geq C_{0}\left(2+3 C^{a}+3 C_{11} \epsilon_{0}\right),
$$

then from (7.17), we get

$$
\left\|\frac{\partial_{y} \delta u^{j}}{\partial_{y} u^{s}}\right\|_{\mathcal{A}_{\ell}^{k_{1}}} \leq \tilde{C}_{11} \epsilon \theta_{j}^{\max \left\{3-\tilde{k}, k_{1}-\tilde{k}\right\}} \Delta \theta_{j},
$$

by using $\tilde{k} \geq 7$.

By using (7.18), Lemmas 5.7 and 5.8 , from (7.16), there exists a constant $C_{8}>0$ such that

$$
\left\|\frac{e_{j}^{(1)}}{\partial_{y} u^{s}}\right\|_{\mathcal{A}_{\ell}^{k_{1}}} \leq C_{8} \epsilon^{2} \theta_{j}^{\max \left\{6-2 \tilde{k}, k_{1}+3-2 \tilde{k}\right\}} \Delta \theta_{j},
$$

for all $k_{1} \leq k_{0}-1$.

Similarly,

$$
\begin{aligned}
\left\|\frac{e_{j}^{(2)}}{\partial_{y} u^{s}}\right\|_{\mathcal{A}_{\ell}^{k_{1}}} \leq & \left\|\left(\left(1-S_{\theta_{j}}\right)\left(u^{j}-u^{s}\right)\right) \frac{\delta u^{j}}{\partial_{y} u^{s}}\right\|_{\mathcal{A}_{\ell}^{k_{1}+1}} \\
& +\left\|\delta v^{j} \frac{\partial_{y}\left(\left(1-S_{\theta_{j}}\right)\left(u^{j}-u^{s}\right)\right)}{\partial_{y} u^{s}}\right\|_{\mathcal{A}_{\ell}^{k_{1}}}+\left\|\left(\left(1-S_{\theta_{j}}\right) v^{j}\right) \frac{\partial_{y}\left(\delta u^{j}\right)}{\partial_{y} u^{s}}\right\|_{\mathcal{A}_{\ell}^{k_{1}}},
\end{aligned}
$$


by using Lemma 2.1, the second formula of (4.1) and (5.21), we get

$$
\begin{aligned}
& \left\|\frac{e_{j}^{(2)}}{\partial_{y} u^{s}}\right\|_{\mathcal{A}_{\ell}^{k_{1}}} \leq M_{k_{1}}\left\{\left\|u^{j}-u^{s}\right\|_{\mathcal{A}_{\ell}^{k_{1}+1}}\left\|\frac{\delta u^{j}}{\partial_{y} u^{s}}\right\|_{L^{\infty}}\right. \\
& +\left\|\left(1-S_{\theta_{j}}\right)\left(u^{j}-u^{s}\right)\right\|_{L_{y, \ell}^{2}\left(L_{t, x}^{\infty}\right)}\left\|\frac{\delta u^{j}}{\partial_{y} u^{s}}\right\|_{\mathcal{D}_{0}^{k_{1}+1}} \\
& +\left\|\delta v^{j}\right\|_{L^{\infty}}\left\|\frac{\partial_{y}\left(u^{j}-u^{s}\right)}{\partial_{y} u^{s}}\right\|_{\mathcal{A}_{\ell}^{k_{1}}} \\
& +\left\|\delta v^{j}\right\|_{\mathcal{D}_{0}^{k_{1}}}\left\|\left(1-S_{\theta_{j}}\right)\left(\frac{\partial_{y}\left(u^{j}-u^{s}\right)}{\partial_{y} u^{s}}\right)\right\|_{L_{y, \ell}^{2}\left(L_{t, x}^{\infty}\right)} \\
& \left.+\left\|\left(1-S_{\theta_{j}}\right) v^{j}\right\|_{L^{\infty}}\left\|\frac{\partial_{y}\left(\delta u^{j}\right)}{\partial_{y} u^{s}}\right\|_{\mathcal{A}_{\ell}^{k_{1}}}+\left\|v^{j}\right\|_{\mathcal{D}_{0}^{k_{1}}}\left\|\frac{\partial_{y}\left(\delta u^{j}\right)}{\partial_{y} u^{s}}\right\|_{L_{y, \ell}^{2}\left(L_{t, x}^{\infty}\right)}\right\} \\
& \leq M_{k_{1}}\left\{\left\|u^{j}-u^{s}\right\|_{\mathcal{A}_{\ell}^{k_{1}+1}}\left\|\frac{\delta u^{j}}{\partial_{y} u^{s}}\right\|_{L^{\infty}}+\theta_{j}^{2-k^{\prime}}\left\|u^{j}-u^{s}\right\|_{\mathcal{A}_{\ell}^{k^{\prime}}}\left\|\frac{\delta u^{j}}{\partial_{y} u^{s}}\right\|_{\mathcal{D}_{0}^{k_{1}+1}}\right. \\
& +\left\|\delta v^{j}\right\|_{L^{\infty}}\left\|\frac{\partial_{y}\left(u^{j}-u^{s}\right)}{\partial_{y} u^{s}}\right\|_{\mathcal{A}_{\ell}^{k_{1}}}+\theta_{j}^{2-k^{\prime}}\left\|\delta v^{j}\right\|_{\mathcal{D}_{0}^{k_{1}}}\left\|\frac{\partial_{y}\left(u^{j}-u^{s}\right)}{\partial_{y} u^{s}}\right\|_{\mathcal{A}_{\ell}^{k^{\prime}}} \\
& \left.+\theta_{j}^{2-k^{\prime}}\left\|v^{j}\right\|_{\mathcal{D}_{0}^{k^{\prime}}}\left\|\frac{\partial_{y}\left(\delta u^{j}\right)}{\partial_{y} u^{s}}\right\|_{\mathcal{A}_{\ell}^{k_{1}}}+\left\|v^{j}\right\|_{\mathcal{D}_{0}^{k_{1}}}\left\|\frac{\partial_{y}\left(\delta u^{j}\right)}{\partial_{y} u^{s}}\right\|_{L_{y, \ell}^{2}\left(L_{t, x}^{\infty}\right)}\right\},
\end{aligned}
$$

for a fixed integer $2 \leq k^{\prime} \leq k_{0}-2$.

By applying Lemma 5.7, Lemma 5.8, and estimates (7.9) and (7.18) to the above inequality, and by setting $k^{\prime} \geq \tilde{k}-2$, it follows that for $k_{1} \leq k_{0}-1$,

$$
\left\|\frac{e_{j}^{(2)}}{\partial_{y} u^{s}}\right\|_{\mathcal{A}_{l}^{k_{1}}} \leq C_{8} \epsilon^{2} \theta_{j}^{\max \left(3-\tilde{k}, k_{1}+5-2 \tilde{k}\right)} \Delta \theta_{j},
$$

for a positive constant $C_{8}$. And this completes the proof of the lemma.

\section{ACKNOWLEDGEMENTS}

The research of the first author was supported in part by the Zhiyuan foundation, Shanghai Jiao Tong University, and the National Science Foundation of China No. 11171211. The research of the second author was supported in part by the National Science Foundation of China No. 10971134, No. 11031001, and No. 91230102. The research of the third author was supported partially by the Fundamental Research Funds for the Central Universities and the National Science Foundation of China No. 11171261. The last author's research was supported by the General Research Fund of Hong Kong, CityU No. 104511, and the Croucher Foundation. The authors would like to express their gratitude to Weinan E and Zhouping Xin for their valuable discussions. 


\section{REFERENCES}

[1] Serge Alinhac and Patrick Gérard, Pseudo-differential operators and the Nash-Moser theorem, Graduate Studies in Mathematics, vol. 82, American Mathematical Society, Providence, RI, 2007. Translated from the 1991 French original by Stephen S. Wilson. MR2304160 (2007m:35001)

[2] Russel. E. Caflisch and Marco Sammartino, Existence and singularities for the Prandtl boundary layer equations, Z. Angew. Math. Mech. 80 (2000), no. 11-12, 733-744, DOI 10.1002/15214001(200011)80:11/12〈733::AID-ZAMM733〉3.0.CO;2-L. Special issue on the occasion of the 125th anniversary of the birth of Ludwig Prandtl. MR 1801538 (2002b:76047)

[3] Jacques Chazarain and Alain Piriou, Introduction to the theory of linear partial differential equations, Studies in Mathematics and its Applications, vol. 14, North-Holland Publishing Co., Amsterdam-New York, 1982. Translated from the French. MR678605 (83j:35001)

[4] Weinan E, Boundary layer theory and the zero-viscosity limit of the Navier-Stokes equation, Acta Math. Sin. (Engl. Ser.) 16 (2000), no. 2, 207-218, DOI 10.1007/s101140000034. MR:1778702(2001d:76037)

[5] Weinan E and Bjorn Engquist, Blowup of solutions of the unsteady Prandtl's equation, Comm. Pure Appl. Math. 50 (1997), no. 12, 1287-1293, DOI 10.1002/(SICI)10970312(199712)50:12〈1287::AID-CPA4〉3.0.CO;2-4. MR 1476316 (99c:35196)

[6] David Gérard-Varet and Emmanuel Dormy, On the ill-posedness of the Prandtl equation, J. Amer. Math. Soc. 23 (2010), no. 2, 591-609, DOI 10.1090/S0894-0347-09-00652-3. MR2601044(2011f:35259)

[7] David Gérard-Varet and Toan Trong Nguyen, Remarks on the ill-posedness of the Prandtl equation, Asymptot. Anal. 77 (2012), no. 1-2, 71-88. MR2952715

[8] Emmanuel Grenier, On the nonlinear instability of Euler and Prandtl equations, Comm. Pure Appl. Math. 53 (2000), no. 9, 1067-1091, DOI 10.1002/1097-0312(200009)53:9〈1067::AIDCPA1)3.3.CO;2-H. MR1761409 (2001i:76056)

[9] Yan Guo and Toan Nguyen, A note on Prandtl boundary layers, Comm. Pure Appl. Math. 64 (2011), no. 10, 1416-1438, DOI 10.1002/cpa.20377. MR2849481 (2012m:35239)

[10] Richard S. Hamilton, The inverse function theorem of Nash and Moser, Bull. Amer. Math. Soc. (N.S.) 7 (1982), no. 1, 65-222, DOI 10.1090/S0273-0979-1982-15004-2. MR656198 (83j:58014)

[11] Lan Hong and John K. Hunter, Singularity formation and instability in the unsteady inviscid and viscous Prandtl equations, Commun. Math. Sci. 1 (2003), no. 2, 293-316. MR1980477 (2005k:76035)

[12] Lars Hörmander, The analysis of linear partial differential operators, Vol. I-IV, Springer Verlag, Berlin, 1985.

[13] Lars Hörmander, The boundary problems of physical geodesy, Arch. Ration. Mech. Anal. 62 (1976), no. 1, 1-52. MR0602181 (58 \#29202a)

[14] Maria Carmela Lombardo, Marco Cannone, and Marco Sammartino, Well-posedness of the boundary layer equations, SIAM J. Math. Anal. 35 (2003), no. 4, 987-1004 (electronic), DOI 10.1137/S0036141002412057. MR2049030(2005a:76137)

[15] Guy Métivier, Small viscosity and boundary layer methods, Modeling and Simulation in Science, Engineering and Technology, Birkhäuser Boston, Inc., Boston, MA, 2004. Theory, stability analysis, and applications. MR2151414 (2007b:35004)

[16] Jürgen Moser, A new technique for the construction of solutions of nonlinear differential equations, Proc. Natl. Acad. Sci. USA 47 (1961), 1824-1831. MR0132859(24 \#A2695)

[17] John Nash, The imbedding problem for Riemannian manifolds, Ann. of Math. (2) 63 (1956), 20-63. MR0075639 (17,782b)

[18] Olga A. Oleinik, The Prandtl system of equations in boundary layer theory, Soviet Math Dokl. 4 (1963), 583-586.

[19] Olga A. Oleinik and VN Samokhin, Mathematical models in boundary layer theory, Applied Mathematics and Mathematical Computation, vol. 15, Chapman \& Hall/CRC, Boca Raton, FL, 1999. MR 1697762 (2000c:76021)

[20] Ludwig Prandtl, Uber Flüssigkeitsbewegungen bei sehr kleiner Reibung. In "Verh. Int. Math. Kongr., Heidelberg 1904," Teubner, 1905. 
[21] Marco Sammartino and Russel E. Caflisch, Zero viscosity limit for analytic solutions of the Navier-Stokes equation on a half-space. II. Construction of the Navier-Stokes solution, Comm. Math. Phys. 192 (1998), no. 2, 463-491, DOI 10.1007/s002200050305. MR 1617538 (99d:35129b)

[22] Zhouping Xin and Liqun Zhang, On the global existence of solutions to the Prandtl's system, Adv. Math. 181 (2004), no. 1, 88-133, DOI 10.1016/S0001-8708(03)00046-X. MR2020656 (2005f:35219)

Department of Mathematics, Shanghai Jiao Tong University, Shanghai, 200240, P. R. China and Arts et Métiers Paristech, Paris 75013, France

E-mail address: radjesvarane.alexandre@paristech.fr

Department of Mathematics, and MOE-LSC, Shanghai Jiao Tong University, ShangHAI, 200240, P. R. CHINA

E-mail address: ygwang@sjtu.edu.cn

School of Mathematics, Wuhan University 430072, Wuhan, P. R. China, and UniverSité de Rouen, UMR 6085-CNRS, Mathématiques, Avenue de l’Université, BP.12, 76801 Saint Etienne du Rouvray, France

E-mail address: Chao-Jiang.Xu@univ-rouen.fr

Department of Mathematics, City University of Hong Kong, Hong Kong, P. R. China

E-mail address: matyang@cityu.edu.hk 